\title{
Old open clusters as key tracers of Galactic chemical evolution
}

\section{Fe abundances in NGC 2660, NGC 3960, and Berkeley $32^{\star, \star \star}$}

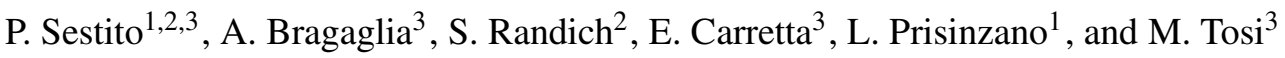 \\ 1 INAF - Osservatorio Astronomico "G.S. Vaiana" di Palermo, Piazza del Parlamento 1, 90134 Palermo, Italy \\ e-mail: sestito@arcetri.astro.it \\ 2 INAF - Osservatorio Astrofisico di Arcetri, Largo E. Fermi 5, 50125 Firenze, Italy \\ 3 INAF - Osservatorio Astronomico di Bologna, via C. Ranzani 1, 40127 Bologna, Italy
}

Received 9 March 2006 / Accepted 14 June 2006

\section{ABSTRACT}

\begin{abstract}
Aims. We obtained high-resolution UVES/FLAMES observations of a sample of nine old open clusters spanning a wide range of ages and Galactocentric radii. The goal of the project is to investigate the radial metallicity gradient in the disk, as well as the abundance of key elements ( $\alpha$ and Fe-peak elements). In this paper we present the results for the metallicity of three clusters: NGC 2660 (age $\sim 1 \mathrm{Gyr}$, Galactocentric distance of $8.68 \mathrm{kpc})$, NGC 3960 ( 1 Gyr, $7.80 \mathrm{kpc})$, and Be 32 ( 6-7 Gyr, $11.30 \mathrm{kpc})$. For Be 32 and NGC 2660, our study provides the first metallicity determination based on high-resolution spectra.

Methods. We performed equivalent width analysis with the spectral code MOOG, which allows us to define a metallicity scale and build a homogeneous sample.

Results. We find that NGC 3960 and NGC 2660 have a metallicity that is very close to solar $([\mathrm{Fe} / \mathrm{H}]=+0.02$ and +0.04 , respectively), while the older $\mathrm{Be} 32$ turns out to have $[\mathrm{Fe} / \mathrm{H}]=-0.29$.
\end{abstract}

Key words. stars: abundances - stars: evolution - Galaxy: disk - open clusters and associations: individual: NGC 2660 open clusters and associations: individual: NGC 3960 - open clusters and associations: individual: Berkeley 32

\section{Introduction}

The investigation of chemical abundances in stars is fundamental for comprehending the Galaxy formation mechanisms and the subsequent evolution. In recent years, several observational and theoretical studies have been carried out, aimed at understanding various features, such as the star formation history in the disk and its Galactocentric distribution, the gas distribution and stellar density, the metallicity distribution $([\mathrm{Fe} / \mathrm{H}])$ in the disk, as well as the abundances of other elements relative to $\mathrm{Fe}$ (e.g. Edvardsson et al. 1993; Friel et al. 2003; Carretta et al. 2004, 2005; Yong et al. 2005). Although many observational characteristics of the Milky Way are reproduced well by the appropriate theoretical models (Lacey \& Fall 1985; Tosi 1988; Giovagnoli \& Tosi 1995; Chiappini et al. 1997; Boissier \& Prantzos 1999), several problems persist.

One of these problems concerns the radial metallicity gradient in the disk, i.e. the distribution of chemical elements with Galactocentric distance $\left(R_{\mathrm{gc}}\right)$ and its evolution with age. The radial metallicity gradient and its temporal evolution are among the most critical constraints on Galactic chemical evolution models, since predictions of its slope and slope variations with time depend mainly on the relative timescales of the gas consumption (i.e. star formation rate) and gas accretion (i.e. infall rate), that is with the disk formation scenario. At the same

^ Based on observations collected at ESO telescopes under programs 072.D-0550 and 074.D-0571.

$\star \star$ Tables 7, 9-11 are only available in electronic form at http://www . aanda. org time, the abundances of $\alpha$ and Fe-peak elements and their ratios to $\mathrm{Fe}$ are crucial for getting insight into the role of stars with different masses and evolutionary lifetimes in the heavy element enrichment of the interstellar medium.

The distribution of heavy elements with Galactocentric distance is often investigated through observations of objects like H II regions and B stars (e.g. Shaver et al. 1983; Smartt \& Rollerstone 1997), which are bright and measurable also in external galaxies. They show a negative gradient, but it is not clear if the slope changes with position in the disk and, given the young ages of all these objects, it is impossible to evaluate whether it varies with age. Good indicators are also planetary nebulæ (PNe); observations of type II PNe (whose progenitors are $\sim 2-3$ Gyr old) reveal the presence of a gradient similar to that traced by $\mathrm{H}$ II regions, but its precise slope is still under debate (e.g. Pasquali \& Perinotto 1993; Maciel et al. 2003), since the investigation of PNe is affected by the uncertainty on their distances. In our opinion, one of the best tools for the investigation of radial abundance distributions is represented by open clusters, since they cover a wide range of ages, metallicities, and positions in the Galactic disk.

Various observational studies have already addressed the problem of the metallicity distribution through open cluster observations, but the general picture has not been delineated well yet, and discrepant results have been obtained by different authors. For example, Friel (1995, 2006), Carraro et al. (1998) and Friel et al. (2002) suggest the presence of a negative $[\mathrm{Fe} / \mathrm{H}]$ gradient, while Twarog et al. (1997) and Corder \& Twarog (2001) favor a step-like distribution of the Fe content 
Table 1. Target clusters and their properties. Literature sources are reported in the text.

\begin{tabular}{llllll}
\hline \hline Cluster & $\begin{array}{l}\text { Age } \\
(\mathrm{Gyr})\end{array}$ & {$[\mathrm{Fe} / \mathrm{H}]$} & $\begin{array}{l}R_{\mathrm{gc}} \\
(\mathrm{kpc})\end{array}$ & $\begin{array}{l}(m-M)_{0} \\
(\mathrm{mag})\end{array}$ & $\begin{array}{l}E(B-V) \\
(\mathrm{mag})\end{array}$ \\
\hline NGC 3960 & $0.9 \rightarrow 1.4$ & $-0.68 \rightarrow-0.06$ & 7.80 & 11.60 & 0.29 (differential) \\
NGC 2660 & $\sim 1$ & solar/sub-solar & 8.68 & 12.20 & 0.40 \\
Be 32 & $\sim 6 \rightarrow 7$ & $-0.50 \rightarrow-0.37$ & 11.30 & 12.48 & 0.10 \\
\hline
\end{tabular}

with Galactocentric distance. Furthermore, recent results (e.g., Yong et al. 2005) suggest that a single slope is not a good fit to the data when clusters farther than about $14 \mathrm{kpc}$ from the center are included. Even when restricted to metallicities derived by spectroscopic data, large differences appear to be present among different analyses.

The discrepancies among the various literature results can be ascribed to several concurring factors: the number of stars employed (statistics), the quality of the spectra (signal-to-noise $S / N$ - and spectral resolution) and, above all, the method of analysis, including continuum tracing, atomic parameters, equivalent width $(E W)$ measurement, model atmospheres, spectral code, and atmospheric parameters. Diverse methods of analysis and physical assumptions are adopted by different authors, so that the resulting chemical abundances have been often referred to discrepant scales of temperature and metallicity. An investigation of this kind instead needs to rely on a large sample of open clusters for which element abundances (but also distances and ages) are derived with the same method in order to avoid spurious results due to an inhomogeneous analysis.

Although the number of open clusters studied with highresolution spectroscopy is steadily increasing, we still lack a suitable sample for deriving truly reliable constraints on the gradient and its evolution with time. To this aim and in the context of a VLT/FLAMES program on Galactic open clusters (Randich et al. 2005), we collected UVES spectra of evolved stars in a variety of old open clusters. Our sample includes To 2 ( 2-3 Gyr), NGC 6253 ( $\sim 3$ Gyr), Be 29 ( 4 Gyr), Be 20 ( $\sim$ Gyr), Be 32 ( 6-7 Gyr) and NGC 2324, NGC 2477, NGC 2660, NGC 3960 (ages $\lesssim 1 \mathrm{Gyr}$ ). Likewise $R_{\mathrm{gc}}$ varies between $\sim 7$ and $21 \mathrm{kpc}$ (Be 29 being the most distant cluster found so far). The primary goal of the UVES observations is to determinate the metallicity of the sample clusters using a homogeneous method of analysis: in this way, we will have a new rather large sample with all the clusters on the same abundance scale. At the same time we derive - in most cases for the first time - abundances of other key elements, as $\mathrm{CNO}, \alpha$, and Fe-peak elements, as well as sand r-process elements, which are fundamental for understanding Galactic formation and evolution.

The emphasis of this paper is on presenting the method of analysis for $[\mathrm{Fe} / \mathrm{H}]$ and on determining the metallicity of three of the open clusters included in the project (NGC 3960, NGC 2660, Be 32). The analysis of $\alpha$ elements and Fe-peak elements in the same clusters and in NGC 2477, NGC 2324 is deferred to a forthcoming paper (Bragaglia et al. 2006b, in preparation). NGC 3960 was recently investigated through high-resolution spectroscopy by Bragaglia et al. (2006a, hereafter B06a), therefore it is well-suited for a direct comparison with another spectroscopic analysis and to estimate possible sources of discrepancies between different studies at high resolution. As far as the other clusters are concerned, neither NGC 2660 nor Be 32 have ever been studied using high-resolution spectroscopy.

The paper is organized as follows: in Sect. 2 we present the properties of the target clusters and in Sect. 3 we describe observations and data reduction. In Sect. 4 we give a detailed description of the method of analysis and inputs adopted (e.g., line lists and atomic parameters). The results for $\mathrm{Fe}$ abundances in the three open clusters are reported in Sect. 5 and discussed in Sect. 6. A summary closes this paper (Sect. 7).

\section{Target clusters}

The main properties of the three open clusters investigated in this paper are reported in Table 1.

NGC 3960 is located at a Galactocentric distance $R_{\mathrm{gc}}=$ $7.8 \mathrm{kpc}$. The first CMD for the cluster was published by Janes (1981), based on $B V$ photographic data. More recently, Prisinzano et al. (2004) investigated this cluster by collecting data in the $B V I$ bands with the Wide Field Imager at the ESOMax Planck $2.2 \mathrm{~m}$ telescope. These authors found strong indications of differential reddening in the direction of the cluster, with $E(B-V)$ varying from 0.16 to 0.62 over their $\approx 30 \times 30 \mathrm{arcmin}^{2}$ field of view. They found $E(B-V)=0.29$ towards the cluster center (in agreement with Janes 1981), $(m-M)_{0}=11.35$, and age ranging from 0.9 to $1.4 \mathrm{Gyr}$. The most recent work on NGC 3960 is the photometric and spectroscopic study by B06a, who find $(m-M)_{0}=11.60$ and an average $E(B-V)=$ $0.29 \pm 0.02$ (over a field of view of $13.3 \times 13.3 \mathrm{arcmin}^{2}$ ), with differential reddening of $\pm 0.05 \mathrm{mag}$ (in agreement with the values inferred by Prisinzano et al. in their central region, corresponding to B06a field of view) and a slightly sub-solar metallicity, $[\mathrm{Fe} / \mathrm{H}]=-0.12$. This is the only metallicity estimate based on high-resolution spectroscopy. Previous reports suggested a lower Fe content: for example, Friel \& Janes (1993) quoted $[\mathrm{Fe} / \mathrm{H}]=-0.34$ from low-resolution spectroscopy. Other estimates range from $[\mathrm{Fe} / \mathrm{H}]=-0.68$ (Geisler et al. 1992; from Washington photometry) to -0.06 (Piatti et al. 1995, using a DDO abundance calibration). NGC 3960 has been included in several other studies; for example, by Twarog et al. (1997), who determined a metallicity of -0.17 , based on a homogenization of DDO photometry and low-resolution spectroscopy, and by Mermilliod et al. (2001), who determined precise radial velocities for a number of red giants.

NGC 2660 was first investigated by Hartwick \& Hesser (1973; photographic and photoelectric study), and a more recent analysis was performed by Sandrelli et al. (1999), who presented CCD UBVI photometry and estimated an age of $\sim 1 \mathrm{Gyr}$ or slightly less, a distance modulus $(m-M)_{0}=12.20$, and reddening $E(B-V) \sim 0.40$, implying a Galactocentric distance of $8.68 \mathrm{kpc}$ (Bragaglia \& Tosi 2006). The cluster metallicity has been investigated by various authors but only on the basis of photometry, and with inconsistent results: Sandrelli et al. (1999) quoted a nearly solar Fe content (from stellar evolutionary tracks), while previous works reported a sub-solar $[\mathrm{Fe} / \mathrm{H}]$ (e.g., Hesser \& Smith 1987; Piatti et al. 1995, both from DDO photometry).

As pointed out in Sect. 1, the main goal of this project is to investigate the radial metallicity gradient in the disk and its temporal evolution. Be 32 is $\sim 6-7$ Gyr old, which is relevant for the evolution of the gradient with age. Although the most 
Table 2. Observation log of NGC 3960. One star is common to configurations A and B; we consider only clump objects here (7 stars in total). The remaining fibers were assigned to sky and to MS stars.

\begin{tabular}{llllll}
\hline \hline Date & UT $_{\text {beginning }}$ & $\begin{array}{l}\text { Exposure time } \\
\text { (s) }\end{array}$ & Configuration & Grating & $\begin{array}{l}\text { No. of stars } \\
\text { (clump) }\end{array}$ \\
\hline $2004-04-03$ & 025739.980 & 2595 & $\mathrm{~A}$ & $\mathrm{CD} 4$ & 4 \\
$2004-04-03$ & 035216.186 & 2595 & $\mathrm{~A}$ & $\mathrm{CD} 4$ & 4 \\
$2004-04-03$ & 044731.818 & 2595 & $\mathrm{~A}$ & $\mathrm{CD} 3$ & 4 \\
$2004-04-20$ & 033739.635 & 2595 & $\mathrm{~B}$ & $\mathrm{CD} 3$ & 4 \\
$2004-05-02$ & 001231.740 & 2595 & $\mathrm{~B}$ & $\mathrm{CD} 4$ & 4 \\
$2004-05-02$ & 005737.007 & 2595 & $\mathrm{~B}$ & $\mathrm{CD} 4$ & 4 \\
$2004-05-02$ & 014313.805 & 2595 & $\mathrm{~B}$ & $\mathrm{CD} 3$ & 4 \\
$2004-05-02$ & 023918.200 & 2595 & $\mathrm{~A}$ & $\mathrm{CD} 3$ & 4 \\
\hline
\end{tabular}

Table 3. Data for NGC 3960. $\mathrm{ID}_{\text {phot }}$ and BVI photometry (non corrected for differential reddening) are from Prisinzano et al. (2004). The number of exposures for each star is intended as the number of pointings with the same cross-disperser.

\begin{tabular}{|c|c|c|c|c|c|c|c|c|c|c|c|c|}
\hline $\begin{array}{l}\text { Star } \\
\text { ID }_{\text {fla }}\end{array}$ & $\begin{array}{l}\text { Star } \\
\text { ID }_{\text {phot }}\end{array}$ & RA & Dec & $B$ & $\bar{V}$ & $I$ & $J_{2 \text { MASS }}$ & $K_{2 \mathrm{MASS}}$ & No. exp. & $\begin{array}{l}R V \\
\left(\mathrm{~km} \mathrm{~s}^{-1}\right)\end{array}$ & $S / N$ & Notes \\
\hline $\mathrm{c} 1$ & 310753 & 115006.330 & -554413.00 & 15.866 & 14.262 & 12.496 & 11.213 & 10.238 & 2 & +1.83 & $60-80$ & NM \\
\hline c3 & 310755 & 115033.149 & -554236.13 & 14.799 & 13.514 & 12.088 & 11.079 & 10.357 & 2 & -24.16 & $120-130$ & M \\
\hline c4 & 310756 & 115028.184 & -554136.66 & 14.366 & 13.194 & 11.907 & 10.989 & 10.304 & 2 & -22.39 & $95-115$ & $\mathrm{M}$ \\
\hline$c 5$ & 310757 & 115036.050 & -554205.40 & 14.323 & 13.062 & 11.679 & 10.671 & 9.959 & 2 & -21.94 & $110-130$ & M \\
\hline c6 & 310758 & 115026.750 & -554028.20 & 14.084 & 12.945 & 11.697 & 10.815 & 10.133 & 4 & -21.86 & $115-160$ & M \\
\hline $\mathrm{c} 8$ & 310760 & 115037.621 & -554015.19 & 14.224 & 13.060 & 11.758 & 10.820 & 10.162 & 2 & -32.87 & $120-190$ & bin, $\mathrm{M}$ \\
\hline c9 & 310761 & 115038.100 & -553944.50 & 14.309 & 13.100 & 11.787 & 10.839 & 10.151 & 2 & -22.58 & $100-110$ & M \\
\hline
\end{tabular}

distant cluster in our sample is Be $29\left(R_{\mathrm{gc}} \sim 21 \mathrm{kpc}\right), \mathrm{Be} 32$ is the most distant among the three clusters analyzed in this paper and, with a Galactocentric radius $>10 \mathrm{kpc}$, it is located beyond the $[\mathrm{Fe} / \mathrm{H}]$ vs. $R_{\mathrm{gc}}$ discontinuity found by Twarog et al. (1997). Therefore, Be 32 is the most interesting of the three clusters. Photometric studies of the cluster were carried out by Kaluzny \& Mazur (1991), Richtler \& Sagar (2001), Hasegawa et al. (2004) and D'Orazi et al. (2006). The last study finds reddening and distance modulus $E(B-V)=0.10$ and $(m-M)_{0}=12.48$. As for the metal content, a sub-solar Fe has been suggested for the cluster, e.g., by Noriega-Mendoza \& Ruelas-Mayorga (1997), who found $[\mathrm{Fe} / \mathrm{H}]=-0.37$ based on the CMD, by Friel et al. (2002) based on low-resolution spectroscopy $([\mathrm{Fe} / \mathrm{H}]=-0.50)$, and by D'Orazi et al. (2006) based on evolutionary tracks $(Z=0.008)$.

\section{Observations and data reduction}

The open clusters included in the program were all observed with the multi-object instrument FLAMES on VLT/UT2 (ESO, Chile; Pasquini et al. 2000). The fiber link to UVES was used to obtain high-resolution spectra $(R=40000)$ for red giant branch (RGB) and clump objects.

NGC 3960 was observed in Service mode in 2004; two FLAMES configurations were used, and UVES observations were performed for both configurations with two different gratings (CD3 and $\mathrm{CD} 4$, covering the wavelength ranges 4750-6800 $\AA$ and 6600-10600 $\AA$, respectively). A log of observations (date, UT, exposure time, grating, configuration, number of stars) is given in Table 2. The spectra were reduced by ESO personnel using the dedicated pipeline, and we analyzed the $1-d$, wavelength-calibrated spectra using standard IRAF ${ }^{1}$ packages.

${ }^{1}$ IRAF is distributed by the National Optical Astronomical Observatories, which are operated by the Association of Universities for Research in Astronomy, under contract with the National Science Foundation.
Table 3 provides information on the target stars. We observed 7 red clump and 3 main sequence (MS) stars, but we only present here the results for the giants. We adopt hereafter the provisional identification number used for FLAMES pointing (Col. 1); however, since BVI photometry was taken from Prisinzano et al. (2004), we also show in Col. 2 the ID from their catalogue. $J$ and $K$ magnitudes come from the Two Micron All Sky Survey (2MASS ${ }^{2}$, Cutri et al. 2003). The MS stars were eventually discarded since they are rather faint, too warm (late A or early F spectral type), and rotate too rapidly. They are also not suitable for a detailed chemical abundance analysis, since the $S / N$ is too low and their lines are broad and shallow.

Radial velocities were measured using RVIDLINES on several tens of metallic lines on the individual spectra, then multiple spectra were combined. One fiber for configuration was used to register the sky value, but the correction was negligible. We corrected the spectra for the contamination by atmospheric telluric lines using TELLURIC in IRAF and an early-type star observed with UVES for another program. The $R V \mathrm{~s}$ for each star (shown in Table 3) have an attached uncertainty of less than about $0.5 \mathrm{~km} \mathrm{~s}^{-1}$, as deduced from the rms when averaging values obtained from different exposures; this error is also valid for the two other clusters. We derived an average heliocentric $R V$ of $-22.6 \pm 0.9$ (statistical error) $\mathrm{km} \mathrm{s}^{-1}$ for NGC 3960. Star c1 turned out to be a non-member on the basis of the radial velocity and spectral characteristics and is disregarded from now on (see also Mermilliod et al. 2001). Star c8 is a long-period spectroscopic binary (star 50 in Mermilliod et al. 2001), so its radial velocity appears slightly discrepant with respect to the cluster average; nevertheless it is compatible with it, once the amplitude of the $R V$ curve is considered. The spectral features and

2 The Two Micron All Sky Survey is a joint project of the University of Massachusetts and the Infrared Processing and Analysis Center/California Institute of Technology, funded by the national Aeronautics and Space Administration and the National Science Foundation. 
Table 4. Observation log of Be 32 and NGC 2660. One star is common to configurations A and B in Be 32 (10 stars in total). The remaining fibers were assigned to the sky.

\begin{tabular}{lllllll}
\hline \hline Cluster & Date & UT $_{\text {beginning }}$ & $\begin{array}{l}\text { Exp. time } \\
\text { (s) }\end{array}$ & Config. & Grating & No. of stars \\
\hline Be 32 & $2005-01-20$ & 004700 & 3600 & A & CD3 & 7 \\
Be 32 & $2005-01-20$ & 020153 & 3600 & A & CD4 & 7 \\
Be 32 & $2005-01-20$ & 031103 & 3600 & A & CD4 & 7 \\
Be 32 & $2005-01-20$ & 042341 & 3600 & A & CD3 & 7 \\
Be 32 & $2005-01-21$ & 004526 & 3600 & B & CD3 & 4 \\
NGC 2660 & $2005-01-23$ & 080202 & 3600 & - & CD3 & 5 \\
\hline
\end{tabular}

Table 5. Data for Be 32. $\mathrm{ID}_{\text {phot }}$, and $B V I$ are from D'Orazi et al. (2006), while $\mathrm{ID}_{\mathrm{fla}}$ is the identification number used for FLAMES observations.

\begin{tabular}{|c|c|c|c|c|c|c|c|c|c|c|c|c|}
\hline $\begin{array}{l}\text { Star } \\
\text { ID }_{\text {fla }}\end{array}$ & $\begin{array}{l}\text { Star } \\
\text { ID }_{\text {phot }}\end{array}$ & RA & Dec & $B$ & $V$ & $I$ & $J_{2 \text { MASS }}$ & $K_{2 \mathrm{MASS}}$ & No. exp. & $\begin{array}{l}R V \\
\left(\mathrm{~km} \mathrm{~s}^{-1}\right)\end{array}$ & $S / N$ & Notes \\
\hline 17 & 533 & 6588.242 & 62419.48 & 14.754 & 13.667 & 12.540 & 11.683 & 11.028 & 2 & 105.3 & $80-100$ & $\mathrm{M}$ \\
\hline 18 & 997 & 65813.762 & 62754.89 & 14.730 & 13.661 & 12.534 & 11.709 & 11.069 & 2 & 105.5 & 80-100 & M \\
\hline 19 & 787 & 6583.089 & 62616.08 & 14.800 & 13.709 & 12.564 & 11.722 & 11.024 & 2 & 101.4 & 70-90 & M \\
\hline 25 & 121 & 65759.819 & 62659.93 & 15.387 & 14.242 & 13.028 & 12.153 & 11.450 & 1 & 105.5 & $45-60$ & M \\
\hline 27 & 605 & 6582.262 & 62456.71 & 15.511 & 14.372 & 13.177 & 12.282 & 11.607 & 2 & 105.6 & $60-70$ & M \\
\hline 45 & 1139 & 6587.473 & 62932.67 & 16.299 & 15.278 & 14.153 & 13.341 & 12.707 & 3 & 105.3 & $45-50$ & M \\
\hline 932 & 1183 & 6581.771 & 62955.32 & 14.450 & 13.425 & 12.282 & 11.474 & 10.843 & 2 & 30.8 & 90-100 & NM \\
\hline 938 & - & 65811.488 & 62116.25 & - & $13.672 *$ & $12.501 *$ & 11.686 & 11.027 & 1 & 106.1 & $50-60$ & M \\
\hline 940 & 104 & 65822.909 & 62625.26 & 14.793 & 13.691 & 12.535 & 11.691 & 11.023 & 1 & 105.0 & $70-75$ & M \\
\hline 941 & 99 & 65750.572 & 62611.92 & 14.769 & 13.663 & 12.477 & 11.643 & 10.973 & 2 & 105.5 & $85-95$ & M \\
\hline
\end{tabular}

* $V$ and $I$ from Kaluzny \& Mazur (1991).

Table 6. Data for NGC 2660. ID and BVI photometry are from Sandrelli et al. (1999).

\begin{tabular}{lllllllllll}
\hline \hline Star & RA & Dec & $B$ & $V$ & $I$ & $J_{2 \text { MASS }}$ & $K_{2 \text { MASS }}$ & $\begin{array}{l}R V \\
\left(\mathrm{~km} \mathrm{~s}^{-1}\right)\end{array}$ & $S / N$ & Notes \\
\hline 296 & 84236.411 & -471207.26 & 15.798 & 14.552 & 13.174 & 11.680 & 10.636 & 21.73 & $45-65$ & $\mathrm{M}$ \\
318 & 84236.693 & -471037.06 & 15.401 & 14.110 & 12.713 & 11.586 & 10.828 & 20.66 & $50-65$ & $\mathrm{M}$ \\
542 & 84241.704 & -471125.22 & 15.420 & 14.120 & 12.704 & 11.603 & 10.837 & 20.47 & $45-90$ & $\mathrm{M}$ \\
694 & 84245.418 & -471118.71 & 15.674 & 14.368 & 12.938 & 11.791 & 11.049 & 21.47 & $45-55$ & $\mathrm{M}$ \\
862 & 84250.651 & -471303.61 & 15.602 & 14.315 & 12.909 & 11.770 & 11.046 & 21.47 & $50-70$ & $\mathrm{M}$ \\
\hline
\end{tabular}

average metallicity (see Sect. 5) clearly indicate that c8 is a cluster member.

Be 32 and NGC 2660 were observed in Visitor mode in 2005 January 19-22, and a log of the observations is given in Table 4. Be 32 was targeted using two FLAMES configurations, For configuration $\mathrm{A}$, the observations were carried out with both CD3 and CD4 gratings, while only CD3 was employed for configuration B For NGC 2660, we got only one exposure with the CD3 grating. The spectra were reduced by us, using the dedicated UVES pipeline (Mulas et al. 2002). Since observations were performed at full Moon and in the presence of cirrus, the background signal is high; nevertheless, we were able to carry out a good background subtraction using, as is customary, the fibers dedicated to the sky. Information on radial velocities and photometry are recorded in Tables 5 and 6 for Be 32 (10 stars) and NGC 2660 (5 stars), respectively.

In Table 5 we report the provisional ID for the stars used for the FLAMES pointing (adopted in the following) and those from the photometry by D'Orazi et al. (2006). Note that for star 938 the photometry was retrieved from Kaluzny \& Mazur (1991) and that star 932 turned out to be a non-member, because of both its $R V$ and its spectral features; therefore it has been dropped from the analysis. Identification numbers and $B V I$ magnitudes for NGC 2660 (listed in Table 6) were taken from Sandrelli et al. (1999).
The average heliocentric $R V \mathrm{~s}$ for Be 32 and NGC 2660 are $\langle R V\rangle=+106.0 \pm 1.4 \mathrm{~km} \mathrm{~s}^{-1}$ and $\langle R V\rangle=+21.2 \pm 0.6 \mathrm{~km} \mathrm{~s}^{-1}$, respectively. The $S / N$ given in Tables 3, 5, 6 refers to the spectra centered at $5800 \AA$, on which our analysis rests, measured using the task SPLOT within IRAF. Considering the wavelength regions around $\sim 5600$ and $6300 \AA$, the final combined spectra of NGC 3960 have typical $S / N \sim 100-160$; lower values result for Be 32, which has $S / N \sim 50-100$. Finally, spectra of NGC 2660, for which only one exposure for each star was available, have typical $S / N$ of $\sim 45-70$.

Figure 1 shows the CMDs for the three open clusters in the $(B-V, V)$ plane for NGC 3960 and NGC 2660, while for Be 32 we considered the $(V-I)$ colors because the $B$ magnitude of star 938 is not available. Circles (members) and squares (nonmembers) mark the stars observed with UVES.

We show the spectra of all the clump stars in the sample (cluster members) in Figs. 2-4. For clarity we restricted the plot to a region of $\sim 100 \AA$ at $6500-6600 \AA$.

\section{Abundance analysis}

The analysis of chemical abundances was carried out with an updated version (2005) of the spectral program MOOG (Sneden 1973) ${ }^{3}$ and using model atmospheres by Kurucz (1993). Like all

\footnotetext{
${ }^{3}$ http://verdi.as.utexas.edu/
} 

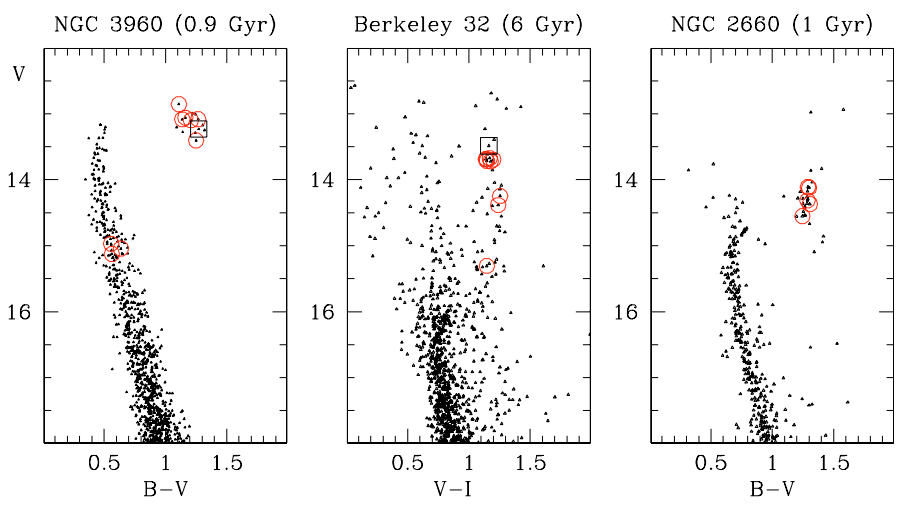

Fig. 1. Color-magnitude diagrams for the three clusters. From left to right: NGC 3960, Be 32, and NGC 2660. Note that the first and the last clusters are reported in the $(B-V, V)$ plane, while Be 32 is in the $(V-I$, $V)$ one. The observed stars are shown by circles (members) and squares (non-members).

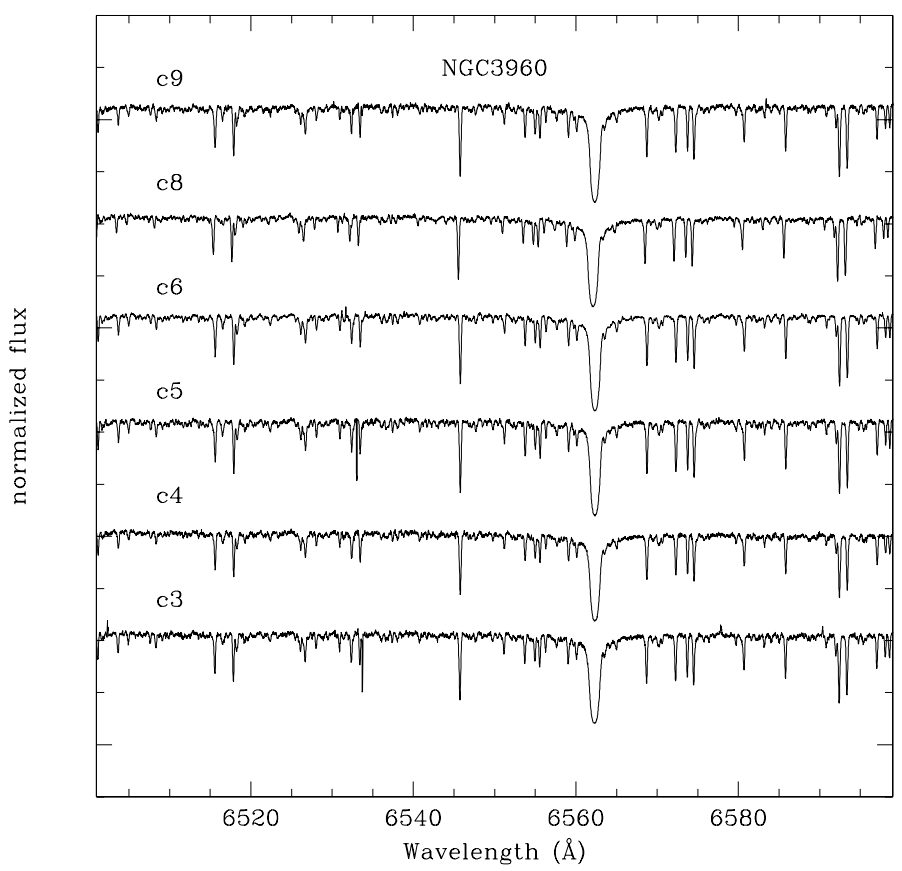

Fig. 2. NGC 3960 sample spectra in the spectral region at 6500-6600 $\AA$.

the commonly used spectral analysis codes, MOOG performs a local thermodynamic equilibrium (LTE) analysis.

\subsection{Solar analysis}

The first step is the determination of the solar Fe abundance, which allows us to fix a zero-point for the metallicity scale. This differential analysis minimizes errors in the results, especially for stars with nearly solar metallicity.

The line list adopted for the Sun is the one used by Gratton et al. (2003, hereafter G03), which includes 180 and 40 features for $\mathrm{Fe}$ I and Fe II, respectively, in the wavelength range $\sim 4100-6800 \AA$. Either theoretical or laboratory oscillator strengths $(\log g f)$ were considered, retrieved from the works by the Oxford group (e.g. Blackwell et al. 1986), and from Bard et al. (1991), O'Brian et al. (1991), and Bard \& Kock (1994). Most of the equivalent widths adopted for the Sun are from Rutten \& Van der Zalm (1984), who performed measurements

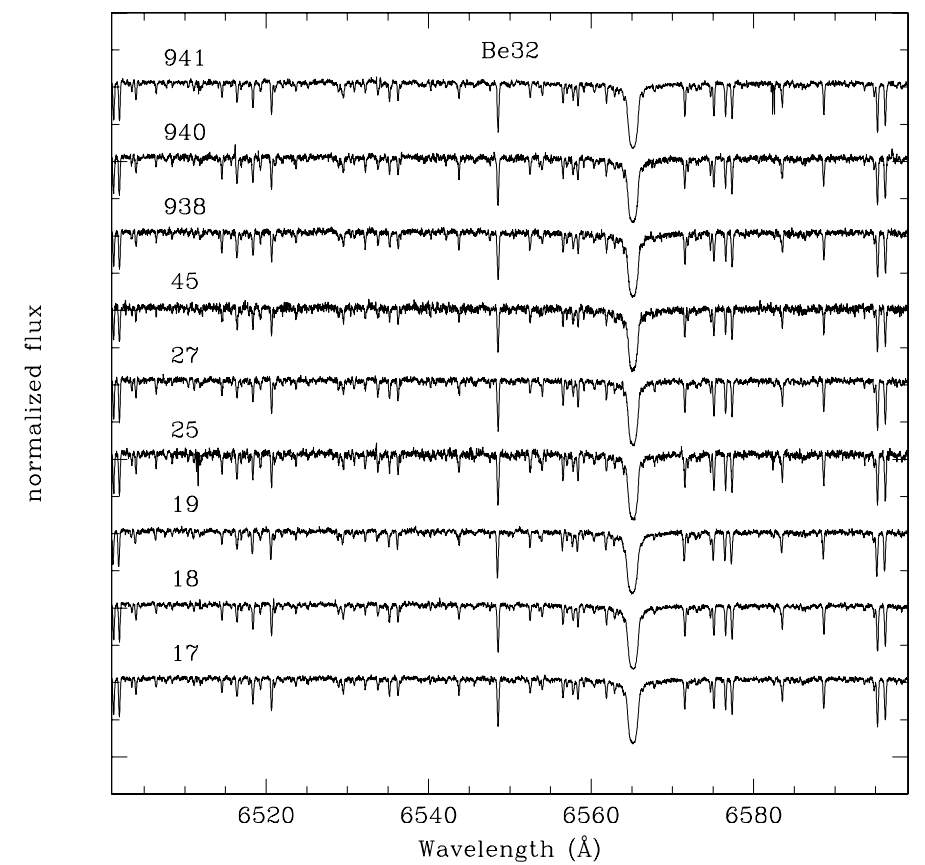

Fig. 3. Be 32 sample spectra.

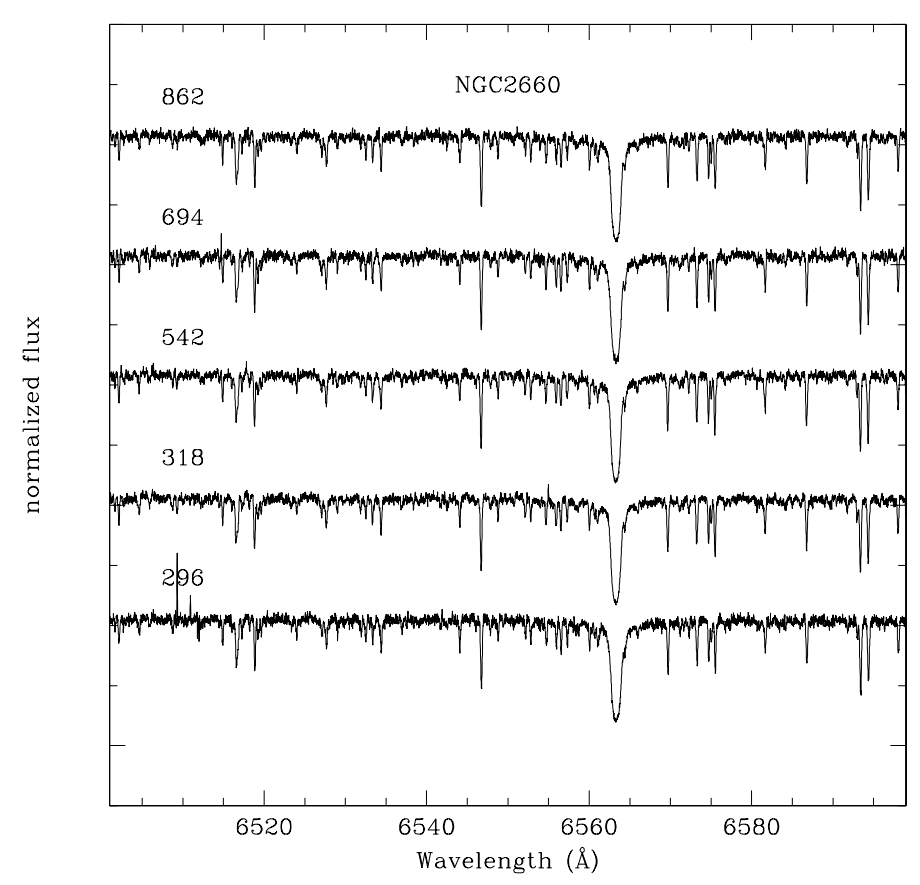

Fig. 4. NGC 2660 sample spectra.

on the Sacramento Peak Irradiance Atlas (Beckers et al. 1976); this set was integrated with measurements performed by G03 on the solar spectrum atlases by Delbouille et al. (1973) and by Kurucz (1984). Note that the solar spectra employed have much higher resolution than our UVES spectra.

When available, we adopted collisional damping coefficients from Barklem et al. (2000), who provide the best theoretical models for most transitions ${ }^{4}$. When the coefficients by Barklem et al. were not available we considered classical damping constants $\left(C_{6}\right)$ computed with the approximation of Unsöld (1955)

\footnotetext{
${ }^{4}$ MOOG was recently updated in order to give the possibility of using the Barklem coefficients.
} 
and multiplied by an enhancement factor $E$, given by: $\log E=$ $(0.381 \pm 0.017) E P-(0.88 \pm 0.33)$ where $E P$ is the excitation potential. This expression was obtained by G03 from several hundred Fe I features with available accurate collisional damping parameters and for $T_{\text {eff }}=5000 \mathrm{~K}$, typical of giant stars.

The line list for the Sun is available in electronic form (Table 7); the table includes wavelengths, name of the element (Fe I or Fe II), EP, $\log g f, E W$, and references for the damping coefficients. We computed the Fe content $\left(\log n(\mathrm{Fe})^{5}\right)$ for the Sun by adopting the following effective temperature, surface gravity, and microturbulence velocity: $T_{\text {eff }}=5779 \mathrm{~K}, \log g=4.44$, and $\xi=0.8 \mathrm{~km} \mathrm{~s}^{-1}$. The values of the solar microturbulence reported in the literature range from 0.8 to $1.1 \mathrm{~km} \mathrm{~s}^{-1}$ (e.g. Randich et al. 2006). We chose $0.8 \mathrm{~km} \mathrm{~s}^{-1}$, which is the best fit value reported by Grevesse \& Sauval (1999); as well known, an increase in $\xi$ would result into a decrease of the solar $\log n(\mathrm{Fe})$. We derived $\log n(\mathrm{Fe} \mathrm{I})=7.49 \pm 0.04$ (standard deviation, or rms) and $\log n($ Fe II $)=7.54 \pm 0.03$, in good agreement with the estimates available in the literature (e.g., Grevesse \& Sauval 1999; Asplund et al. 2005). Usual checks for the suitability of the adopted parameters are the excitation equilibrium and the plot of Fe abundance vs. $E W$ s (see Sect. 4.4). We find a negligible trend of the Fe I abundance with $E P$ - hence the excitation equilibrium is satisfied (see Sect. 4.4) - while a slightly stronger trend is present considering $\log n(\mathrm{Fe} \mathrm{I})$ vs. the measured $E W \mathrm{~s}$ (a positive slope of $\sim 0.06$ in the plot of abundance vs. $\log (E W / \lambda)$ ). In order to put the value of this slope to $\sim 0$, we should have slightly enhanced the microturbulence (up to $0.95 \mathrm{~km} \mathrm{~s}^{-1}$ ) and, as a consequence, the $T_{\text {eff }}$ (up to $5800 \mathrm{~K}$ ); in this case, the Fe abundance would have been 7.48. However, we decided to retain the initial parameters for the Sun as the right ones and therefore did not put the slopes of $\log n(\mathrm{Fe} \mathrm{I})$ vs. measured $E W \mathrm{~s}$ and vs. $E P$ to zero. We warn the reader that this choice can lead to a systematic shift in the metallicity scale; had we assumed a lower $\log n(\mathrm{Fe} \mathrm{I})$, we would have found slightly higher metallicities for the open cluster stars.

Finally, we note that using the previous version (2002) of MOOG, where it is not possible to treat collisional damping with the Barklem coefficients, we would have obtained $\log n(\mathrm{Fe} \mathrm{I})=7.51 \pm 0.04$ and $\log n(\mathrm{Fe}$ II $)=7.55 \pm 0.04$ (considering classical damping constants from the Unsöld formula, in most cases multiplied by the enhancement factor $E$ ).

\subsection{Line list for giant stars}

The line list adopted for giant stars in open clusters (retrieved from G03) covers the wavelength range 5500-6800 $\AA$ for Fe I (157 lines) and 5500-6500 $\AA$ for Fe II (15 lines). The Fe features included were first carefully selected by G03 in order to exclude the presence of severe blending. Moreover, very strong lines $(E W \gtrsim 150 \mathrm{~m} \AA$, see Sect. 4.3$)$ have also been discarded, since they are critically sensitive to the microturbulence value; and further, a more detailed treatment of damping would be needed to fit the line wings. Note that, although the investigation by G03 concerned metal-poor halo stars, the line list adopted here was accurately tested and employed for solar metallicity open clusters (Carretta et al. 2004, 2005; B06a). Fe features at wavelengths bluer than $\sim 5500 \AA$ were excluded to avoid complications by crowding and continuum tracing: therefore, $\sim 60 \mathrm{Fe} \mathrm{I}$ and $\sim 25 \mathrm{Fe}$ II lines used for the solar analysis are not included in the giant list. On the other hand, there are additional useful

${ }^{5} \log n(\mathrm{Fe})=12+\log (\mathrm{N}(\mathrm{Fe}) / \mathrm{N}(\mathrm{H}))$, absolute number density abundance.
Fe I features with respect to the solar line list, in the list used for giants (since they are visible only in cool stars). Based on these facts, the analysis is not formally "differential" with respect to the Sun; however, as a test we measured the solar abundance using the lines shared by the two lists and found the same $\log n(\mathrm{Fe})$ values, reinforcing our method. More in detail, the fraction of Fe I features not included in the solar line list are about $25 \%$ of the lines used for giants. This, in principle, might represent a systematic bias, since the excitation equilibrium of $\mathrm{Fe} I$ lines (and therefore the $\left.T_{\text {eff }}\right)$ is strongly driven by the lowest and highest excitation lines. However, the majority of the features not shared by the Sun and giants have $E P$ in the range $3-5 \mathrm{eV}$, similar to those of the other lines present in the total lists (see also below). Only two lines in this subsample have very low EP $(<2 \mathrm{eV})$; therefore, the use of two different subsets of lines for the Sun and the giants does not represent a source of error.

Wavelengths, $E P$, and $\log g f$ for the lines used are reported in Table 8. A wide range in $E P$ and $\log g f$ (i.e., corresponding to a wide range of line strengths) is spanned at all wavelengths by Fe I features, with more than $70 \%$ of the lines having $E P \geq 3 \mathrm{eV}$ and $\log g f \geq-3$; only 10 features have $E P$ lower than $2 \mathrm{eV}$ and $\log g f$ lower than -4 .

The sources for the treatment of damping are also reported: G03 (see Sect. 4.1), Barklem et al. (2000), Unsöld (1955). Note that only in two cases (where the Barklem and G03 values were not available) was the classical Unsöld approximation used.

\subsection{Equivalent widths}

The continuum tracing and normalization of the spectra were carried out using the task CONTINUUM within IRAF, dividing the spectra in small regions (50 $\AA$ ) and visually checking the output. The $E W$ measurements were carried out with the program SPECTRE, developed by Chris Sneden (see Fitzpatrick \& Sneden 1987), which performs a Gaussian fitting of the line profiles. The values are available in electronic Tables 9-11, where the first two columns list the wavelengths and element - Fe I or Fe II - and the others show the corresponding $E W$ for each star. The $E W \mathrm{~s}$ which were not measurable (due to noise or blending) have been set to zero in the tables.

Continuum tracing and $E W$ determination are among the most critical steps in chemical abundance analysis, and they can represent the main reason for discrepancies between the results obtained by different authors (see Sect. 5). In particular, these steps are very problematic for metal-rich giant stars, which suffer from heavy line blending. We also mention that Gaussian fitting of the line profile is not always appropriate for strong lines; in those cases other fitting techniques, such as the use of a Voigt profile or a direct integration, would ensure that the contribution of damping wings is included in the measurement. Since the program SPECTRE does not allow performance of a fit different from Gaussian, we discarded lines with $E W$ s larger than $\sim 150 \mathrm{~m} \AA$ (with the exception of a couple of cases, see Fig. 5). On the other hand, the Gaussian function produces a good fit of the lines with $E W$ in the range 100-150 m $\AA$. This has been proved by measuring the lines with strength in this interval also with the task INTEGRATE/LINE within the program MIDAS, which performs a direct integration of the spectral features. We report in Fig. 5 a comparison between the $E W \mathrm{~s}$ measured with SPECTRE and MIDAS for three representative stars in the clusters. Note the very good agreement between the two sets of measurements for each star, indicating that the Gaussian fitting is appropriate for features not stronger than $\sim 150 \mathrm{~m} \AA$. 
Table 8. Set of Fe lines adopted for the analysis.

\begin{tabular}{|c|c|c|c|c|c|c|c|}
\hline $\begin{array}{l}\text { Wavelength } \\
(\AA)\end{array}$ & $\begin{array}{l}E P \\
(\mathrm{eV})\end{array}$ & $\log g f$ & $\begin{array}{l}\text { Damping } \\
\text { source }\end{array}$ & $\begin{array}{l}\text { Wavelength } \\
(\AA)\end{array}$ & $\begin{array}{l}E P \\
(\mathrm{eV})\end{array}$ & $\log g f$ & $\begin{array}{l}\text { Damping } \\
\text { source }\end{array}$ \\
\hline Fe I & & & & 5806.732 & 4.610 & -0.930 & 2 \\
\hline 5494.474 & 4.070 & -1.960 & 1 & 5811.912 & 4.140 & -2.360 & 1 \\
\hline 5521.281 & 4.430 & -2.510 & 1 & 5814.815 & 4.280 & -1.810 & 2 \\
\hline 5522.454 & 4.210 & -1.470 & 2 & 5835.109 & 4.260 & -2.180 & 2 \\
\hline 5524.244 & 4.150 & -2.840 & 2 & 5837.702 & 4.290 & -2.300 & 2 \\
\hline 5539.291 & 3.640 & -2.590 & 2 & 5849.687 & 3.690 & -2.950 & 2 \\
\hline 5547.000 & 4.220 & -1.850 & 2 & 5852.228 & 4.550 & -1.360 & 2 \\
\hline 5552.687 & 4.950 & -1.780 & 2 & 5853.150 & 1.480 & -5.090 & 1 \\
\hline 5560.220 & 4.430 & -1.100 & 2 & 5855.086 & 4.610 & -1.560 & 2 \\
\hline 5568.862 & 3.630 & -2.910 & 2 & 5856.096 & 4.290 & -1.570 & 2 \\
\hline 5577.028 & 5.030 & -1.490 & 1 & 5858.785 & 4.220 & -2.190 & 2 \\
\hline 5586.771 & 3.370 & -0.100 & 2 & 5859.596 & 4.550 & -0.630 & 1 \\
\hline 5587.581 & 4.140 & -1.700 & 1 & 5861.110 & 4.280 & -2.350 & 2 \\
\hline 5595.051 & 5.060 & -1.780 & 1 & 5862.368 & 4.550 & -0.420 & 1 \\
\hline 5608.976 & 4.210 & -2.310 & 2 & 5879.490 & 4.610 & -1.990 & 2 \\
\hline 5609.965 & 3.640 & -3.180 & 2 & 5880.025 & 4.560 & -1.940 & 2 \\
\hline 5611.357 & 3.630 & -2.930 & 2 & 5881.279 & 4.610 & -1.760 & 2 \\
\hline 5618.642 & 4.210 & -1.340 & 2 & 5902.476 & 4.590 & -1.860 & 2 \\
\hline 5619.609 & 4.390 & -1.490 & 2 & 5905.680 & 4.650 & -0.780 & 2 \\
\hline 5635.831 & 4.260 & -1.590 & 2 & 5927.797 & 4.650 & -1.070 & 2 \\
\hline 5636.705 & 3.640 & -2.530 & 2 & 5929.682 & 4.550 & -1.160 & 2 \\
\hline 5649.996 & 5.100 & -0.800 & 2 & 5930.191 & 4.650 & -0.340 & 2 \\
\hline 5651.477 & 4.470 & -1.790 & 2 & 5933.805 & 4.640 & -2.140 & 2 \\
\hline 5652.327 & 4.260 & -1.770 & 2 & 5934.665 & 3.930 & -1.080 & 2 \\
\hline 5661.017 & 4.580 & -2.420 & 2 & 5947.531 & 4.610 & -2.040 & 1 \\
\hline 5661.354 & 4.280 & -1.830 & 2 & 5956.706 & 0.860 & -4.560 & 2 \\
\hline 5677.689 & 4.100 & -2.640 & 2 & 5976.787 & 3.940 & -1.300 & 1 \\
\hline 5678.388 & 3.880 & -2.970 & 1 & 5984.826 & 4.730 & -0.290 & 1 \\
\hline 5680.244 & 4.190 & -2.290 & 1 & 6003.022 & 3.880 & -1.020 & 2 \\
\hline 5701.557 & 2.560 & -2.160 & 2 & 6007.968 & 4.650 & -0.760 & 1 \\
\hline 5717.841 & 4.280 & -0.980 & 2 & 6008.566 & 3.880 & -0.920 & 1 \\
\hline 5731.772 & 4.260 & -1.100 & 2 & 6015.242 & 2.220 & -4.660 & 2 \\
\hline 5738.240 & 4.220 & -2.240 & 2 & 6019.369 & 3.570 & -3.230 & 2 \\
\hline 5741.856 & 4.260 & -1.690 & 2 & 6027.059 & 4.070 & -1.200 & 1 \\
\hline 5742.963 & 4.180 & -2.350 & 2 & 6056.013 & 4.730 & -0.460 & 2 \\
\hline 5752.042 & 4.550 & -0.920 & 1 & 6065.494 & 2.610 & -1.490 & 2 \\
\hline 5754.406 & 3.640 & -2.850 & 2 & 6078.499 & 4.790 & -0.380 & 1 \\
\hline 5759.259 & 4.650 & -2.070 & 2 & 6079.016 & 4.650 & -0.970 & 2 \\
\hline 5760.359 & 3.640 & -2.460 & 2 & 6082.718 & 2.220 & -3.530 & 2 \\
\hline 5775.088 & 4.220 & -1.110 & 1 & 6089.574 & 5.020 & -0.870 & 1 \\
\hline 5778.463 & 2.590 & -3.440 & 2 & 6093.649 & 4.610 & -1.320 & 2 \\
\hline 5784.666 & 3.400 & -2.530 & 2 & 6094.377 & 4.650 & -1.560 & 2 \\
\hline 5793.922 & 4.220 & -1.620 & 2 & 6096.671 & 3.980 & -1.760 & 2 \\
\hline
\end{tabular}

\subsection{Stellar parameters}

Initial effective temperatures were derived from $B, V$, and $K$ photometry, applying the calibration by Alonso et al. (1999), based on a large sample of field and open-cluster giant stars. The initial surface gravity was computed as $\log g=\log \left(M / M_{\odot}\right)+$ $0.4\left(M_{\text {bol }}-M_{\text {bol } \odot}\right)+4 \cdot \log \left(T_{\text {eff }} / T_{\text {eff } \odot}\right)+\log g_{\odot}$, where $M$ is the mass and $M_{\text {bol }}$ the bolometric magnitude (with the symbol $\odot$ referring to the Sun and $M_{\text {bol } \odot}=4.72$ ). The clump masses were retrieved from the isochrones computed by the Padova group (Bertelli et al. 1994): $1 M_{\odot}$ for Be 32 (age $\sim 6 \mathrm{Gyr}$ ), and $2 M_{\odot}$ for NGC 3960 and NGC 2660 (age 1 Gyr). We adopted indicative ages, but the surface gravity is only slightly affected by the choice of clump mass: for example, assuming $1.1 M_{\odot}$ instead of $1.0 M_{\odot}$ would imply a change of $\sim 0.04$ dex in $\log g$.

In addition, the photometric $T_{\text {eff }}$ and $\log g$ only represent starting values, whereas the two parameters were optimized during the spectral analysis. More specifically, we employed the driver ABFIND in MOOG to compute Fe abundances for the stars: the final effective temperature was chosen in order to eliminate possible trends in $\log n(\mathrm{Fe} \mathrm{I})$ vs. $E P$ (excitation equilibrium). As is well known, this method relies on the circumstance that, when the $T_{\mathrm{eff}}$ is over-estimated, the observed $E W \mathrm{~s}$ of the lines with higher EP are matched by a lower abundance, and vice versa.

The surface gravity was optimized by assuming the ionization equilibrium condition, i.e. $\log n(\mathrm{Fe}$ II $)-\log n(\mathrm{Fe} \mathrm{I})=0.05$ (as found for the Sun). Then, if necessary the $T_{\text {eff }}$ was re-adjusted in order to satisfy both the ionization and excitation equilibria.

The choice of the microturbulence velocity deserves a more detailed description. In most of the chemical analysis present in the literature $\xi$ is optimized by minimizing the slope of the relationship between $\log n(\mathrm{Fe} \mathrm{I})$ and the observed $E W \mathrm{~s}$. This technique is based on the fact that strong lines are very sensitive to microturbulence: therefore, too high a $\xi$ would yield too low an abundance for strong lines. On the other hand, 
Table 8. continued.

\begin{tabular}{|c|c|c|c|c|c|c|c|}
\hline $\begin{array}{l}\text { Wavelength } \\
(\AA)\end{array}$ & $\begin{array}{l}E P \\
(\mathrm{eV})\end{array}$ & $\overline{\log g f}$ & $\begin{array}{l}\text { Damping } \\
\text { source }\end{array}$ & $\begin{array}{l}\text { Wavelength } \\
(\AA)\end{array}$ & $\begin{array}{l}E P \\
(\mathrm{eV})\end{array}$ & $\log g f$ & $\begin{array}{l}\text { Damping } \\
\text { source }\end{array}$ \\
\hline 6098.250 & 4.560 & -1.810 & 2 & 6581.218 & 1.480 & -4.680 & 2 \\
\hline 6120.258 & 0.910 & -5.860 & 1 & 6591.314 & 4.590 & -2.040 & 2 \\
\hline 6137.002 & 2.200 & -2.910 & 2 & 6593.884 & 2.430 & -2.300 & 2 \\
\hline 6151.623 & 2.180 & -3.260 & 2 & 6608.044 & 2.280 & -3.960 & 2 \\
\hline 6157.733 & 4.070 & -1.260 & 1 & 6609.118 & 2.560 & -2.650 & 2 \\
\hline 6165.363 & 4.140 & -1.480 & 1 & 6625.039 & 1.010 & -5.320 & 1 \\
\hline 6173.341 & 2.220 & -2.840 & 2 & 6627.560 & 4.550 & -1.500 & 2 \\
\hline 6187.402 & 2.830 & -4.130 & 1 & 6633.758 & 4.560 & -0.810 & 2 \\
\hline 6187.995 & 3.940 & -1.600 & 2 & 6667.426 & 2.450 & -4.370 & 2 \\
\hline 6199.509 & 2.560 & -4.350 & 2 & 6667.723 & 4.580 & -2.100 & 2 \\
\hline 6200.321 & 2.610 & -2.390 & 2 & 6699.142 & 4.590 & -2.110 & 2 \\
\hline 6213.437 & 2.220 & -2.540 & 2 & 6703.576 & 2.760 & -3.000 & 2 \\
\hline 6219.287 & 2.200 & -2.390 & 2 & 6704.485 & 4.220 & -2.640 & 1 \\
\hline 6220.791 & 3.880 & -2.360 & 2 & 6713.745 & 4.790 & -1.410 & 2 \\
\hline 6226.740 & 3.880 & -2.080 & 2 & 6725.364 & 4.100 & -2.210 & 2 \\
\hline 6232.648 & 3.650 & -1.210 & 3 & 6726.673 & 4.610 & -1.050 & 1 \\
\hline 6240.653 & 2.220 & -3.230 & 2 & 6733.153 & 4.640 & -1.440 & 2 \\
\hline 6246.327 & 3.600 & -0.730 & 2 & 6739.524 & 1.560 & -4.850 & 2 \\
\hline 6252.565 & 2.400 & -1.640 & 2 & 6745.965 & 4.070 & -2.710 & 1 \\
\hline 6265.141 & 2.180 & -2.510 & 2 & 6750.164 & 2.420 & -2.580 & 2 \\
\hline 6270.231 & 2.860 & -2.550 & 2 & 6753.465 & 4.560 & -2.350 & 2 \\
\hline 6280.622 & 0.860 & -4.340 & 2 & 6756.547 & 4.290 & -2.780 & 1 \\
\hline 6290.548 & 2.590 & -4.360 & 2 & 6786.860 & 4.190 & -1.900 & 2 \\
\hline 6297.799 & 2.220 & -2.700 & 2 & 6793.260 & 4.070 & -2.430 & 1 \\
\hline 6301.508 & 3.650 & -0.720 & 3 & 6796.120 & 4.140 & -2.400 & 1 \\
\hline 6303.466 & 4.320 & -2.620 & 2 & 6804.297 & 4.580 & -1.850 & 2 \\
\hline 6311.504 & 2.830 & -3.160 & 2 & 6806.856 & 2.730 & -3.140 & 2 \\
\hline 6315.814 & 4.070 & -1.670 & 1 & 6810.267 & 4.610 & -1.000 & 2 \\
\hline 6322.694 & 2.590 & -2.380 & 2 & Fe II & & & \\
\hline 6330.852 & 4.730 & -1.220 & 2 & 5525.135 & 3.270 & -4.040 & 2 \\
\hline 6335.337 & 2.200 & -2.280 & 2 & 5534.848 & 3.240 & -2.750 & 2 \\
\hline 6380.750 & 4.190 & -1.340 & 1 & 5627.502 & 3.390 & -4.140 & 2 \\
\hline 6392.538 & 2.280 & -3.970 & 2 & 5991.378 & 3.150 & -3.550 & 2 \\
\hline 6393.612 & 2.430 & -1.430 & 2 & 6084.105 & 3.200 & -3.800 & 2 \\
\hline 6400.323 & 3.600 & -0.230 & 1 & 6113.329 & 3.210 & -4.120 & 2 \\
\hline 6411.108 & 4.730 & -2.330 & 1 & 6149.249 & 3.890 & -2.720 & 2 \\
\hline 6411.658 & 3.650 & -0.600 & 2 & 6239.948 & 3.890 & -3.440 & 2 \\
\hline 6421.360 & 2.280 & -1.980 & 2 & 6247.562 & 3.870 & -2.320 & 2 \\
\hline 6436.411 & 4.190 & -2.400 & 1 & 6369.463 & 2.890 & -4.210 & 2 \\
\hline 6481.878 & 2.280 & -2.940 & 2 & 6383.715 & 5.550 & -2.090 & 2 \\
\hline 6498.945 & 0.960 & -4.660 & 2 & 6416.928 & 3.890 & -2.700 & 2 \\
\hline 6518.373 & 2.830 & -2.560 & 2 & 6432.683 & 2.890 & -3.580 & 2 \\
\hline 6533.940 & 4.560 & -1.280 & 2 & 6456.391 & 3.900 & -2.100 & 2 \\
\hline 6574.254 & 0.990 & -4.960 & 1 & 6516.083 & 2.890 & -3.380 & 2 \\
\hline
\end{tabular}

References: (1) Gratton et al. (2003; G03); (2) Barklem et al. (2000); (3) Unsöld (1955).

Magain (1984) showed that random errors in $E W$ s lead to systematic over-estimation of the microturbulence. A possible method of obtaining an unbiased determination of $\xi$ is to optimize it by instead using the expected $E W \mathrm{~s}$, which are free from random errors. The theoretical $E W \mathrm{~s}$ can be computed as $\log E W_{\text {exp }}=\log g f-E P \cdot\left(5040 /\left(0.86 \cdot T_{\text {eff }}\right)\right) \mathrm{m} \AA$, which is an approximation of a classical expression (Gray 1976).

For simplicity and for self-consistency, we adopted a relation derived using the following procedure by Carretta et al. (2004), who analyzed a sample of stars in three open clusters with nearly solar metallicity and derived a relationship between the final microturbulence velocities (optimized using the method suggested by Magain 1984) and $\log g$. The resulting expression is $\xi=[1.5-0.13 \times \log g] \mathrm{km} \mathrm{s}^{-1}$. We tested this relationship for our sample of stars: namely, we constructed plots of $\log n(\mathrm{Fe} \mathrm{I})$ vs. expected $E W \mathrm{~s}$ and found that the microturbulence obtained by zeroing the slope of this relationship is very similar to that which is computed following the prescriptions by Carretta et al. In fact, considering the whole sample of stars in the three clusters, we obtained a relationship between $\xi$ and $\log g$ that is almost identical to the expression reported above.

We also performed a comparison with a more commonly used analysis by optimizing the microturbulences using the $\log n(\mathrm{Fe} \mathrm{I})$ vs. observed $E W \mathrm{~s}$. We find reasonable differences between the final atmospheric parameters derived with the two techniques: in the worst cases we have $\Delta \xi \sim 0.15 \mathrm{~km} \mathrm{~s}^{-1}$, $\Delta T_{\text {eff }} \sim 80 \mathrm{~K}, \Delta \log g \sim 0.3 \mathrm{dex}$, but generally average differences in $T_{\text {eff }}$, microturbulence, and gravity do not exceed $50 \mathrm{~K}, 0.1 \mathrm{~km} \mathrm{~s}^{-1}$, and $0.2 \mathrm{dex}$, respectively. Variations in the atmospheric parameters concur in different ways to changes in abundances, and the differences in the final Fe content are within \pm 0.02 dex. Furthermore, we notice that in some cases the 
Table 12. Stellar parameters (photometric and spectroscopic) and Fe abundances for the sample stars in the three clusters.

\begin{tabular}{|c|c|c|c|c|c|c|c|c|c|}
\hline Star & $\begin{array}{l}T_{\text {eff }(B-V)} \\
(\mathrm{K})\end{array}$ & $\begin{array}{l}T_{\text {eff }(V-K)} \\
(\mathrm{K})\end{array}$ & $\log g_{\text {phot }} *$ & $\begin{array}{l}T_{\text {eff spec }} \\
(\mathrm{K})\end{array}$ & $\log g_{\text {spec }}$ & $\begin{array}{l}\xi \\
\mathrm{km} \mathrm{s}^{-1}\end{array}$ & {$[\mathrm{Fe} / \mathrm{H}]$} & $\sigma_{1}$ & $\sigma_{\text {tot }}$ \\
\hline \multicolumn{10}{|l|}{ NGC 3960} \\
\hline c3 & 4814 & 4743 & 2.78 & 4950 & 2.35 & 1.19 & +0.00 & 0.08 & 0.09 \\
\hline c4 & 5047 & 5027 & 2.78 & 5050 & 2.54 & 1.17 & +0.07 & 0.07 & 0.09 \\
\hline c5 & 4862 & 4769 & 2.62 & 4870 & 2.16 & 1.22 & +0.00 & 0.07 & 0.09 \\
\hline c6 & 5120 & 5121 & 2.72 & 4950 & 2.4 & 1.19 & +0.02 & 0.08 & 0.09 \\
\hline c8 & 5064 & 5017 & 2.73 & 5040 & 2.57 & 1.18 & +0.00 & 0.07 & 0.09 \\
\hline c9 & 4968 & 4959 & 2.71 & 5000 & 2.45 & 1.18 & +0.02 & 0.08 & 0.09 \\
\hline Average $\mathrm{Fe}$ & & & & & & & +0.02 & & 0.04 (rms) \\
\hline \multicolumn{10}{|l|}{$\mathrm{Be} 32$} \\
\hline 17 & 4830 & 4738 & 2.45 & 4830 & 2.22 & 1.21 & -0.31 & 0.07 & 0.11 \\
\hline 18 & 4866 & 4784 & 2.47 & 4850 & 2.27 & 1.21 & -0.27 & 0.08 & 0.12 \\
\hline 19 & 4822 & 4694 & 2.45 & 4760 & 2.26 & 1.21 & -0.35 & 0.08 & 0.12 \\
\hline 25 & 4718 & 4598 & 2.61 & 4760 & 2.40 & 1.19 & -0.20 & 0.10 & 0.13 \\
\hline 27 & 4729 & 4622 & 2.67 & 4780 & 2.35 & 1.19 & -0.24 & 0.08 & 0.12 \\
\hline 45 & 4964 & 4905 & 3.15 & 4920 & 3.00 & 1.11 & -0.35 & 0.10 & 0.13 \\
\hline 938 & - & 4732 & 2.45 & 4870 & 2.33 & 1.19 & -0.30 & 0.09 & 0.13 \\
\hline 940 & 4800 & 4710 & 2.45 & 4800 & 2.10 & 1.23 & -0.33 & 0.09 & 0.13 \\
\hline 941 & 4793 & 4690 & 2.43 & 4760 & 2.40 & 1.19 & -0.29 & 0.08 & 0.13 \\
\hline Average $\mathrm{Fe}$ & & & & & & & -0.29 & & $0.04(\mathrm{rms})$ \\
\hline \multicolumn{10}{|l|}{ NGC 2660} \\
\hline 296 & 5126 & - & 3.01 & 5200 & 3.01 & 1.11 & +0.08 & 0.09 & 0.12 \\
\hline 318 & 5028 & 4926 & 2.75 & 5030 & 2.59 & 1.16 & 0.00 & 0.07 & 0.11 \\
\hline 542 & 5008 & 4925 & 2.76 & 5060 & 2.48 & 1.17 & +0.03 & 0.07 & 0.11 \\
\hline 694 & 4996 & 4886 & 2.85 & 5100 & 2.77 & 1.14 & +0.05 & 0.08 & 0.11 \\
\hline 862 & 5036 & 4940 & 2.85 & 5100 & 2.60 & 1.16 & +0.02 & 0.09 & 0.12 \\
\hline Average $\mathrm{Fe}$ & & & & & & & +0.04 & & 0.04 (rms) \\
\hline
\end{tabular}

* Photometric gravities are the average between $\log g$ from $(B-V)$ and $(V-K)$.

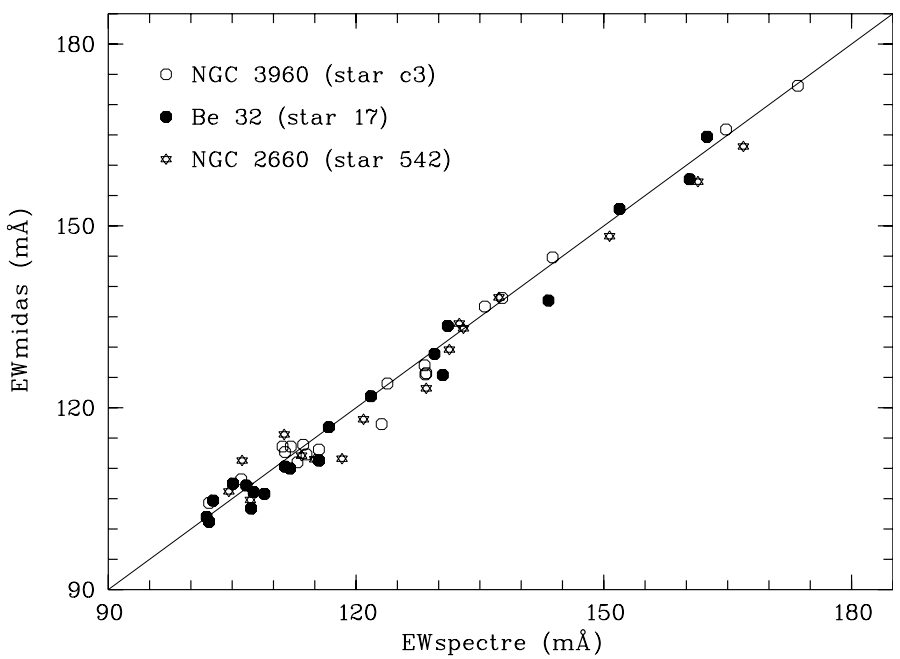

Fig. 5. Comparison between $E W$ measured with SPECTRE and with MIDAS for strong Fe I lines in sample stars of the three clusters.

relationship $\log n(\mathrm{Fe} \mathrm{I})$ vs. observed $E W$ has a negligible slope, even if the microturbulence has been optimized in function of the expected $E W \mathrm{~s}$.

\subsection{Errors}

Our abundance scale is directly referred to the solar $\log n(\mathrm{Fe})$ and the majority of the spectral lines used for giants stars are in common with those included in the solar line list. In this way, internal errors due to uncertainties in the oscillator strengths should be minimized.
Random internal errors in $E W$ s can be estimated by comparing the $E W$ s of two stars with similar parameters. Considering the common lines between the two stars and rejecting significant outliers, we found the following average differences: $\langle\Delta E W\rangle \pm \delta=-0.95 \pm 2.54 \mathrm{~m} \AA$ (where $\delta$ indicates the rms) for NGC 3960 (c3-c6; 112 lines), -1.02 $\pm 3.75 \mathrm{~m} \AA$ for Be 32 (17-794; 90 lines), and $-1.49 \pm 4.48 \mathrm{m \AA}$ for NGC 2660 (318-862; 76 lines). The random errors in $E W$ are represented by $\delta / \sqrt{2}$ (assuming that they can be equally attributed to both stars in the pair under consideration), therefore we find that their values are $\sim 1.8, \sim 2.7$, and $\sim 3.2 \mathrm{~m} \AA$ for NGC 3960, Be 32 and NGC 2660, respectively.

The effect of random errors in EWs and of errors in the atomic parameters on the derived abundance for a single star is well-represented by $\sigma_{1}$, the standard deviation from the mean abundance based on the whole set of lines (see Table 12). However, $\mathrm{Fe}$ abundances are also affected by uncertainties on the adopted stellar parameters: the total error in $[\mathrm{Fe} / \mathrm{H}]$ for each star, $\sigma_{\text {tot }}$, can be computed by quadratically adding $\sigma_{1}$ to the error deriving from random uncertainties in $T_{\text {eff }}, \log g$ and $\xi$, which we will call $\sigma_{2}$. Typical $\sigma_{2}$ values for each cluster can be estimated by varying one parameter at a time (holding the others fixed) and then by quadratically adding the three related errors, $\sigma_{T \text { eff-rd }}, \sigma_{\log g \text {-rd }}$, and $\sigma_{\xi \text {-rd }}$ (see below).

Errors in $T_{\mathrm{eff}}, \log g$, and $\xi$ have been evaluated in the same fashion as in Carretta et al. (2004). A detailed description of the method can be found in the quoted reference, but we mention here that we find standard total internal errors of $\sim 35 \mathrm{~K}$ in temperature for individual stars in the three clusters. The corresponding uncertainty in abundance $\left(\sigma_{\text {Teff-TOT }}\right)$ is the quadratic sum of the internal random term $\left(\sigma_{T \text { eff-rd }}\right.$, related to the $E W$ measurement) and the internal systematic term $\left(\sigma_{T \text { eff-sys }}\right)$. Using the formulas of Carretta et al., we estimated $\sigma_{T \text { eff-rd }}$ to be 0.033 , 
Table 13. Sensitivities of $\mathrm{Fe}$ abundances to variations in the atmospheric parameters for typical stars in the three clusters.

\begin{tabular}{llll}
\hline \hline Star & $\sigma_{T \text { eff }}$ & $\sigma_{\log g}$ & $\sigma_{\xi}$ \\
& $\Delta T_{\text {eff }}= \pm 100 \mathrm{~K}$ & $\Delta \log g= \pm 0.2 \mathrm{dex}$ & $\Delta \xi= \pm 0.15 \mathrm{~km} \mathrm{~s}^{-1}$ \\
\hline c9 (NGC 3960) & $+0.09 /-0.06$ & $+0.01 / 0.00$ & $-0.05 /+0.06$ \\
17 (Be 32) & $+0.09 /-0.07$ & $+0.01 / 0.00$ & $-0.05 /+0.06$ \\
542 (NGC 2660) & $+0.09 /-0.07$ & $+0.01 / 0.00$ & $-0.05 /+0.06$ \\
\hline
\end{tabular}

0.055, and 0.066 dex for NGC 3960, NGC 2660, and Be 32, respectively, i.e. about $47 \%, 69 \%$, and $78 \%$ of the total uncertainty. Therefore, the internal random errors in the temperatures are $\sim 15-25 \mathrm{~K}$ for individual stars in these clusters. Systematic scale errors are always difficult to estimate, and a more detailed discussion must be deferred to the completion of the analysis for our whole sample of clusters. At present, the good agreement between results from different approaches (photometric and spectroscopic) allows us to estimate that scale errors are likely to be confined typically within $100-150 \mathrm{~K}$.

Errors in surface gravities are due to two contributions, one from spectroscopic effective temperatures, and the other from errors in the measurement of individual lines (see Carretta et al. 2004). The total (random + systematic) error $\Delta \log g$ results in $0.15-0.25$ dex. Taking only the random part (i.e. 47, 69, and $78 \%$ for NGC 3960, NGC 2660, and Be 32, respectively) of this error into account, we find that the total random uncertainty $\Delta \log g_{\mathrm{rd}}$ is 0.13 dex for NGC 3960, 0.10 dex for NGC 2660 and 0.12 dex for Be 32 .

Errors in the microturbulence velocity are computed for a typical star in each cluster and depend on the variation in the slope of the adopted abundance vs. expected $E W$ relation and on the relationship between $\xi$ and $\log g$ (see Carretta et al. 2004). The random internal errors $\Delta \xi_{\text {rd }}$ are $\sim 0.10 \mathrm{~km} \mathrm{~s}^{-1}$ for NGC 3960 , $\sim 0.17 \mathrm{~km} \mathrm{~s}^{-1}$ for NGC 2660 and $\sim 0.20 \mathrm{~km} \mathrm{~s}^{-1}$ for Be 32 .

As already mentioned, $\sigma_{2}$ is the error in abundance related to random internal uncertainties in the stellar parameters, and it is due to three terms: $\sigma_{T \text { eff-rd }}, \sigma_{\log g \text {-rd }}$, and $\sigma_{\xi \text {-rd }}$. The first term is reported above, while the two last are calculated by estimating the sensitivity of $\log n(\mathrm{Fe} \mathrm{I})$ to the following changes: $\Delta \log g_{\mathrm{rd}}=0.10-0.13 \mathrm{dex}$ and $\Delta \xi_{\mathrm{rd}}=0.10-0.20 \mathrm{~km} \mathrm{~s}^{-1}$. The sensitivity of $[\mathrm{Fe} / \mathrm{H}]$ to errors in the stellar parameters is exemplified in Table 13: for each cluster we chose the star with Fe abundance and $T_{\text {eff }}$ most similar to the average values. Note that we adopted sample parameter variations of $\pm 100 \mathrm{~K}$ in $T_{\text {eff }}, \pm 0.2$ dex in $\log g$ and $\pm 0.15 \mathrm{~km} \mathrm{~s}^{-1}$ in $\xi$, even if different from the random errors in the stellar parameters estimated to compute $\sigma_{2}$ in our clusters.

The presence of systematic errors due to the method of analysis can be checked by analyzing stars with well-known metallicity, as for example the Hyades, and possibly observed with the same instrument. Unfortunately, we observed two Hyades stars (the clump objects $\gamma$ Tau and $\delta$ Tau) only with SARG at the TNG at lower resolution $(R \sim 29000)$. The same method described above was employed to carry out the analysis. We found similar abundances and parameters for the two stars, with $T_{\text {eff }}=4860 \mathrm{~K}$, $\xi=1.40 \mathrm{~km} \mathrm{~s}^{-1}, \log g=2.50$, and $[\mathrm{Fe} / \mathrm{H}]=+0.17 \pm 0.08$ for $\gamma \mathrm{Tau}$, and $T_{\text {eff }}=4905 \mathrm{~K}, \xi=1.41 \mathrm{~km} \mathrm{~s}^{-1}, \log g=2.60$, and $[\mathrm{Fe} / \mathrm{H}]=+0.19 \pm 0.08$ for $\delta$ Tau. Then $\xi$ was optimized using both the observed and expected $E W$. We note that using the parameters reported above we were indeed able to optimize the $\mathrm{Fe}$ abundance vs. $E W \mathrm{~s}$ distribution for either expected and observed $E W$ s.

Our value for the metallicity of the Hyades appears somewhat higher than most of the estimates present in the literature based on dwarfs: for example Boesgaard \& Friel (1990) quoted $[\mathrm{Fe} / \mathrm{H}]=+0.13$ for MS stars, which is the most commonly accepted value. However, considering that they assume $\log n(\mathrm{Fe})_{\odot}=7.56$, higher by 0.07 than our value, our metallicity is perfectly consistent with theirs. In more recent analysis Boesgaard et al. (2002) quote $[\mathrm{Fe} / \mathrm{H}]=+0.16$, while Paulson et al. (2003) find $[\mathrm{Fe} / \mathrm{H}]=+0.13$. Recent investigations of giant stars are those by Smith $(1999:[\mathrm{Fe} / \mathrm{H}]=+0.15)$, by Wylie et al. $(2004:[\mathrm{Fe} / \mathrm{H}]=+0.19)$, and by Schuler et al. (2006: $[\mathrm{Fe} / \mathrm{H}]=+0.16)$, in agreement with our result.

\section{Results}

Our results for the Fe abundances are reported in Table 12. Columns 2 and 3 list the photometric $T_{\text {eff }}$ derived from $(B-V)$ and $(V-K)$ colors, and the average of these two values was assumed as initial $T_{\text {eff }}$ for the analysis for each star. In Col. 4 we report the photometric $\log g$ (mean value of gravities from $B-V$ and $V-K$ colors). For star 938 in Be $32 B V$ photometry by D'Orazi et al. (2006) was not available; therefore, we adopted the $V$ magnitude by Kaluzny \& Mazur (1991) and listed only $T_{\text {eff }}(V-K)$. For star 296 in NGC 2660, the $K$ value is clearly wrong, leading to a $T_{\text {eff }}(V-K)$ of $4300 \mathrm{~K}$, very different from the $T_{\mathrm{eff}}(B-V)$ and from the temperatures of the other clump stars. For the whole sample, effective temperatures derived from $(B-V)$ and $(V-K)$ colors are in generally good agreement (i.e. within the errors), differing by $0-70 \mathrm{~K}$ in NGC 3960, by 20-150 K in Be 32, and by $100 \mathrm{~K}$ in NGC 2660.

The final stellar parameters determined via the spectroscopic analysis described in Sect. 4.4 are shown in Cols. 5-7 $\left(T_{\text {eff }}\right.$, $\log g$ and $\xi$ ). In most cases, we found reasonable agreement between the photometric and spectroscopic parameters. The spectroscopic and (average) photometric temperatures usually coincide within $\sim 100 \mathrm{~K}$.

As far as surface gravities are concerned, we note that to satisfy the ionization equilibrium, we had to consider a $\log g$ usually lower than the photometric ones. The differences have average values of $\Delta\left(\log g_{\text {phot }}-\log g_{\text {spec }}\right)=0.30 \pm 0.12$ (statistical error) for NGC $3960,0.20 \pm 0.10$ for Be 32 , and $0.15 \pm 0.12$ for NGC 2660. These differences are slightly larger than the errors in $\log g$ (see Sect. 4.5) and could be attributed to several random factors, such as internal errors, errors in distance moduli ( 0.2 mag translates into 0.08 dex in gravity), errors in reddenings (important especially for NGC 3960 where a differential reddening was noted by Prisinzano et al. 2004 and confirmed for the inner region by B06a at the level of \pm 0.05 mag, corresponding to $\Delta \log g \sim 0.07 \mathrm{dex}$ ), errors in ages (i.e., in masses, although the contribution is rather small), non homogeneity of the data sources, etc. Another possible explanation for the difference between spectroscopic and photometric gravities might be represented by non-LTE effects and/or inadequacies in classical (1-d) model atmospheres where important features such as spots, granulation, activity, etc. are neglected. Such a discrepancy has also been encountered by other authors (Feltzing \& Gustafsson 1998; Schuler et al. 2003; Allende Prieto et al. 2004) in studies of cool metal-rich stars. Nevertheless, random uncertainties 

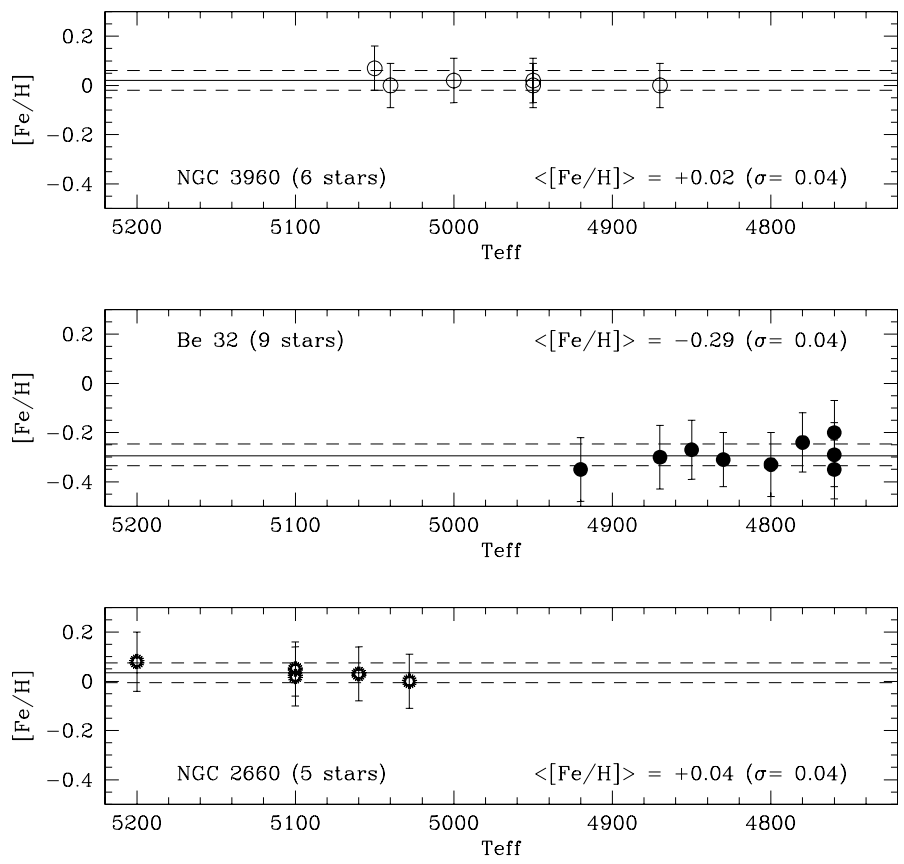

Fig. 6. Fe abundances $([\mathrm{Fe} / \mathrm{H}])$ as a function of effective temperature for stars in the three clusters. From top to bottom: NGC 3960, Be 32 and NGC 2660. Error bars $\left(\sigma_{\text {tot }}\right.$, see Sect. 5) are also shown. The solid lines represent the average $[\mathrm{Fe} / \mathrm{H}]$ (weighted mean) for the cluster, while the dashed ones indicate the standard deviation from the mean (rms).

might not be the only reason for the discrepancies between photometric and spectroscopic gravities, as also a systematic error due to the method of analysis could probably be present. The microturbulence values, derived as described in Sect. 4.4, cover a rather narrow range in each cluster, as expected from the fact that clump stars are in the same evolutionary status.

In the last three columns of Table 12, we report the Fe abundance with respect to the $\operatorname{Sun}([\mathrm{Fe} / \mathrm{H}])$, and their errors: $\sigma_{1}$ and $\sigma_{\text {tot }} . \sigma_{\text {tot }}$ are computed by quadratically adding $\sigma_{1}$ and $\sigma_{2}$ as discussed in Sect. 4.5. We estimated typical $\sigma_{2}$ values in the three clusters of $\sim 0.05$ dex for NGC 3960, and 0.08-0.09 dex for the other two clusters. When determining Fe abundances of the stars, $1 \sigma$-clipping was performed as the first step, that is, before the optimization of the stellar parameters.

The average (weighted mean) values for the clusters are also given, with the standard deviation from the mean (considering the total uncertainty $\sigma_{\text {tot }}$ ). NGC 3960 turns out to have a solar metallicity, $\langle[\mathrm{Fe} / \mathrm{H}]\rangle=+0.02 \pm 0.04 \mathrm{dex}$, at variance with some previous reports of sub-solar Fe content (see the discussion in Sect. 6.1). NGC 2660 also has a solar Fe content: $\langle[\mathrm{Fe} / \mathrm{H}]\rangle=$ $+0.04 \pm 0.04$, while for Be 32 we derive a mean value $\langle[\mathrm{Fe} / \mathrm{H}]\rangle=$ $-0.29 \pm 0.04$ in fair agreement with previous estimates of subsolar metallicity.

Plots of the Fe abundance as a function of $T_{\text {eff }}$ are shown in Fig. 6 for each cluster. Error bars $\left(\sigma_{\text {tot }}\right)$ are also reported. The resulting abundances are characterized by a small dispersion. This is only in part due to the 1- $\sigma$ clipping performed during the first iteration of the analysis; indeed, most of the lines discarded during that step were common to the various stars. Typical $\sigma_{1}$ values before the clipping were about 0.15 dex (i.e. twice the final $\sigma_{1}$ ).

\section{Discussion}

\subsection{Comparison with previous studies}

Using the synthetic CMD technique, Sandrelli et al. (1999) found for NGC 2660 that all the best-fit models for the three adopted sets of stellar evolutionary tracks were obtained for solar metallicity. For Be 32, Friel et al. (2002) derived $[\mathrm{Fe} / \mathrm{H}]=-0.50$ from low-resolution spectra. Sub-solar metallicity has also been found by Kaluzny \& Mazur (1991) on the basis of photometry $([\mathrm{Fe} / \mathrm{H}]=-0.37 \pm 0.05)$ and by D'Orazi et al. (2006) who derived a best-fit metallicity $Z=0.008([\mathrm{Fe} / \mathrm{H}] \sim-0.40)$. While our results are reasonably consistent with the previous ones, it is important to emphasize that we provide the first metallicity reports for these two open clusters based on high-resolution spectroscopic data.

As far as NGC 3960 is concerned, literature reports based on photometry or low resolution spectroscopy quoted a clearly sub-solar Fe content (Friel \& Janes 1993; Geisler et al. 1992; Piatti et al. 1995). The discrepancy with these results is probably due to the difference in the reliability of the method of analysis and quality of the data, so we think it is not worth undertaking a detailed comparison. On the other hand, we can perform a direct comparison with the very recent study by B06a, who carried out a spectroscopic analysis (Fe and other elements) for three stars in the cluster, observed with the FEROS spectrograph at the $1.5 \mathrm{~m}$ ESO telescope in La Silla (Chile). The three objects in common with our sample are c5, c6 and c8. While the $S / N$ ratio of their spectra is lower than ours, the spectral resolving power of the two instruments is comparable.

The same line list (with the same $\log g f$ and damping parameters) was used to determine the Fe abundances; furthermore, the same concepts for optimizing the stellar parameters were adopted. Nevertheless, the two methods of analysis differ in other important steps: the determination of the continuum and $E W$ measurement and the adopted spectral code (the package ROSA, developed by Gratton 1988; has been used by B06a). Detailed descriptions of the method of analysis adopted by B06a can be found in Bragaglia et al. (2001), G03, and Carretta et al. (2004).

The comparison between our results and those by B06a is presented in Table 14, where a very good agreement between the spectroscopic parameters derived by the two methods is evident. In the last column we list the difference among the net $\log n(\mathrm{Fe})$ values from the two studies. Note that B06a have $\log n(\mathrm{Fe} \mathrm{I})_{\odot}=7.54$ and $\log n(\mathrm{Fe} \text { II })_{\odot}=7.49$ for the Sun (while we find Fe II to be higher than Fe I, see Sect. 4.1). We carefully checked the reasons for this difference between the solar Fe content derived with MOOG and ROSA: in the case of neutral Fe, the abundance obtained with ROSA for the Sun is systematically higher, for both strong and weak lines. Had we found higher differences in the case of strong lines, this could have been related to a faulty treatment of damping. The difference between the abundances of ionized Fe goes instead in the opposite direction with respect to Fe I. We therefore tentatively conclude that the discrepancy of \pm 0.05 dex among the two solar analyses could be due to a systematic difference between the two combinations of spectroscopic code and model atmospheres. Considering this, the average difference between the metallicity obtained by us and by B06a for the three stars in common is 0.07 dex (our estimate minus the B06a one).

By comparing the $E W$ sets for each star, we found that our measurements are systematically higher than those of B06a, with average differences of $\sim 2-5 \mathrm{~m} \AA(\sigma \sim \pm 6 \mathrm{~m} \AA)$. The reason for 
Table 14. NGC 3960: comparison with the results by Bragaglia et al. (2006a); numbers in parenthesis are their IDs.

\begin{tabular}{lllllll}
\hline \hline star & & $\begin{array}{l}T_{\text {eff }} \\
\mathrm{K}\end{array}$ & $\log g$ & $\begin{array}{l}\xi \\
\mathrm{km} \mathrm{s}^{-1}\end{array}$ & {$[\mathrm{Fe} / \mathrm{H}]$} & $\Delta \log n(\mathrm{Fe})$ \\
\hline c5 & MOOG & 4870 & 2.16 & 1.22 & +0.00 & -0.09 \\
(41) & ROSA & 4850 & 2.20 & 1.21 & -0.14 & \\
& & & & & & \\
c6 & MOOG & 4950 & 2.40 & 1.19 & +0.02 & -0.12 \\
$(28)$ & ROSA & 4900 & 2.06 & 1.23 & -0.15 & \\
& & & & & & \\
c8 & MOOG & 5040 & 2.57 & 1.17 & +0.00 & -0.01 \\
$(50)$ & ROSA & 5000 & 2.70 & 1.15 & -0.06 & \\
\hline
\end{tabular}

such a discrepancy should be attributed to the determination of the continuum and the method of measurement. In order to check this hypothesis, we performed a complete re-analysis (i.e. starting from the normalization of the spectrum) of the FEROS spectrum of star c6 used by B06a, for which the largest difference $\Delta[\mathrm{Fe} / \mathrm{H}]_{\text {MOOG-ROSA }}$ is observed. We considered only the portion of the spectrum in common with the UVES range and found the FEROS and UVES $E W$ sets to be nearly identical (average difference $\sim 1.5 \mathrm{~m} \AA$, instead of $\sim 3 \mathrm{~m} \AA$ when we use the original B06a $E W \mathrm{~s}$ ). This confirms that one of the sources of systematic errors could be continuum determination and measurement of $E W \mathrm{~s}$. In addition, as mentioned above, systematic differences due to the adopted spectral analysis package are present. Indeed, we carried out the analysis of star c6 with MOOG using the $E W \mathrm{~s}$ as measured by $\mathrm{B} 06 \mathrm{a}$ and find a result almost identical to ours, that is, $[\mathrm{Fe} / \mathrm{H}]=+0.02, T_{\text {eff }}=5000 \mathrm{~K}, \log g=2.5$, $\xi=1.18 \mathrm{~km} \mathrm{~s}^{-1}$. All these issues, together with random differences in the final stellar parameters can explain the offset between the two analyses. The average difference $(+0.07$ dex $)$ is compatible within the error bars. Note that, although the metallicities of B06 are in the three cases lower than ours, this is not a completely systematic offset. A random contribution is also present, reflected in the fact that the differences between our results and B06a's change from one star to the other.

In Sect. 5 we mentioned the good agreement between the photometric $T_{\text {eff }}$, assumed as initial parameters, and the final $T_{\text {eff }}$ derived during the spectroscopic analysis. The only star for which we found a rather significant discrepancy is c6, where the $T_{\text {eff }}$ optimized during the analysis is $170 \mathrm{~K}$ cooler than the initial one. We think we can safely assume that the spectroscopic temperature is the right one for two reasons: first, our value is in good agreement with that of B06a. Second, we compared the spectrum of star c6 with those of the other clump objects with similar parameters, and the spectral features confirmed the results. The reason for the wrong photometry of star c6 can be ascribed to the fact that the cluster is affected by differential reddening, as found by Prisinzano et al. (2004) and B06a. That the photometry leads to a hotter $T_{\text {eff }}$ than the real one (which we assume to be the spectroscopic one) could imply that star c6 is not affected by $E(B-V)=0.29$, but by a lower reddening. This agrees with the findings of B06a, which re-derived the reddening for the three stars in their sample from the spectroscopic $T_{\text {eff }}$. In this way, star c6 turned out to have $E(B-V)=0.22$ : assuming a reddening difference of 0.07 dex justifies an error of $\sim 150 \mathrm{~K}$ in $T_{\text {eff }}$.

\subsection{Metallicity distribution in the disk}

How do our results contribute to the picture of the overall metallicity distribution with Galactocentric distance? As already
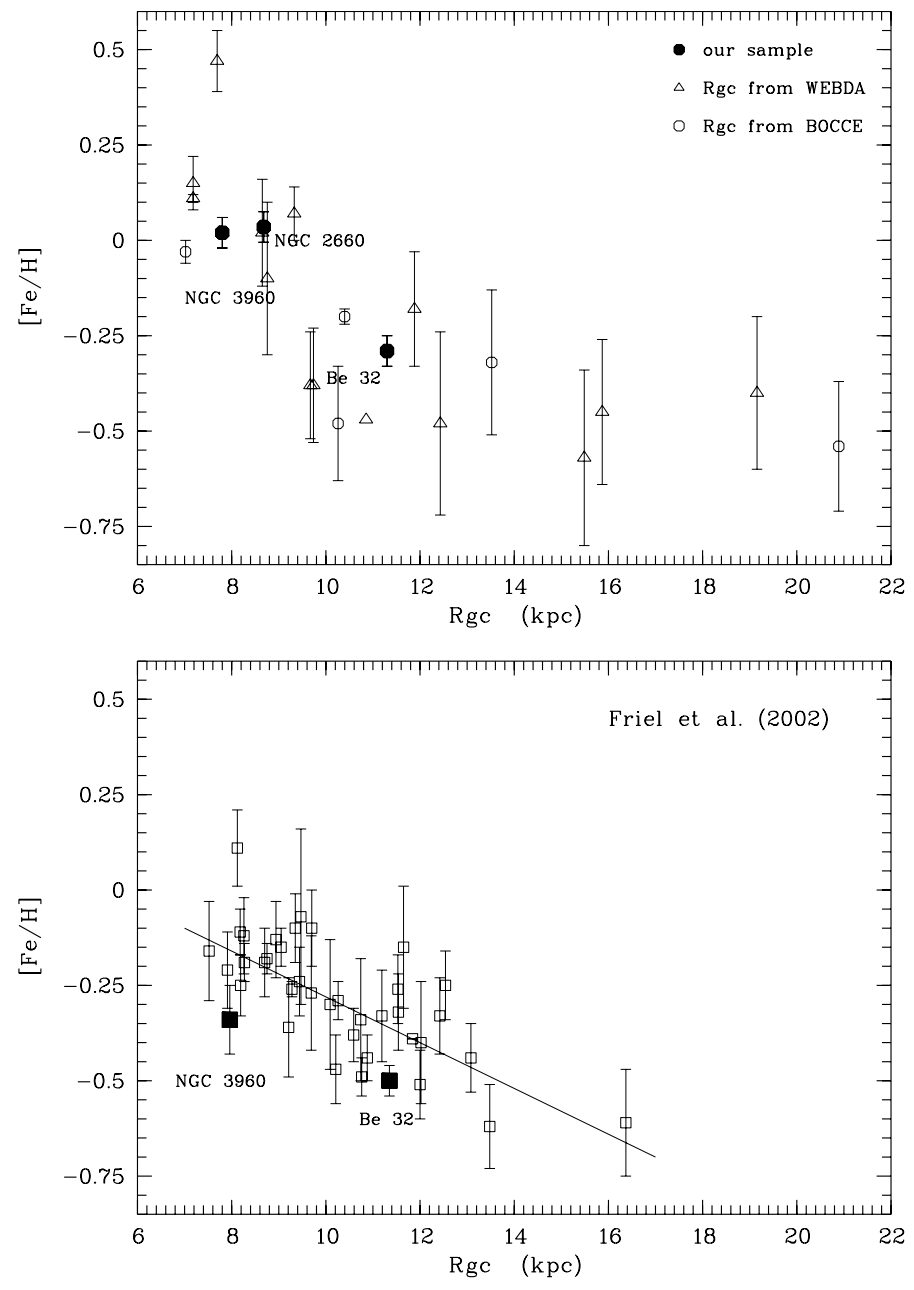

Fig. 7. Radial gradient ([Fe/H] vs. Galactocentric distance) for open clusters. In the upper panel our results (filled circles) are compared to other clusters analyzed with high-resolution spectroscopy (open circles and triangles). In the lower panel the sample analyzed by Friel et al. (2002) is shown (filled symbols are clusters in common with the present work).

mentioned in Sect. 1, a proper comparison should be carried out taking into account the clusters analyzed in a homogeneous way. However, large samples of clusters observed with highresolution spectroscopy and analyzed with the same method are not available yet in the literature; therefore, we report in Fig. 7 plots of $[\mathrm{Fe} / \mathrm{H}]$ as a function Galactocentric distance based on two different datasets. In the upper panel we show our data and the results from other high-resolution studies in the literature $^{6}$. Clusters represented by open circles are included in the BOCCE program (Bragaglia \& Tosi 2006), so we assumed the ages and $R_{\mathrm{gc}}$ listed there. For the three clusters investigated in this work, we assumed the $R_{\mathrm{gc}}$ and ages reported in Table 1. For the remaining clusters we instead assumed ages and $R_{\mathrm{gc}}$ from the WEBDA database ${ }^{7}$. In the lower panel we considered the dataset

${ }^{6}$ Part of these data are collected in Table 7 of Friel et al. (2003, see also references therein); others are from Carretta et al. (2004, 2005), Carraro et al. (2004), Villanova et al. (2005), Yong et al. (2005), Randich et al. (2006), Gratton et al. (2006); note that the quoted investigations are all based on giant stars with the exception of Randich et al. (2006) in which dwarfs and slightly evolved stars of M 67 were considered.

7 http://www. univie.ac.at/webda/ 
by Friel et al. (2002) based on low resolution spectroscopy, which represents a homogeneous sample, as far as the metallicity scale is concerned. Filled symbols are clusters in common with us.

The comparison between the two panels shows fair agreement between the average trends of $[\mathrm{Fe} / \mathrm{H}]$ with $R_{\mathrm{gc}}$ derived from high and low resolution spectra, at least up to distances of $\sim 13 \mathrm{kpc}$ (but see below). Our data are consistent with the existence of a radial gradient, since the clusters at a lower $R_{\mathrm{gc}}$ have higher Fe content. Notice that the spread in the $[\mathrm{Fe} / \mathrm{H}]$ vs. $R_{\mathrm{gc}}$ distribution at low Galactocentric distances appears reduced when using high-resolution data compared the low-resolution ones (excluding NGC 6791 which has $[\mathrm{Fe} / \mathrm{H}]=+0.47$ ). For clusters in the high-resolution sample with $R_{\mathrm{gc}}$ smaller than $\sim 9-10 \mathrm{kpc}$, we find that none has a sub-solar metallicity and that the slope of the $[\mathrm{Fe} / \mathrm{H}]$ vs. Galactocentric radius distribution in the inner $10 \mathrm{kpc}$ is slightly steeper than that derived by Friel et al. at low resolution.

For clusters with $R_{\mathrm{gc}}$ larger than $\sim 13 \mathrm{kpc}$, the Friel dataset does not allow us to distinguish whether the gradient maintains the same slope given by the inner clusters, or it flattens with increasing distance. As previously reported by other authors, the high-resolution data seem to indicate instead that the gradient flattens at large Galactocentric distances.

However, we stress again that this is only an indicative comparison aimed at understanding how our results can be inserted in the average $[\mathrm{Fe} / \mathrm{H}]$ distribution in the Galactic disk.

\section{Summary and conclusions}

We report on the $\mathrm{Fe}$ abundance for three old open clusters, NGC 3960, NGC 2660, and Be 32, based on FLAMES/UVES observations of clump stars.

The data were collected within a VLT/FLAMES program on open clusters and the primary aim was the investigation of the radial metallicity gradient in the Galactic disk, which represents a critical constraint for models of Galactic chemical evolution. Abundances of other elements derived from UVES spectra and the abundances of lithium from the GIRAFFE data of MS stars will be presented in forthcoming papers.

In this paper we accurately describe the method of spectroscopic analysis that will be used for all the clusters in the sample in order to build a homogeneous database with all the abundances and effective temperatures on the same scale. We present the results for the first three clusters analyzed. For the younger clusters NGC 3960 and NGC 2660 (ages $1 \mathrm{Gyr}$ ), we find $[\mathrm{Fe} / \mathrm{H}]=+0.02 \pm 0.04$ (weighted average, $\mathrm{rms}$ ) and $[\mathrm{Fe} / \mathrm{H}]=+0.04 \pm 0.04$, respectively, while the $\sim 6-7$ Gyr old Be 32 turns out to have $[\mathrm{Fe} / \mathrm{H}]=-0.29 \pm 0.04$. We stress that our study represents the first high resolution spectroscopy metallicity investigation for NGC 2660 and Be 32 .

NGC 3960 has been recently investigated by Bragaglia et al. (2006a), who found $[\mathrm{Fe} / \mathrm{H}]=-0.12$ (i.e. a slightly lower value than ours) from high-resolution FEROS data. Previous reports suggested instead a clearly sub-solar $[\mathrm{Fe} / \mathrm{H}]$, at variance with us.

Finally, we discuss our findings in the context of the overall metallicity distribution with Galactocentric radius. With the caveat that the comparison with other (high- and low-resolution spectroscopic) results is biased by the fact that different methods of analysis are used by the various authors, we tentatively conclude that our results support the presence of a negative radial $[\mathrm{Fe} / \mathrm{H}]$ gradient. In addition, the spread in the $[\mathrm{Fe} / \mathrm{H}]$ vs. $R_{\mathrm{gc}}$ distribution appears reduced when using high-resolution data and compared to low-resolution ones. These results should be confirmed based on a larger sample of data analyzed with a homogeneous method.

Acknowledgements. P.S. acknowledges support by the Italian MIUR, under PRIN 2003029437, and of the Bologna Observatory, where this work was completed. We thank the anonymous referee for his/her competent and valuable comments and suggestions to improve the paper. We warmly thank C. Sneden for having provided a new version of MOOG with updates regarding damping parameters. We are grateful to R. Gratton for helpful discussions, and we acknowledge the use of the WEBDA database created by J.-C. Mermilliod.

\section{References}

Allende Prieto, C., Asplund, M., \& Fabiani Bendicho, P. 2004, A\&A, 423, 1109 Alonso, A., Arribas, S., \& Martínez-Roger, C. 1999, A\&AS, 140, 261

Asplund, M., Grevesse, N., \& Sauval, A. J. 2005, in Cosmic Abundances as Records of Stellar Evolution and Nucleosynthesis, ed. T. G. Barnes, \& F. N. Bash, ASP Conf. Ser., 336, 25

Bard, A., \& Kock, M. 1994, A\&A, 282, 1014

Bard, A., Kock, A., \& Kock, M. 1991, A\&A, 248, 315

Barklem, P. S., Piskunov, N., \& O’Mara, B. J. 2000, A\&A, 363, 1091

Beckers, J. M., Bridges, C. A., \& Gilliam, L. B. 1976, A High-Resolution Spectral Atlas of the Solar Irradiance from $\lambda 380$ to $\lambda 700 \mathrm{~nm}$, Sacramento Peak Observatories

Blackwell, D. E., Booth, A. J., Haddock, D. J., Petford, A. D., \& Leggett, S. K. 1986, MNRAS, 220, 549

Boesgaard, A. M., \& Friel, E. D. 1990, ApJ, 351, 467

Boesgaard, A. M., Beard, J. L., \& King, J. R. 2002, AAS, 201.4401, Vol. 34, 1170

Boissier, S., \& Prantzos, N. 1999, MNRAS, 307, 857

Bragaglia, A., \& Tosi, M. 2006, AJ, 131, 1544

Bragaglia, A., Carretta, E., Gratton, R., et al. 2001, AJ, 121, 327

Bragaglia, A., Tosi, M., Carretta, E., et al. 2006a, MNRAS, 366, 1493 (B06a)

Bertelli, G., Bressan, A., Chiosi, C., Fagotto, F., \& Nasi, E. 1994, A\&AS, 106, 275

Carraro, G., Ng, Y. K., \& Portinari, L. 1998, MNRAS, 296, 1045

Carraro, G., Bresolin, F., Villanova, S., et al. 2004, AJ, 128, 1676

Carraro, G., Villanova, S., Demarque, P., et al. 2006, AJ, 643, 1151

Carretta, E., Bragaglia, A., Gratton, R., \& Tosi, M. 2004, A\&A, 422, 951

Carretta, E., Bragaglia, A., Gratton, R., \& Tosi, M. 2005, A\&A, 441, 131

Chiappini, C., Matteucci, F., \& Gratton, R. 1997, ApJ, 477, 765

Corder, S., \& Twarog, B. A. 2001, AJ, 122, 895

Cutri, R. M., Skrutskie, M. F., van Dyk, S., et al. 2003, VizieR On-line Data Catalog, II/246, University of Massachusetts and IPA/California Institute of Technology

Delbouille, L., Roland, G., \& Neven, L. 1973, Atlas photometrique du spectre solaire de $\lambda 3000$ a $\lambda 10000$, Université de Liege, Institut d'Astrophysique

D’Orazi, V., Bragaglia, A., Tosi, M., \& Held, E. 2006, MNRAS, 368, 471

Edvardsson, B., Andersen, J., Gustafsson, B., et al. 1993, A\&AS, 102, 603

Feltzing, S., \& Gustafsson, B. 1998, A\&AS, 129, 237

Fitzpatrick, M. J., \& Sneden, C. 1987, BAAS, 19, 1129

Friel, E. D. 1995, ARA\&A, 33, 381

Friel, E. D. 2006, in Chemical Abundances and Mixing in Stars in the Milky Way and its Satellites, ed. L. Pasquini, \& S. Randich, ESO Astrophys. Symp., 24, 3

Friel, E. D., \& Janes, K. A. 1993, A\&A, 267, 75

Friel, E. D., Janes, K. A., Tavarez, M., et al. 2002, AJ, 124, 2693

Friel, E. D., Jacobson, H. R., Barrett, E., et al. 2003, AJ, 126, 2372

Geisler, D., Clarià, J. J., \& Minniti, D. 1992, AJ, 104, 1892

Giovagnoli, A., \& Tosi, M. 1995, MNRAS, 273, 499

Gratton, R. 1988, Rome Obs. Preprint Ser., 29

Gratton, R., Carretta, E., Claudi, R., Lucatello, S., \& Barbieri, M. 2003, A\&A, 404, 187 (G03)

Gratton, R., Bragaglia, A., Carretta, E., \& Tosi, M. 2006, ApJ, 642, 462

Gray, D. F. 1976, Observations and analysis of stellar photospheres (Cambridge University Press)

Grevesse, N., \& Sauval, A. J. 1999, A\&A, 347, 348

Hartwick, F. D., \& Hesser, J. E. 1973, ApJ, 183, 883

Hasegawa, T., Malasan, H. M., Kawakita, H., et al. 2004, PASJ, 56, 295

Hesser, J. E., \& Smith, G. H. 1987, PASP, 309, 1044

Janes, K. A. 1981, AJ, 86, 1210

Kaluzny, J., \& Mazur, B. 1991, Acta Astron., 41, 167

Kurucz, R. L. 1993, CD-ROM No. 9 
Kurucz, R. L., Furenlid, I., Brault, J., \& Testerman, L. 1984, Solar Flux Atlas from 296 to $1300 \mathrm{~nm}$, NOAO Atlas No. 1

Lacey, C. G., \& Fall, S. M. 1985, ApJ, 290, 154

Maciel, W. J., Costa, R. D. D., \& Uchida, M. M. M. 2003, A\&A, 397, 667

Magain, P. 1984, A\&A, 134, 189

Mermilliod, J.-C., Clarià, J. J., Andersen, J., Piatti, A. E., \& Mayor, M. 2001, A\&A, 375, 30

Mulas, G., Modigliani, A., Porceddu, I., \& Damiani, F. 2002, SPIE, 4844, 310

Noriega-Mendoza, H., \& Ruelas-Mayorga, A. 1997, AJ, 113, 722

O'Brian, T. R., Wickliffe, M. E., Lawler, J. E., Whaling, J. W., \& Brault, W. 1991, JOSA B, 8, 1185

Pasquali, A., \& Perinotto, M. 1993, A\&A, 280, 581

Pasquini, L., Avila, G., Allaert, E., et al. 2000, SPIE, 4008, 129

Paulson, D. B., Sneden, C., \& Cochran, W. D. 2003, AJ, 125, 3185

Piatti, A. E., Clarià, J. J., \& Abadi, M. G. 1995, AJ, 309, 2813

Prisinzano, L., Micela, G., Sciortino, S., \& Favata, F. 2004, A\&A, 417, 945

Randich, S., Bragaglia, A., Pastori, L., et al. 2005, ESO Messenger, 121, 18

Randich, S., Sestito, P., Primas, F., Pasquini, L., \& Pallavicini, R. 2006, A\&A, 450,557
Richtler, T., \& Sagar, R. 2001, BASI, 29, 53

Rutten, R. J., \& Van der Zalm, E. B. J. 1984, A\&AS, 55, 171

Sandrelli, S., Bragaglia, A., Tosi, M., \& Marconi, G. 1999, MNRAS, 309, 739

Schuler, S. C., King, J. R., Fischer, D. A., Soderblom, D. R., \& Jones, B. F. 2003, AJ, 125, 2085

Schuler, S. C., Hatzes, A. P., King, J. R., Kuerster, M., \& The, L.-S. 2006, AJ, 131,1057

Shaver, P. A., McGee, R. X., Newton, L. M., Danks, A. C., \& Pottasch, S. R. 1983, MNRAS, 204, 53

Smartt, S. J., \& Rollerstone, W. R. J. 1997, ApJ, 4181, L47

Smith, G. 1999, A\&A, 350, 859

Sneden, C. A. 1973, ApJ, 184, 839

Tosi, M. 1988, A\&A, 197, 33

Tosi, M. 1996, ASP Conf. Ser., 98, 299

Twarog, B. A., Ashman, K. M., \& Anthony-Twarog, B. J. 1997, AJ, 114, 2556

Unsöld, A. 1955, Physik der Sternatmosphären (Berlin: Springer-Verlag)

Villanova, S., Carraro, G., Bresolin, F., \& Patat, F. 2005, AJ, 130, 652

Wylie, E. C., Cottrell, P. L., \& Taute, K. M. 2004, MemSAIt, 75, 578

Yong, D., Carney, B. W., \& de Almeida, L. 2005, AJ, 130, 597 
P. Sestito et al.: Chemical abundances in open clusters. I., Online Material $p 1$

\section{Online Material}


P. Sestito et al.: Chemical abundances in open clusters. I., Online Material p 2

Table 7. Fe line list for the Sun and equivalent widths.

\begin{tabular}{|c|c|c|c|c|c|}
\hline $\begin{array}{l}\text { Wavelength } \\
(\AA)\end{array}$ & Atom & $\begin{array}{l}E P \\
\mathrm{eV}\end{array}$ & $\log g f$ & $\begin{array}{l}E W \\
(\mathrm{~m} \AA)\end{array}$ & $\begin{array}{l}\text { damping } \\
\text { (ref.) }\end{array}$ \\
\hline 4389.25 & Fe I & 0.052 & -4.58 & 71.7 & 2 \\
\hline 4439.89 & $\mathrm{Fe} \mathrm{I}$ & 2.279 & -3.00 & 50.0 & 2 \\
\hline 4442.84 & $\mathrm{Fe} \mathrm{I}$ & 2.176 & -2.79 & 63.0 & 2 \\
\hline 4445.48 & $\mathrm{Fe} \mathrm{I}$ & 0.087 & -5.44 & 38.8 & 3 \\
\hline 4523.41 & $\mathrm{Fe} \mathrm{I}$ & 3.654 & -1.92 & 42.9 & 2 \\
\hline 4551.65 & $\mathrm{Fe} \mathrm{I}$ & 3.944 & -2.05 & 25.0 & 2 \\
\hline 4556.93 & $\mathrm{Fe} \mathrm{I}$ & 3.252 & -2.64 & 26.4 & 3 \\
\hline 4574.23 & $\mathrm{Fe} \mathrm{I}$ & 3.211 & -2.38 & 39.8 & 2 \\
\hline 4593.53 & $\mathrm{Fe} \mathrm{I}$ & 3.944 & -1.97 & 28.0 & 2 \\
\hline 4658.30 & $\mathrm{Fe} \mathrm{I}$ & 3.267 & -2.96 & 14.5 & 2 \\
\hline 4672.84 & $\mathrm{Fe} \mathrm{I}$ & 1.608 & -4.04 & 33.2 & 2 \\
\hline 4726.15 & $\mathrm{Fe} \mathrm{I}$ & 2.998 & -3.18 & 15.3 & 2 \\
\hline 4745.14 & $\mathrm{Fe} \mathrm{I}$ & 2.223 & -4.12 & 10.4 & 2 \\
\hline 4779.45 & $\mathrm{Fe} \mathrm{I}$ & 3.415 & -2.28 & 40.2 & 2 \\
\hline 4788.76 & $\mathrm{Fe} \mathrm{I}$ & 3.241 & -1.70 & 70.8 & 2 \\
\hline 4794.36 & $\mathrm{Fe} \mathrm{I}$ & 2.424 & -3.91 & 10.8 & 2 \\
\hline 4798.27 & $\mathrm{Fe} \mathrm{I}$ & 4.187 & -1.46 & 41.8 & 3 \\
\hline 4802.52 & $\mathrm{Fe} \mathrm{I}$ & 4.607 & -1.73 & 14.6 & 2 \\
\hline 4808.16 & $\mathrm{Fe} \mathrm{I}$ & 3.252 & -2.64 & 26.3 & 2 \\
\hline 4809.94 & $\mathrm{Fe} \mathrm{I}$ & 3.573 & -2.55 & 18.0 & 2 \\
\hline 4835.87 & $\mathrm{Fe} \mathrm{I}$ & 4.104 & -1.43 & 47.3 & 2 \\
\hline 4885.43 & $\mathrm{Fe} \mathrm{I}$ & 3.882 & -1.10 & 72.6 & 3 \\
\hline 4892.87 & $\mathrm{Fe} \mathrm{I}$ & 4.218 & -1.34 & 52.4 & 2 \\
\hline 4905.14 & $\mathrm{Fe} \mathrm{I}$ & 3.929 & -1.91 & 31.2 & 2 \\
\hline 4917.24 & $\mathrm{Fe} \mathrm{I}$ & 4.191 & -1.29 & 51.1 & 2 \\
\hline 4961.92 & $\mathrm{Fe} \mathrm{I}$ & 3.635 & -2.34 & 23.6 & 2 \\
\hline 4962.58 & $\mathrm{Fe} \mathrm{I}$ & 4.178 & -1.20 & 56.7 & 3 \\
\hline 5016.48 & $\mathrm{Fe} \mathrm{I}$ & 4.257 & -1.62 & 30.8 & 2 \\
\hline 5058.49 & $\mathrm{Fe} I$ & 3.642 & -2.76 & 11.0 & 2 \\
\hline 5141.75 & $\mathrm{Fe} \mathrm{I}$ & 2.424 & -1.96 & 94.4 & 2 \\
\hline 5187.92 & $\mathrm{Fe} \mathrm{I}$ & 4.143 & -1.26 & 55.8 & 3 \\
\hline 5196.06 & $\mathrm{Fe} \mathrm{I}$ & 4.257 & -0.82 & 75.6 & 3 \\
\hline 5217.40 & $\mathrm{Fe} \mathrm{I}$ & 3.211 & -1.07 & 122.0 & 2 \\
\hline 5223.19 & $\mathrm{Fe} \mathrm{I}$ & 3.635 & -2.28 & 27.4 & 2 \\
\hline 5225.53 & $\mathrm{Fe} \mathrm{I}$ & 0.110 & -4.79 & 71.0 & 2 \\
\hline 5243.78 & $\mathrm{Fe} \mathrm{I}$ & 4.257 & -1.04 & 62.3 & 2 \\
\hline 5247.06 & $\mathrm{Fe} \mathrm{I}$ & 0.087 & -4.95 & 65.8 & 2 \\
\hline 5250.22 & $\mathrm{Fe} I$ & 0.121 & -4.94 & 64.9 & 2 \\
\hline 5253.03 & $\mathrm{Fe} \mathrm{I}$ & 2.279 & -3.84 & 16.8 & 2 \\
\hline 5294.55 & $\mathrm{Fe} \mathrm{I}$ & 3.640 & -2.70 & 12.7 & 2 \\
\hline 5295.32 & $\mathrm{Fe} \mathrm{I}$ & 4.416 & -1.55 & 28.2 & 2 \\
\hline 5320.04 & $\mathrm{Fe} \mathrm{I}$ & 3.642 & -2.54 & 17.5 & 3 \\
\hline 5321.11 & $\mathrm{Fe} \mathrm{I}$ & 4.435 & -1.25 & 43.0 & 2 \\
\hline 5324.19 & $\mathrm{Fe} \mathrm{I}$ & 3.211 & -0.10 & 305.0 & 2 \\
\hline 5326.82 & $\mathrm{Fe} \mathrm{I}$ & 4.416 & -2.09 & 11.8 & 2 \\
\hline 5376.84 & $\mathrm{Fe} \mathrm{I}$ & 4.295 & -2.07 & 13.6 & 2 \\
\hline 5379.58 & $\mathrm{Fe} \mathrm{I}$ & 3.695 & -1.65 & 63.6 & 2 \\
\hline 5386.34 & $\mathrm{Fe} \mathrm{I}$ & 4.155 & -1.74 & 30.9 & 2 \\
\hline 5389.49 & $\mathrm{Fe} \mathrm{I}$ & 4.416 & -0.57 & 86.0 & 2 \\
\hline 5393.18 & $\mathrm{Fe} \mathrm{I}$ & 3.241 & -0.72 & 145.0 & 2 \\
\hline 5395.22 & $\mathrm{Fe} \mathrm{I}$ & 4.446 & -1.73 & 19.4 & 2 \\
\hline 5398.29 & $\mathrm{Fe} \mathrm{I}$ & 4.446 & -0.72 & 74.0 & 2 \\
\hline 5401.27 & $\mathrm{Fe} \mathrm{I}$ & 4.320 & -1.74 & 22.8 & 2 \\
\hline 5405.78 & $\mathrm{Fe} I$ & 0.990 & -1.84 & 265.0 & 2 \\
\hline 5406.78 & $\mathrm{Fe} \mathrm{I}$ & 4.372 & -1.40 & 37.6 & 2 \\
\hline 5412.79 & $\mathrm{Fe} \mathrm{I}$ & 4.435 & -1.72 & 17.8 & 2 \\
\hline 5417.04 & $\mathrm{Fe} I$ & 4.416 & -1.42 & 34.9 & 2 \\
\hline 5434.53 & $\mathrm{Fe} \mathrm{I}$ & 1.011 & -2.12 & 190.0 & 2 \\
\hline 5436.30 & $\mathrm{Fe} \mathrm{I}$ & 4.387 & -1.36 & 38.9 & 2 \\
\hline 5464.29 & $\mathrm{Fe} \mathrm{I}$ & 4.143 & -1.62 & 37.3 & 3 \\
\hline 5470.09 & Fe I & 4.446 & -1.60 & 25.5 & 2 \\
\hline 5494.47 & $\mathrm{Fe} \mathrm{I}$ & 4.076 & -1.96 & 24.8 & 1 \\
\hline 5522.45 & $\mathrm{Fe} \mathrm{I}$ & 4.209 & -1.47 & 42.4 & 2 \\
\hline
\end{tabular}

Table 7. continued.

\begin{tabular}{|c|c|c|c|c|c|}
\hline $\begin{array}{l}\text { Wavelength } \\
(\AA)\end{array}$ & Atom & $\begin{array}{l}E P \\
\mathrm{eV}\end{array}$ & $\log g f$ & $\begin{array}{l}E W \\
(\mathrm{~m} \AA)\end{array}$ & $\begin{array}{l}\text { damping } \\
\text { (ref.) }\end{array}$ \\
\hline 5539.29 & Fe I & 3.642 & -2.59 & 16.5 & 2 \\
\hline 5547.00 & $\mathrm{Fe} I$ & 4.218 & -1.85 & 23.9 & 2 \\
\hline 5560.22 & $\mathrm{Fe} \mathrm{I}$ & 4.435 & -1.10 & 51.8 & 2 \\
\hline 5586.77 & $\mathrm{Fe} \mathrm{I}$ & 3.369 & -0.10 & 220.0 & 2 \\
\hline 5587.58 & $\mathrm{Fe} \mathrm{I}$ & 4.143 & -1.70 & 33.5 & 1 \\
\hline 5618.64 & $\mathrm{Fe} \mathrm{I}$ & 4.209 & -1.34 & 49.3 & 2 \\
\hline 5619.61 & $\mathrm{Fe} \mathrm{I}$ & 4.387 & -1.49 & 34.9 & 2 \\
\hline 5635.83 & $\mathrm{Fe} \mathrm{I}$ & 4.256 & -1.59 & 33.7 & 2 \\
\hline 5636.70 & $\mathrm{Fe} \mathrm{I}$ & 3.640 & -2.53 & 18.2 & 2 \\
\hline 5650.00 & $\mathrm{Fe} \mathrm{I}$ & 5.086 & -0.80 & 37.5 & 2 \\
\hline 5651.48 & $\mathrm{Fe} \mathrm{I}$ & 4.474 & -1.79 & 17.0 & 2 \\
\hline 5652.33 & $\mathrm{Fe} \mathrm{I}$ & 4.261 & -1.77 & 25.5 & 2 \\
\hline 5661.35 & $\mathrm{Fe} \mathrm{I}$ & 4.285 & -1.83 & 19.8 & 2 \\
\hline 5701.56 & $\mathrm{Fe} \mathrm{I}$ & 2.559 & -2.16 & 86.0 & 2 \\
\hline 5717.84 & $\mathrm{Fe} \mathrm{I}$ & 4.285 & -0.98 & 67.0 & 2 \\
\hline 5731.77 & $\mathrm{Fe} \mathrm{I}$ & 4.256 & -1.10 & 60.7 & 2 \\
\hline 5738.24 & $\mathrm{Fe} \mathrm{I}$ & 4.221 & -2.24 & 11.7 & 2 \\
\hline 5741.86 & $\mathrm{Fe} \mathrm{I}$ & 4.256 & -1.69 & 30.6 & 2 \\
\hline 5752.04 & $\mathrm{Fe} \mathrm{I}$ & 4.549 & -0.92 & 57.5 & 1 \\
\hline 5760.36 & $\mathrm{Fe} \mathrm{I}$ & 3.642 & -2.46 & 20.9 & 2 \\
\hline 5775.09 & $\mathrm{Fe} \mathrm{I}$ & 4.221 & -1.11 & 62.3 & 1 \\
\hline 5778.46 & $\mathrm{Fe} \mathrm{I}$ & 2.588 & -3.44 & 19.5 & 2 \\
\hline 5784.67 & $\mathrm{Fe} I$ & 3.397 & -2.53 & 25.0 & 2 \\
\hline 5793.92 & $\mathrm{Fe} \mathrm{I}$ & 4.221 & -1.62 & 34.8 & 2 \\
\hline 5806.73 & $\mathrm{Fe} \mathrm{I}$ & 4.608 & -0.93 & 53.4 & 2 \\
\hline 5814.81 & $\mathrm{Fe} \mathrm{I}$ & 4.284 & -1.81 & 22.4 & 2 \\
\hline 5835.11 & $\mathrm{Fe} \mathrm{I}$ & 4.257 & -2.18 & 12.1 & 2 \\
\hline 5852.23 & $\mathrm{Fe} \mathrm{I}$ & 4.549 & -1.36 & 41.7 & 2 \\
\hline 5855.09 & $\mathrm{Fe} \mathrm{I}$ & 4.608 & -1.56 & 21.0 & 2 \\
\hline 5856.10 & $\mathrm{Fe} \mathrm{I}$ & 4.295 & -1.57 & 33.9 & 2 \\
\hline 5858.78 & $\mathrm{Fe} \mathrm{I}$ & 4.221 & -2.19 & 12.8 & 2 \\
\hline 5859.60 & $\mathrm{Fe} \mathrm{I}$ & 4.549 & -0.63 & 75.3 & 1 \\
\hline 5862.37 & $\mathrm{Fe} \mathrm{I}$ & 4.549 & -0.42 & 90.8 & 1 \\
\hline 5902.48 & $\mathrm{Fe} \mathrm{I}$ & 4.593 & -1.86 & 12.7 & 2 \\
\hline 5905.68 & $\mathrm{Fe} \mathrm{I}$ & 4.652 & -0.78 & 60.9 & 2 \\
\hline 5927.80 & $\mathrm{Fe} \mathrm{I}$ & 4.652 & -1.07 & 43.6 & 2 \\
\hline 5929.68 & $\mathrm{Fe} \mathrm{I}$ & 4.549 & -1.16 & 38.7 & 2 \\
\hline 5930.19 & $\mathrm{Fe} \mathrm{I}$ & 4.652 & -0.34 & 92.7 & 2 \\
\hline 5934.67 & $\mathrm{Fe} \mathrm{I}$ & 3.929 & -1.08 & 79.0 & 2 \\
\hline 5956.71 & $\mathrm{Fe} \mathrm{I}$ & 0.859 & -4.56 & 50.8 & 2 \\
\hline 5976.79 & $\mathrm{Fe} I$ & 3.944 & -1.30 & 65.4 & 1 \\
\hline 5984.83 & $\mathrm{Fe} \mathrm{I}$ & 4.733 & -0.29 & 91.8 & 1 \\
\hline 6003.02 & $\mathrm{Fe} \mathrm{I}$ & 3.882 & -1.02 & 85.4 & 2 \\
\hline 6007.97 & $\mathrm{Fe} \mathrm{I}$ & 4.652 & -0.76 & 62.2 & 1 \\
\hline 6008.57 & $\mathrm{Fe} \mathrm{I}$ & 3.884 & -0.92 & 91.8 & 1 \\
\hline 6027.06 & $\mathrm{Fe} \mathrm{I}$ & 4.076 & -1.20 & 64.9 & 1 \\
\hline 6056.01 & $\mathrm{Fe} \mathrm{I}$ & 4.733 & -0.46 & 78.0 & 2 \\
\hline 6065.49 & $\mathrm{Fe} \mathrm{I}$ & 2.609 & -1.49 & 118.8 & 2 \\
\hline 6078.50 & $\mathrm{Fe} \mathrm{I}$ & 4.796 & -0.38 & 80.7 & 1 \\
\hline 6079.02 & $\mathrm{Fe} \mathrm{I}$ & 4.652 & -0.97 & 49.2 & 2 \\
\hline 6082.72 & $\mathrm{Fe} \mathrm{I}$ & 2.223 & -3.53 & 34.0 & 2 \\
\hline 6089.57 & $\mathrm{Fe} \mathrm{I}$ & 4.580 & -0.87 & 36.9 & 1 \\
\hline 6093.65 & $\mathrm{Fe} \mathrm{I}$ & 4.608 & -1.32 & 29.5 & 2 \\
\hline 6094.38 & $\mathrm{Fe} I$ & 4.652 & -1.56 & 19.6 & 2 \\
\hline 6096.67 & $\mathrm{Fe} I$ & 3.984 & -1.76 & 38.7 & 2 \\
\hline 6098.25 & $\mathrm{Fe} \mathrm{I}$ & 4.559 & -1.81 & 14.5 & 2 \\
\hline 6137.00 & $\mathrm{Fe} \mathrm{I}$ & 2.198 & -2.91 & 63.8 & 2 \\
\hline 6151.62 & $\mathrm{Fe} I$ & 2.176 & -3.26 & 46.9 & 2 \\
\hline 6157.73 & $\mathrm{Fe} \mathrm{I}$ & 4.076 & -1.26 & 61.9 & 1 \\
\hline 6165.36 & $\mathrm{Fe} \mathrm{I}$ & 4.143 & -1.48 & 46.2 & 1 \\
\hline 6173.34 & $\mathrm{Fe} \mathrm{I}$ & 2.223 & -2.84 & 67.4 & 2 \\
\hline 6188.00 & $\mathrm{Fe} \mathrm{I}$ & 3.944 & -1.60 & 49.5 & 2 \\
\hline 6200.32 & Fe I & 2.609 & -2.39 & 75.6 & 2 \\
\hline
\end{tabular}


P. Sestito et al.: Chemical abundances in open clusters. I., Online Material p 3

Table 7. continued.

\begin{tabular}{|c|c|c|c|c|c|}
\hline $\begin{array}{l}\text { Wavelength } \\
(\AA) \\
(\AA)\end{array}$ & Atom & $\begin{array}{l}E P \\
\mathrm{eV}\end{array}$ & $\overline{\log g f}$ & $\begin{array}{l}E W \\
(\mathrm{~m} \AA)\end{array}$ & $\begin{array}{l}\text { damping } \\
\text { (ref.) }\end{array}$ \\
\hline 6213.44 & Fe I & 2.223 & -2.54 & 82.7 & 2 \\
\hline 6219.29 & $\mathrm{Fe} \mathrm{I}$ & 2.198 & -2.43 & 89.2 & 2 \\
\hline 6220.79 & $\mathrm{Fe} \mathrm{I}$ & 3.882 & -2.36 & 17.1 & 2 \\
\hline 6226.74 & $\mathrm{Fe} \mathrm{I}$ & 3.882 & -2.08 & 28.6 & 2 \\
\hline 6232.65 & $\mathrm{Fe} \mathrm{I}$ & 3.654 & -1.21 & 87.0 & 3 \\
\hline 6240.65 & $\mathrm{Fe} \mathrm{I}$ & 2.223 & -3.23 & 43.8 & 2 \\
\hline 6246.33 & $\mathrm{Fe} \mathrm{I}$ & 3.603 & -0.73 & 127.0 & 2 \\
\hline 6252.56 & $\mathrm{Fe} \mathrm{I}$ & 2.404 & -1.64 & 122.8 & 2 \\
\hline 6265.14 & $\mathrm{Fe} \mathrm{I}$ & 2.176 & -2.51 & 86.8 & 2 \\
\hline 6270.23 & $\mathrm{Fe} \mathrm{I}$ & 2.858 & -2.46 & 52.3 & 2 \\
\hline 6280.62 & $\mathrm{Fe} \mathrm{I}$ & 0.859 & -4.34 & 62.4 & 2 \\
\hline 6297.80 & $\mathrm{Fe} \mathrm{I}$ & 2.223 & -2.70 & 74.2 & 2 \\
\hline 6301.51 & $\mathrm{Fe} \mathrm{I}$ & 3.654 & -0.72 & 132.0 & 3 \\
\hline 6311.50 & $\mathrm{Fe} \mathrm{I}$ & 2.832 & -3.16 & 26.1 & 2 \\
\hline 6315.81 & $\mathrm{Fe} \mathrm{I}$ & 4.076 & -1.67 & 40.0 & 1 \\
\hline 6322.69 & $\mathrm{Fe} \mathrm{I}$ & 2.588 & -2.38 & 79.2 & 2 \\
\hline 6330.85 & $\mathrm{Fe} \mathrm{I}$ & 4.733 & -1.22 & 32.5 & 2 \\
\hline 6335.34 & $\mathrm{Fe} \mathrm{I}$ & 2.198 & -2.28 & 98.8 & 2 \\
\hline 6380.75 & $\mathrm{Fe} \mathrm{I}$ & 4.187 & -1.34 & 52.2 & 1 \\
\hline 6392.54 & $\mathrm{Fe} \mathrm{I}$ & 2.279 & -3.97 & 15.2 & 2 \\
\hline 6393.61 & $\mathrm{Fe} \mathrm{I}$ & 2.433 & -1.43 & 139.0 & 2 \\
\hline 6411.66 & $\mathrm{Fe} \mathrm{I}$ & 3.654 & -0.60 & 143.0 & 2 \\
\hline 6421.36 & $\mathrm{Fe} \mathrm{I}$ & 2.279 & -1.98 & 110.0 & 2 \\
\hline 6481.88 & $\mathrm{Fe} \mathrm{I}$ & 2.279 & -2.94 & 63.6 & 2 \\
\hline 6498.94 & $\mathrm{Fe} \mathrm{I}$ & 0.958 & -4.66 & 44.3 & 2 \\
\hline 6518.37 & $\mathrm{Fe} \mathrm{I}$ & 2.832 & -2.56 & 54.6 & 2 \\
\hline 6533.94 & $\mathrm{Fe} \mathrm{I}$ & 4.559 & -1.28 & 37.7 & 2 \\
\hline 6574.25 & $\mathrm{Fe} \mathrm{I}$ & 0.990 & -5.00 & 26.5 & 1 \\
\hline 6581.22 & $\mathrm{Fe} \mathrm{I}$ & 1.485 & -4.68 & 14.1 & 2 \\
\hline 6593.88 & $\mathrm{Fe} \mathrm{I}$ & 2.433 & -2.30 & 86.4 & 2 \\
\hline 6608.04 & $\mathrm{Fe} \mathrm{I}$ & 2.279 & -3.96 & 15.9 & 2 \\
\hline 6609.12 & $\mathrm{Fe} \mathrm{I}$ & 2.559 & -2.65 & 65.5 & 2 \\
\hline 6625.04 & $\mathrm{Fe} \mathrm{I}$ & 1.011 & -5.32 & 14.5 & 1 \\
\hline 6627.56 & $\mathrm{Fe} \mathrm{I}$ & 4.549 & -1.50 & 26.7 & 2 \\
\hline 6633.76 & $\mathrm{Fe} \mathrm{I}$ & 4.559 & -0.81 & 64.7 & 2 \\
\hline 6703.58 & $\mathrm{Fe} \mathrm{I}$ & 2.759 & -3.00 & 36.6 & 2 \\
\hline 6713.75 & $\mathrm{Fe} \mathrm{I}$ & 4.796 & -1.41 & 21.2 & 2 \\
\hline 6725.36 & $\mathrm{Fe} \mathrm{I}$ & 4.104 & -2.21 & 16.5 & 2 \\
\hline 6726.67 & $\mathrm{Fe} \mathrm{I}$ & 4.607 & -1.05 & 48.5 & 1 \\
\hline 6733.15 & $\mathrm{Fe} \mathrm{I}$ & 4.639 & -1.44 & 26.9 & 2 \\
\hline 6750.16 & $\mathrm{Fe} \mathrm{I}$ & 2.424 & -2.58 & 75.8 & 2 \\
\hline 6786.86 & $\mathrm{Fe} \mathrm{I}$ & 4.191 & -1.90 & 24.0 & 2 \\
\hline 6804.30 & $\mathrm{Fe} \mathrm{I}$ & 4.585 & -1.85 & 14.0 & 2 \\
\hline 6806.86 & $\mathrm{Fe} \mathrm{I}$ & 2.728 & -3.14 & 31.5 & 2 \\
\hline 6810.27 & $\mathrm{Fe} \mathrm{I}$ & 4.607 & -1.00 & 51.8 & 2 \\
\hline 6820.37 & $\mathrm{Fe} \mathrm{I}$ & 4.639 & -1.16 & 41.1 & 2 \\
\hline 6837.01 & Fe I & 4.593 & -1.71 & 15.4 & 2 \\
\hline 6839.83 & $\mathrm{Fe} \mathrm{I}$ & 2.559 & -3.35 & 29.2 & 2 \\
\hline 6843.66 & $\mathrm{Fe} \mathrm{I}$ & 4.549 & -0.86 & 63.4 & 2 \\
\hline 6855.72 & $\mathrm{Fe} \mathrm{I}$ & 4.607 & -1.71 & 17.0 & 2 \\
\hline 6857.25 & $\mathrm{Fe} \mathrm{I}$ & 4.076 & -2.07 & 21.9 & 1 \\
\hline 6858.16 & $\mathrm{Fe} \mathrm{I}$ & 4.608 & -0.95 & 54.6 & 2 \\
\hline 6861.94 & $\mathrm{Fe} \mathrm{I}$ & 2.424 & -3.78 & 17.8 & 2 \\
\hline 6862.50 & $\mathrm{Fe} I$ & 4.559 & -1.43 & 30.7 & 2 \\
\hline 4178.86 & $\mathrm{Fe}$ II & 2.583 & -2.538 & 5.0 & 2 \\
\hline 4491.40 & $\mathrm{Fe}$ II & 2.856 & -2.607 & 8.0 & 2 \\
\hline 4508.30 & $\mathrm{Fe}$ II & 2.856 & -2.28 & 89.7 & 2 \\
\hline 4541.52 & $\mathrm{Fe}$ II & 2.856 & -2.81 & 68.3 & 2 \\
\hline 4555.89 & Fe II & 2.828 & -2.18 & 93.9 & 2 \\
\hline 4576.34 & $\mathrm{Fe}$ II & 2.844 & -2.90 & 67.4 & 2 \\
\hline 4582.83 & $\mathrm{Fe}$ II & 2.844 & -3.10 & 58.0 & 2 \\
\hline 4620.52 & Fe II & 2.828 & -3.20 & 54.7 & 2 \\
\hline 4635.31 & Fe II & 5.956 & -1.28 & 19.3 & 2 \\
\hline
\end{tabular}

Table 7. continued.

\begin{tabular}{|c|c|c|c|c|c|}
\hline $\begin{array}{l}\text { Wavelength } \\
(\AA) \\
(\AA)\end{array}$ & Atom & $\begin{array}{l}E P \\
\mathrm{eV}\end{array}$ & $\log g f$ & $\begin{array}{l}E W \\
(\mathrm{~m} \AA)\end{array}$ & $\begin{array}{l}\text { damping } \\
\text { (ref.) }\end{array}$ \\
\hline 4656.98 & Fe II & 2.891 & -3.59 & 37.0 & 2 \\
\hline 4670.17 & $\mathrm{Fe}$ II & 2.583 & -3.97 & 31.8 & 2 \\
\hline 4825.72 & $\mathrm{Fe}$ II & 2.635 & -4.89 & 7.0 & 2 \\
\hline 4833.19 & $\mathrm{Fe}$ II & 2.657 & -4.62 & 11.4 & 2 \\
\hline 4840.00 & $\mathrm{Fe}$ II & 2.676 & -4.74 & 8.8 & 2 \\
\hline 4893.82 & $\mathrm{Fe}$ II & 2.828 & -4.29 & 15.8 & 2 \\
\hline 4993.35 & $\mathrm{Fe}$ II & 2.807 & -3.56 & 39.7 & 2 \\
\hline 5100.66 & $\mathrm{Fe}$ II & 2.807 & -4.16 & 19.4 & 2 \\
\hline 5132.67 & Fe II & 2.807 & -3.95 & 25.5 & 2 \\
\hline 5136.80 & $\mathrm{Fe}$ II & 2.844 & -4.32 & 14.6 & 2 \\
\hline 5197.58 & $\mathrm{Fe}$ II & 3.231 & -2.23 & 85.8 & 2 \\
\hline 5234.63 & $\mathrm{Fe}$ II & 3.221 & -2.22 & 88.9 & 2 \\
\hline 5264.81 & $\mathrm{Fe}$ II & 3.339 & -3.21 & 47.2 & 2 \\
\hline 5284.11 & Fe II & 2.891 & -3.01 & 63.0 & 2 \\
\hline 5325.56 & Fe II & 3.221 & -3.18 & 44.7 & 2 \\
\hline 5414.08 & Fe II & 3.221 & -3.61 & 27.2 & 2 \\
\hline 5425.26 & $\mathrm{Fe}$ II & 3.200 & -3.27 & 44.0 & 2 \\
\hline 5525.13 & $\mathrm{Fe}$ II & 3.267 & -4.04 & 12.9 & 2 \\
\hline 5534.85 & Fe II & 3.245 & -2.75 & 61.3 & 2 \\
\hline 5627.50 & $\mathrm{Fe}$ II & 3.387 & -4.14 & 8.1 & 2 \\
\hline 5991.38 & Fe II & 3.153 & -3.55 & 31.9 & 2 \\
\hline 6084.10 & Fe II & 3.200 & -3.80 & 21.5 & 2 \\
\hline 6113.33 & Fe II & 3.221 & -4.12 & 11.5 & 2 \\
\hline 6149.25 & $\mathrm{Fe}$ II & 3.889 & -2.72 & 37.6 & 2 \\
\hline 6239.95 & $\mathrm{Fe}$ II & 3.889 & -3.44 & 12.5 & 2 \\
\hline 6247.56 & $\mathrm{Fe}$ II & 3.892 & -2.32 & 55.4 & 2 \\
\hline 6369.46 & $\mathrm{Fe}$ II & 2.891 & -4.21 & 18.2 & 2 \\
\hline 6383.72 & $\mathrm{Fe}$ II & 5.553 & -2.09 & 10.2 & 2 \\
\hline 6416.93 & Fe II & 3.892 & -2.70 & 41.0 & 2 \\
\hline 6432.68 & Fe II & 2.891 & -3.58 & 43.3 & 2 \\
\hline 6456.39 & $\mathrm{Fe}$ II & 3.904 & -2.10 & 65.4 & 2 \\
\hline 6516.08 & $\mathrm{Fe}$ II & 2.891 & -3.38 & 57.0 & 2 \\
\hline
\end{tabular}

References: (1) Gratton et al. (2003); (2) Barklem et al. (2000); (3) Unsold (1955). 
P. Sestito et al.: Chemical abundances in open clusters. I., Online Material p 4

Table 9. Equivalent widths for Fe lines in stars of NGC 3960.

\begin{tabular}{|c|c|c|c|c|c|c|c|}
\hline Wavelength & Atom & $E W(\mathrm{~m} \AA)$ & & & & & \\
\hline & & c3 & c4 & $c 5$ & c6 & $\mathrm{c} 8$ & c9 \\
\hline 5494.47 & FeI & 57.6 & 60.0 & 63.9 & 59.1 & 54.9 & 60.2 \\
\hline 5521.28 & FeI & 21.7 & 0.0 & 0.0 & 0.0 & 0.0 & 16.8 \\
\hline 5522.45 & FeI & 69.3 & 66.8 & 68.2 & 68.8 & 67.1 & 69.4 \\
\hline 5524.24 & FeI & 11.4 & 17.8 & 15.8 & 0.0 & 0.0 & 13.8 \\
\hline 5539.29 & FeI & 46.1 & 39.5 & 46.4 & 43.6 & 36.3 & 41.4 \\
\hline 5547.00 & FeI & 57.4 & 56.6 & 61.0 & 58.5 & 56.1 & 59.8 \\
\hline 5552.69 & FeI & 19.5 & 17.4 & 19.5 & 16.6 & 14.5 & 13.3 \\
\hline 5560.22 & FeI & 75.3 & 72.3 & 78.2 & 75.6 & 70.2 & 71.8 \\
\hline 5568.86 & FeI & 31.0 & 29.5 & 32.8 & 31.0 & 26.6 & 28.2 \\
\hline 5577.03 & FeI & 22.5 & 24.6 & 23.5 & 26.5 & 23.7 & 27.0 \\
\hline 5586.77 & FeI & 208.6 & 201.9 & 221.6 & 209.0 & 196.5 & 208.9 \\
\hline 5587.58 & FeI & 63.1 & 63.9 & 70.6 & 70.5 & 64.2 & 59.1 \\
\hline 5595.05 & FeI & 20.3 & 12.6 & 17.0 & 14.1 & 25.9 & 6.8 \\
\hline 5608.98 & FeI & 28.8 & 25.1 & 33.0 & 27.5 & 29.7 & 29.9 \\
\hline 5609.97 & FeI & 15.8 & 16.9 & 26.0 & 0.0 & 17.0 & 16.1 \\
\hline 5611.36 & FeI & 28.2 & 26.7 & 29.0 & 24.2 & 25.6 & 24.3 \\
\hline 5618.64 & FeI & 73.5 & 73.1 & 79.0 & 76.3 & 69.6 & 75.9 \\
\hline 5619.61 & FeI & 58.2 & 56.8 & 61.8 & 60.4 & 53.8 & 60.5 \\
\hline 5635.83 & FeI & 58.8 & 58.5 & 60.9 & 58.4 & 58.8 & 64.9 \\
\hline 5636.70 & FeI & 46.7 & 45.4 & 48.9 & 44.2 & 40.2 & 43.4 \\
\hline 5650.00 & FeI & 70.0 & 64.9 & 78.6 & 80.0 & 61.0 & 66.0 \\
\hline 5651.48 & FeI & 38.2 & 35.7 & 38.3 & 35.9 & 30.8 & 33.6 \\
\hline 5652.33 & FeI & 48.8 & 49.1 & 51.8 & 47.8 & 45.2 & 45.4 \\
\hline 5661.02 & FeI & 9.5 & 12.3 & 17.0 & 0.0 & 10.2 & 9.4 \\
\hline 5661.35 & FeI & 0.0 & 47.0 & 55.6 & 49.5 & 44.8 & 47.6 \\
\hline 5677.69 & FeI & 19.0 & 19.6 & 23.7 & 22.0 & 16.1 & 17.1 \\
\hline 5678.39 & FeI & 18.1 & 12.7 & 11.7 & 19.8 & 14.3 & 10.6 \\
\hline 5680.24 & FeI & 33.0 & 30.3 & 31.1 & 33.4 & 26.9 & 31.6 \\
\hline 5701.56 & FeI & 123.8 & 121.4 & 132.2 & 128.0 & 118.4 & 123.9 \\
\hline 5717.84 & FeI & 94.2 & 90.8 & 0.0 & 92.3 & 87.0 & 89.4 \\
\hline 5731.77 & FeI & 85.1 & 83.8 & 93.5 & 85.1 & 79.1 & 88.0 \\
\hline 5738.24 & FeI & 35.2 & 29.3 & 33.8 & 29.3 & 33.0 & 30.2 \\
\hline 5741.86 & FeI & 60.8 & 56.6 & 60.6 & 57.8 & 53.0 & 55.3 \\
\hline 5742.96 & FeI & 28.6 & 38.0 & 33.3 & 26.4 & 29.2 & 33.9 \\
\hline 5752.04 & FeI & 78.9 & 77.1 & 78.5 & 77.8 & 71.6 & 75.2 \\
\hline 5754.41 & FeI & 50.4 & 42.8 & 53.7 & 57.5 & 52.9 & 32.7 \\
\hline 5759.26 & FeI & 19.0 & 14.9 & 21.8 & 22.2 & 0.0 & 20.6 \\
\hline 5760.36 & $\mathrm{FeI}$ & 50.7 & 49.6 & 55.2 & 52.6 & 47.6 & 52.7 \\
\hline 5775.09 & FeI & 82.6 & 0.0 & 0.0 & 82.9 & 0.0 & 0.0 \\
\hline 5778.46 & FeI & 0.0 & 0.0 & 0.0 & 0.0 & 0.0 & 0.0 \\
\hline 5784.67 & FeI & 0.0 & 0.0 & 0.0 & 0.0 & 0.0 & 0.0 \\
\hline 5793.92 & FeI & 0.0 & 0.0 & 0.0 & 0.0 & 0.0 & 0.0 \\
\hline 5806.73 & FeI & 0.0 & 0.0 & 0.0 & 0.0 & 0.0 & 0.0 \\
\hline 5811.91 & FeI & 0.0 & 0.0 & 0.0 & 0.0 & 0.0 & 0.0 \\
\hline 5814.81 & FeI & 0.0 & 0.0 & 0.0 & 0.0 & 0.0 & 0.0 \\
\hline 5835.11 & FeI & 39.8 & 0.0 & 51.7 & 0.0 & 37.5 & 0.0 \\
\hline 5837.70 & FeI & 23.5 & 0.0 & 28.2 & 23.0 & 21.9 & 18.7 \\
\hline 5849.69 & FeI & 27.7 & 0.0 & 28.7 & 26.6 & 27.4 & 0.0 \\
\hline 5852.23 & FeI & 73.1 & 69.9 & 74.6 & 67.3 & 65.6 & 65.0 \\
\hline 5853.15 & FeI & 34.8 & 39.4 & 39.8 & 35.7 & 30.9 & 32.1 \\
\hline 5855.09 & FeI & 41.2 & 42.2 & 42.7 & 39.1 & 39.6 & 40.6 \\
\hline 5856.10 & FeI & 60.4 & 62.1 & 62.0 & 61.6 & 54.5 & 59.4 \\
\hline 5858.78 & FeI & 31.0 & 30.4 & 35.0 & 30.5 & 27.3 & 30.1 \\
\hline 5859.60 & FeI & 95.4 & 92.6 & 98.1 & 95.6 & 89.4 & 96.9 \\
\hline 5861.11 & FeI & 17.7 & 17.4 & 24.3 & 21.0 & 18.5 & 21.3 \\
\hline 5862.37 & FeI & 102.2 & 103.4 & 109.3 & 105.8 & 102.2 & 108.6 \\
\hline 5879.49 & FeI & 32.2 & 27.4 & 27.0 & 25.5 & 25.4 & 27.5 \\
\hline 5880.02 & FeI & 30.8 & 32.4 & 32.2 & 29.5 & 27.3 & 31.6 \\
\hline 5881.28 & FeI & 37.3 & 35.6 & 40.6 & 35.4 & 33.2 & 36.0 \\
\hline 5902.48 & FeI & 35.3 & 25.4 & 33.4 & 37.5 & 23.1 & 26.4 \\
\hline 5905.68 & FeI & 78.8 & 82.7 & 82.8 & 82.9 & 73.8 & 80.4 \\
\hline 5927.80 & FeI & 62.3 & 59.8 & 69.4 & 63.8 & 58.3 & 64.4 \\
\hline
\end{tabular}


P. Sestito et al.: Chemical abundances in open clusters. I., Online Material p 5

Table 9. continued.

\begin{tabular}{|c|c|c|c|c|c|c|c|}
\hline $\begin{array}{l}\text { Wavelength } \\
\text { (§) }\end{array}$ & Atom & $\begin{array}{l}E W(\mathrm{~m} \AA) \\
\mathrm{c} 3\end{array}$ & c4 & c5 & c6 & $\mathrm{c} 8$ & c9 \\
\hline 5929.68 & FeI & 64.6 & 66.4 & 64.3 & 63.2 & 59.3 & 65.0 \\
\hline 5930.19 & $\mathrm{FeI}$ & 0.0 & 0.0 & 0.0 & 0.0 & 0.0 & 0.0 \\
\hline 5933.81 & FeI & 16.6 & 16.7 & 20.4 & 18.9 & 13.7 & 19.0 \\
\hline 5934.66 & $\mathrm{FeI}$ & 103.9 & 102.2 & 105.9 & 104.9 & 97.4 & 107.1 \\
\hline 5947.53 & FeI & 25.2 & 30.0 & 34.2 & 28.0 & 25.6 & 26.1 \\
\hline 5956.71 & FeI & 105.6 & 102.4 & 115.0 & 109.2 & 100.6 & 106.4 \\
\hline 5976.79 & FeI & 94.6 & 93.6 & 101.8 & 95.1 & 90.6 & 93.9 \\
\hline 5984.83 & $\mathrm{FeI}$ & 105.7 & 102.4 & 106.9 & 104.0 & 99.6 & 103.5 \\
\hline 6003.02 & $\mathrm{FeI}$ & 106.1 & 112.5 & 111.3 & 112.1 & 102.4 & 108.2 \\
\hline 6007.97 & $\mathrm{FeI}$ & 84.4 & 81.3 & 85.5 & 82.8 & 80.6 & 81.3 \\
\hline 6008.57 & FeI & 111.1 & 111.9 & 114.6 & 113.3 & 107.5 & 111.2 \\
\hline 6015.24 & FeI & 23.0 & 19.5 & 27.1 & 20.8 & 20.0 & 21.1 \\
\hline 6019.37 & FeI & 27.4 & 17.6 & 26.2 & 16.7 & 18.1 & 17.9 \\
\hline 6027.06 & FeI & 93.4 & 93.7 & 99.9 & 99.6 & 91.2 & 99.4 \\
\hline 6056.01 & FeI & 89.1 & 88.7 & 96.6 & 94.5 & 84.5 & 90.0 \\
\hline 6065.49 & FeI & 164.8 & 162.2 & 170.8 & 165.7 & 158.4 & 166.2 \\
\hline 6078.50 & $\mathrm{FeI}$ & 101.7 & 99.9 & 96.0 & 99.7 & 91.6 & 102.8 \\
\hline 6079.02 & $\mathrm{FeI}$ & 69.0 & 70.9 & 76.4 & 74.3 & 66.7 & 71.3 \\
\hline 6082.72 & FeI & 81.3 & 79.6 & 84.6 & 82.4 & 74.1 & 80.8 \\
\hline 6089.57 & FeI & 64.2 & 62.6 & 68.2 & 64.4 & 61.7 & 64.7 \\
\hline 6093.65 & FeI & 50.2 & 49.1 & 54.2 & 50.7 & 45.9 & 50.9 \\
\hline 6094.38 & FeI & 39.3 & 37.0 & 39.1 & 36.9 & 35.4 & 35.8 \\
\hline 6096.67 & $\mathrm{FeI}$ & 62.8 & 66.6 & 66.1 & 65.5 & 59.5 & 61.3 \\
\hline 6098.25 & $\mathrm{FeI}$ & 35.2 & 35.4 & 36.8 & 37.1 & 34.1 & 34.6 \\
\hline 6120.26 & FeI & 31.7 & 28.2 & 35.1 & 34.4 & 24.8 & 32.1 \\
\hline 6137.00 & FeI & 119.2 & 122.0 & 127.8 & 121.3 & 137.7 & 116.8 \\
\hline 6151.62 & FeI & 92.4 & 90.2 & 97.6 & 91.9 & 89.4 & 93.4 \\
\hline 6157.73 & FeI & 99.5 & 98.8 & 104.6 & 99.6 & 94.1 & 100.2 \\
\hline 6165.36 & FeI & 74.9 & 71.6 & 77.3 & 73.8 & 68.5 & 73.8 \\
\hline 6173.34 & FeI & 112.1 & 109.7 & 121.2 & 116.1 & 106.3 & 111.8 \\
\hline 6187.40 & $\mathrm{FeI}$ & 27.8 & 21.5 & 22.2 & 18.4 & 18.8 & 16.6 \\
\hline 6187.99 & $\mathrm{FeI}$ & 78.9 & 79.3 & 80.4 & 79.6 & 72.3 & 80.5 \\
\hline 6199.51 & FeI & 21.9 & 19.4 & 26.2 & 27.3 & 19.6 & 17.9 \\
\hline 6200.32 & FeI & 112.9 & 112.7 & 116.4 & 115.2 & 109.3 & 112.5 \\
\hline 6213.44 & FeI & 128.4 & 128.5 & 136.2 & 132.2 & 125.9 & 127.9 \\
\hline 6219.29 & FeI & 137.7 & 134.4 & 148.9 & 139.6 & 131.4 & 139.3 \\
\hline 6220.79 & FeI & 45.7 & 46.1 & 49.4 & 45.2 & 41.7 & 44.5 \\
\hline 6226.74 & FeI & 52.7 & 55.1 & 60.1 & 56.5 & 54.5 & 53.9 \\
\hline 6232.65 & FeI & 114.0 & 113.7 & 119.1 & 118.1 & 111.3 & 116.7 \\
\hline 6240.65 & FeI & 90.9 & 92.2 & 98.7 & 92.0 & 87.1 & 91.4 \\
\hline 6246.33 & FeI & 0.0 & 0.0 & 0.0 & 0.0 & 0.0 & 0.0 \\
\hline 6252.56 & FeI & 166.9 & 161.8 & 172.8 & 166.8 & 156.9 & 162.3 \\
\hline 6265.14 & FeI & 135.6 & 135.5 & 143.8 & 139.6 & 126.4 & 135.5 \\
\hline 6270.23 & FeI & 99.1 & 98.6 & 101.2 & 99.5 & 96.2 & 99.9 \\
\hline 6280.62 & $\mathrm{FeI}$ & 162.5 & 165.9 & 139.1 & 166.3 & 146.0 & 148.5 \\
\hline 6290.55 & FeI & 0.0 & 32.5 & 48.3 & 0.0 & 23.0 & 40.6 \\
\hline 6297.80 & FeI & 123.1 & 117.5 & 131.3 & 128.3 & 118.1 & 119.1 \\
\hline 6301.51 & FeI & 0.0 & 0.0 & 0.0 & 0.0 & 0.0 & 0.0 \\
\hline 6303.47 & FeI & 34.0 & 21.9 & 11.7 & 28.3 & 41.9 & 13.5 \\
\hline 6311.50 & FeI & 61.1 & 67.3 & 70.2 & 64.5 & 72.4 & 63.4 \\
\hline 6315.81 & FeI & 67.7 & 69.8 & 72.5 & 69.0 & 72.5 & 70.9 \\
\hline 6322.69 & FeI & 115.5 & 115.8 & 124.3 & 119.3 & 109.7 & 118.3 \\
\hline 6330.85 & FeI & 52.4 & 54.7 & 58.9 & 52.0 & 52.9 & 56.1 \\
\hline 6335.34 & FeI & 143.8 & 146.8 & 152.8 & 147.8 & 141.5 & 148.0 \\
\hline 6380.75 & FeI & 85.6 & 83.7 & 91.0 & 87.3 & 83.6 & 85.7 \\
\hline 6392.54 & FeI & 54.6 & 50.3 & 56.6 & 52.4 & 52.1 & 51.7 \\
\hline 6393.61 & FeI & 180.4 & 178.8 & 189.8 & 181.9 & 170.6 & 176.4 \\
\hline 6400.32 & FeI & 120.4 & 141.3 & 144.6 & 136.1 & 131.8 & 122.6 \\
\hline 6411.11 & FeI & 18.3 & 0.0 & 25.2 & 0.0 & 21.4 & 0.0 \\
\hline 6411.66 & FeI & 0.0 & 0.0 & 0.0 & 0.0 & 0.0 & 0.0 \\
\hline 6421.36 & FeI & 173.5 & 169.2 & 184.8 & 177.7 & 167.7 & 171.0 \\
\hline 6436.41 & FeI & 27.2 & 23.7 & 25.9 & 28.3 & 27.5 & 27.2 \\
\hline 6481.88 & FeI & 111.4 & 109.6 & 117.6 & 111.6 & 105.2 & 110.8 \\
\hline
\end{tabular}


P. Sestito et al.: Chemical abundances in open clusters. I., Online Material p 6

Table 9. continued.

\begin{tabular}{|c|c|c|c|c|c|c|c|}
\hline $\begin{array}{l}\text { Wavelength } \\
(\AA)\end{array}$ & Atom & $\begin{array}{l}E W(\mathrm{~m} \AA) \\
\mathrm{c} 3\end{array}$ & c4 & $\mathrm{c} 5$ & c6 & $\mathrm{c} 8$ & c9 \\
\hline 6498.94 & FeI & 99.7 & 96.8 & 106.2 & 101.9 & 97.1 & 98.0 \\
\hline 6518.37 & FeI & 91.0 & 93.3 & 103.7 & 96.2 & 88.0 & 94.9 \\
\hline 6533.94 & FeI & 62.4 & 66.6 & 62.9 & 62.7 & 58.7 & 52.9 \\
\hline 6574.25 & $\mathrm{FeI}$ & 82.6 & 82.1 & 90.2 & 84.0 & 74.0 & 81.4 \\
\hline 6581.22 & FeI & 74.6 & 71.3 & 82.3 & 75.9 & 68.2 & 72.7 \\
\hline 6591.31 & FeI & 23.2 & 23.9 & 25.4 & 23.0 & 23.9 & 24.6 \\
\hline 6593.88 & FeI & 128.5 & 134.2 & 139.5 & 134.0 & 130.2 & 134.5 \\
\hline 6608.04 & FeI & 55.3 & 50.4 & 64.4 & 53.5 & 53.4 & 56.4 \\
\hline 6609.12 & FeI & 113.6 & 108.3 & 120.8 & 112.8 & 106.7 & 107.9 \\
\hline 6625.04 & FeI & 68.8 & 69.1 & 80.4 & 75.9 & 63.5 & 73.2 \\
\hline 6627.56 & FeI & 50.2 & 52.7 & 55.6 & 50.5 & 47.4 & 49.5 \\
\hline 6633.76 & FeI & 91.5 & 91.8 & 95.3 & 92.7 & 91.2 & 93.7 \\
\hline 6667.43 & FeI & 25.6 & 26.8 & 31.6 & 27.8 & 28.0 & 30.4 \\
\hline 6667.72 & FeI & 28.1 & 26.5 & 27.4 & 26.7 & 23.4 & 23.5 \\
\hline 6699.14 & FeI & 17.8 & 19.8 & 17.9 & 19.4 & 16.3 & 14.9 \\
\hline 6703.58 & FeI & 74.3 & 75.3 & 80.7 & 76.6 & 70.5 & 73.2 \\
\hline 6704.48 & FeI & 15.8 & 17.1 & 16.4 & 13.7 & 15.9 & 14.4 \\
\hline 6713.74 & FeI & 41.6 & 41.0 & 39.0 & 40.3 & 35.9 & 36.1 \\
\hline 6725.36 & FeI & 40.2 & 38.0 & 40.1 & 39.6 & 34.7 & 36.4 \\
\hline 6726.67 & FeI & 71.7 & 67.8 & 72.8 & 69.0 & 66.9 & 67.0 \\
\hline 6733.15 & FeI & 41.8 & 48.3 & 51.9 & 49.7 & 46.6 & 46.7 \\
\hline 6739.52 & FeI & 46.5 & 42.6 & 50.0 & 43.2 & 39.7 & 45.1 \\
\hline 6745.97 & FeI & 19.6 & 18.7 & 21.6 & 17.0 & 17.5 & 18.2 \\
\hline 6750.16 & FeI & 128.3 & 121.7 & 129.3 & 0.0 & 115.5 & 126.4 \\
\hline 6753.47 & FeI & 18.1 & 15.9 & 17.9 & 17.2 & 17.8 & 0.0 \\
\hline 6756.55 & FeI & 9.9 & 8.6 & 12.5 & 0.0 & 10.6 & 0.0 \\
\hline 6786.86 & FeI & 50.2 & 48.1 & 55.3 & 50.5 & 47.7 & 49.5 \\
\hline 6793.26 & FeI & 31.9 & 26.0 & 35.3 & 32.2 & 33.3 & 30.0 \\
\hline 6796.12 & FeI & 36.9 & 29.9 & 41.6 & 37.1 & 37.3 & 39.4 \\
\hline 6804.30 & FeI & 38.5 & 34.0 & 34.6 & 38.4 & 31.4 & 35.9 \\
\hline 6806.86 & FeI & 78.5 & 65.9 & 79.0 & 78.4 & 73.4 & 75.8 \\
\hline 6810.27 & FeI & 77.7 & 0.0 & 78.0 & 73.6 & 72.1 & 74.8 \\
\hline 5525.14 & FeII & 42.5 & 39.5 & 41.5 & 34.3 & 40.3 & 39.2 \\
\hline 5534.85 & FeII & 94.7 & 97.0 & 105.0 & 91.3 & 92.2 & 97.6 \\
\hline 5627.50 & FeII & 23.1 & 0.0 & 34.0 & 21.9 & 0.0 & 0.0 \\
\hline 5991.38 & FeII & 58.9 & 58.5 & 62.4 & 61.7 & 56.8 & 59.5 \\
\hline 6084.10 & FeII & 46.0 & 40.9 & 43.7 & 42.6 & 37.6 & 47.0 \\
\hline 6113.33 & FeII & 34.1 & 36.5 & 33.3 & 37.2 & 30.1 & 34.2 \\
\hline 6149.25 & FeII & 52.1 & 50.7 & 58.1 & 54.9 & 49.3 & 55.7 \\
\hline 6239.95 & FeII & 41.2 & 37.9 & 27.2 & 51.9 & 37.4 & 43.7 \\
\hline 6247.56 & FeII & 73.6 & 74.0 & 74.5 & 73.5 & 69.8 & 72.6 \\
\hline 6369.46 & FeII & 40.5 & 42.4 & 42.9 & 40.4 & 39.7 & 40.9 \\
\hline 6383.72 & FeII & 17.5 & 17.9 & 18.2 & 15.8 & 23.0 & 13.3 \\
\hline 6416.93 & FeII & 58.7 & 60.3 & 57.5 & 59.4 & 57.2 & 60.9 \\
\hline 6432.68 & FeII & 65.9 & 64.8 & 66.5 & 66.4 & 68.0 & 65.7 \\
\hline 6456.39 & FeII & 83.5 & 87.3 & 84.7 & 86.3 & 83.1 & 86.9 \\
\hline 6516.08 & FeII & 81.2 & 81.0 & 85.8 & 86.8 & 79.0 & 89.2 \\
\hline
\end{tabular}


P. Sestito et al.: Chemical abundances in open clusters. I., Online Material $p 7$

Table 10. Equivalent widths for Fe lines in stars of $\mathrm{Be} 32$.

\begin{tabular}{|c|c|c|c|c|c|c|c|c|c|c|}
\hline \multirow{2}{*}{$\begin{array}{l}\text { Wavelength } \\
\text { (̊̊) }\end{array}$} & \multirow[t]{2}{*}{ Atom } & \multicolumn{9}{|l|}{$E W(\mathrm{~m} \AA)$} \\
\hline & & 17 & 18 & 19 & 25 & 27 & 45 & 938 & 940 & 941 \\
\hline 5494.47 & FeI & 40.6 & 45.3 & 45.4 & 50.7 & 56.7 & 38.6 & 40.7 & 41.9 & 42.3 \\
\hline 5521.28 & FeI & 0.0 & 11.5 & 0.0 & 0.0 & 0.0 & 0.0 & 0.0 & 0.0 & 0.0 \\
\hline 5522.45 & FeI & 59.5 & 61.4 & 61.7 & 63.3 & 59.1 & 58.0 & 57.9 & 60.2 & 60.9 \\
\hline 5524.24 & FeI & 0.0 & 0.0 & 10.3 & 0.0 & 13.9 & 0.0 & 0.0 & 0.0 & 0.0 \\
\hline 5539.29 & FeI & 39.2 & 37.9 & 34.6 & 43.6 & 41.8 & 0.0 & 34.9 & 32.8 & 33.8 \\
\hline 5547.00 & FeI & 46.8 & 50.9 & 46.1 & 67.4 & 80.8 & 27.7 & 46.0 & 53.3 & 57.0 \\
\hline 5552.69 & FeI & 0.0 & 0.0 & 11.6 & 14.7 & 11.1 & 0.0 & 9.1 & 13.7 & 11.0 \\
\hline 5560.22 & FeI & 61.8 & 62.4 & 64.3 & 69.1 & 65.6 & 57.8 & 63.2 & 66.2 & 67.8 \\
\hline 5568.86 & FeI & 20.6 & 19.9 & 30.4 & 24.3 & 23.9 & 18.1 & 21.2 & 18.9 & 26.5 \\
\hline 5577.03 & FeI & 18.6 & 21.4 & 0.0 & 15.5 & 16.7 & 0.0 & 0.0 & 0.0 & 19.4 \\
\hline 5586.77 & FeI & 189.2 & 187.5 & 192.4 & 211.0 & 207.2 & 205.9 & 177.4 & 196.7 & 188.7 \\
\hline 5587.58 & FeI & 54.5 & 52.2 & 64.0 & 68.0 & 67.9 & 54.8 & 52.8 & 54.6 & 58.2 \\
\hline 5595.05 & FeI & 14.0 & 10.0 & 0.0 & 25.8 & 22.3 & 0.0 & 12.6 & 0.0 & 11.3 \\
\hline 5608.98 & FeI & 19.8 & 20.1 & 19.5 & 0.0 & 30.2 & 15.6 & 0.0 & 20.1 & 27.7 \\
\hline 5609.97 & FeI & 0.0 & 17.1 & 0.0 & 20.5 & 0.0 & 15.2 & 10.1 & 0.0 & 15.2 \\
\hline 5611.36 & FeI & 24.4 & 18.7 & 24.7 & 23.0 & 23.0 & 11.1 & 23.6 & 19.5 & 23.4 \\
\hline 5618.64 & FeI & 69.7 & 64.4 & 63.1 & 76.1 & 73.4 & 62.0 & 64.0 & 63.4 & 67.8 \\
\hline 5619.61 & FeI & 47.0 & 51.4 & 46.6 & 49.3 & 53.7 & 39.8 & 50.9 & 48.8 & 53.5 \\
\hline 5635.83 & FeI & 47.8 & 58.6 & 50.6 & 46.5 & 53.7 & 48.6 & 49.8 & 48.0 & 55.4 \\
\hline 5636.70 & FeI & 33.6 & 40.6 & 38.2 & 47.1 & 46.3 & 31.8 & 34.9 & 41.3 & 41.8 \\
\hline 5650.00 & FeI & 44.0 & 68.0 & 52.0 & 68.5 & 48.0 & 40.0 & 43.0 & 41.0 & 52.0 \\
\hline 5651.48 & FeI & 31.2 & 28.4 & 30.3 & 28.3 & 29.6 & 27.0 & 23.4 & 28.9 & 29.9 \\
\hline 5652.33 & FeI & 38.3 & 38.9 & 45.3 & 45.8 & 44.0 & 29.7 & 41.5 & 43.3 & 42.6 \\
\hline 5661.02 & FeI & 5.5 & 0.0 & 11.8 & 0.0 & 0.0 & 0.0 & 0.0 & 0.0 & 0.0 \\
\hline 5661.35 & FeI & 39.5 & 41.8 & 34.5 & 46.5 & 48.1 & 28.3 & 34.0 & 33.8 & 41.2 \\
\hline 5677.69 & FeI & 16.6 & 15.1 & 16.6 & 0.0 & 19.7 & 0.0 & 0.0 & 0.0 & 15.1 \\
\hline 5678.39 & FeI & 10.4 & 17.4 & 10.6 & 0.0 & 0.0 & 0.0 & 0.0 & 0.0 & 0.0 \\
\hline 5680.24 & FeI & 25.6 & 26.2 & 21.7 & 20.9 & 25.6 & 0.0 & 24.3 & 26.2 & 22.8 \\
\hline 5701.56 & FeI & 116.7 & 118.8 & 118.4 & 123.9 & 123.2 & 97.2 & 118.0 & 119.5 & 116.3 \\
\hline 5717.84 & FeI & 80.9 & 79.8 & 80.5 & 82.3 & 82.4 & 65.7 & 74.4 & 84.1 & 85.1 \\
\hline 5731.77 & FeI & 72.3 & 76.4 & 75.9 & 89.2 & 83.5 & 70.3 & 79.8 & 85.8 & 83.2 \\
\hline 5738.24 & FeI & 27.8 & 36.4 & 21.3 & 0.0 & 33.7 & 0.0 & 0.0 & 31.1 & 37.7 \\
\hline 5741.86 & FeI & 46.4 & 61.9 & 56.0 & 86.1 & 65.0 & 0.0 & 63.6 & 57.6 & 52.6 \\
\hline 5742.96 & FeI & 0.0 & 0.0 & 0.0 & 0.0 & 36.1 & 23.8 & 0.0 & 0.0 & 31.4 \\
\hline 5752.04 & FeI & 0.0 & 0.0 & 0.0 & 0.0 & 0.0 & 0.0 & 0.0 & 0.0 & 0.0 \\
\hline 5754.41 & FeI & 0.0 & 0.0 & 0.0 & 0.0 & 0.0 & 0.0 & 0.0 & 0.0 & 0.0 \\
\hline 5759.26 & FeI & 0.0 & 0.0 & 0.0 & 0.0 & 0.0 & 0.0 & 0.0 & 0.0 & 0.0 \\
\hline 5760.36 & FeI & 0.0 & 0.0 & 0.0 & 0.0 & 0.0 & 0.0 & 0.0 & 0.0 & 0.0 \\
\hline 5775.09 & FeI & 0.0 & 0.0 & 0.0 & 0.0 & 0.0 & 0.0 & 0.0 & 0.0 & 0.0 \\
\hline 5778.46 & FeI & 0.0 & 0.0 & 0.0 & 0.0 & 0.0 & 0.0 & 0.0 & 0.0 & 0.0 \\
\hline 5784.67 & FeI & 0.0 & 0.0 & 0.0 & 0.0 & 0.0 & 0.0 & 0.0 & 0.0 & 0.0 \\
\hline 5793.92 & FeI & 0.0 & 0.0 & 0.0 & 0.0 & 0.0 & 0.0 & 0.0 & 0.0 & 0.0 \\
\hline 5806.73 & FeI & 0.0 & 0.0 & 0.0 & 0.0 & 0.0 & 0.0 & 0.0 & 0.0 & 0.0 \\
\hline 5811.91 & FeI & 0.0 & 0.0 & 0.0 & 0.0 & 0.0 & 0.0 & 0.0 & 0.0 & 0.0 \\
\hline 5814.81 & FeI & 0.0 & 0.0 & 0.0 & 0.0 & 0.0 & 0.0 & 0.0 & 0.0 & 0.0 \\
\hline 5835.11 & FeI & 25.9 & 0.0 & 43.1 & 51.2 & 0.0 & 0.0 & 21.5 & 0.0 & 35.7 \\
\hline 5837.70 & FeI & 20.9 & 0.0 & 0.0 & 0.0 & 19.3 & 0.0 & 0.0 & 24.3 & 29.4 \\
\hline 5849.69 & FeI & 16.4 & 0.0 & 12.1 & 28.0 & 16.7 & 0.0 & 18.9 & 20.2 & 20.3 \\
\hline 5852.23 & FeI & 54.9 & 54.4 & 55.1 & 71.9 & 62.2 & 47.0 & 48.5 & 64.9 & 61.5 \\
\hline 5853.15 & FeI & 30.5 & 38.1 & 28.8 & 43.5 & 38.3 & 29.9 & 29.2 & 37.4 & 36.4 \\
\hline 5855.09 & FeI & 25.5 & 31.1 & 33.6 & 37.7 & 37.9 & 34.9 & 32.4 & 32.3 & 31.5 \\
\hline 5856.10 & FeI & 48.4 & 53.9 & 42.1 & 62.3 & 47.8 & 38.6 & 48.0 & 46.7 & 48.3 \\
\hline 5858.78 & FeI & 19.8 & 21.5 & 24.1 & 26.2 & 21.8 & 25.3 & 24.6 & 24.5 & 0.0 \\
\hline 5859.60 & FeI & 87.8 & 84.7 & 89.8 & 93.0 & 88.1 & 84.5 & 88.7 & 88.2 & 75.3 \\
\hline 5861.11 & FeI & 11.2 & 14.3 & 20.4 & 26.0 & 19.1 & 0.0 & 0.0 & 0.0 & 12.0 \\
\hline 5862.37 & FeI & 92.9 & 95.3 & 91.9 & 99.3 & 75.0 & 82.7 & 90.9 & 91.0 & 92.4 \\
\hline 5879.49 & FeI & 0.0 & 21.4 & 19.4 & 0.0 & 31.6 & 15.9 & 0.0 & 0.0 & 0.0 \\
\hline 5880.02 & FeI & 20.2 & 18.6 & 24.3 & 27.5 & 28.9 & 0.0 & 0.0 & 0.0 & 24.5 \\
\hline 5881.28 & FeI & 28.6 & 25.6 & 25.9 & 28.4 & 37.1 & 0.0 & 0.0 & 24.9 & 27.3 \\
\hline 5902.48 & FeI & 21.7 & 26.7 & 17.9 & 25.8 & 29.7 & 18.9 & 18.5 & 21.4 & 27.7 \\
\hline 5905.68 & FeI & 71.5 & 76.1 & 69.8 & 84.9 & 73.6 & 66.0 & 71.5 & 78.3 & 79.2 \\
\hline 5927.80 & FeI & 52.1 & 52.1 & 54.5 & 54.4 & 59.3 & 43.1 & 48.1 & 45.3 & 51.6 \\
\hline
\end{tabular}


Table 10. continued.

\begin{tabular}{|c|c|c|c|c|c|c|c|c|c|c|}
\hline Wavelength & Atom & $E W(\mathrm{~m} \AA)$ & & & & & & & & \\
\hline & & 17 & 18 & 19 & 25 & 27 & 45 & 938 & 940 & 941 \\
\hline 5929.68 & FeI & 52.0 & 58.1 & 44.9 & 63.2 & 62.9 & 51.3 & 61.2 & 58.1 & 53.5 \\
\hline 5930.19 & FeI & 0.0 & 0.0 & 0.0 & 0.0 & 0.0 & 0.0 & 0.0 & 0.0 & 0.0 \\
\hline 5933.81 & FeI & 0.0 & 23.0 & 0.0 & 0.0 & 17.7 & 0.0 & 0.0 & 0.0 & 0.0 \\
\hline 5934.66 & FeI & 97.6 & 94.3 & 94.2 & 99.7 & 100.9 & 91.4 & 86.5 & 99.4 & 96.9 \\
\hline 5947.53 & FeI & 0.0 & 27.3 & 25.3 & 23.4 & 41.3 & 35.8 & 28.3 & 27.1 & 26.5 \\
\hline 5956.71 & FeI & 107.3 & 108.5 & 110.1 & 109.9 & 111.9 & 96.8 & 100.6 & 101.7 & 110.7 \\
\hline 5976.79 & FeI & 85.1 & 92.1 & 85.2 & 97.8 & 88.6 & 78.2 & 84.9 & 91.9 & 91.8 \\
\hline 5984.83 & FeI & 100.1 & 95.3 & 93.1 & 99.2 & 97.5 & 82.2 & 94.8 & 104.0 & 98.9 \\
\hline 6003.02 & FeI & 97.1 & 98.5 & 96.6 & 99.3 & 97.3 & 84.4 & 105.6 & 103.2 & 101.7 \\
\hline 6007.97 & FeI & 71.9 & 74.1 & 77.3 & 75.0 & 72.6 & 74.8 & 73.6 & 72.4 & 76.7 \\
\hline 6008.57 & FeI & 102.7 & 101.3 & 102.6 & 104.8 & 95.5 & 95.5 & 102.6 & 107.4 & 104.4 \\
\hline 6015.24 & FeI & 12.8 & 23.5 & 17.4 & 27.3 & 25.9 & 0.0 & 21.4 & 21.7 & 17.7 \\
\hline 6019.37 & FeI & 15.6 & 16.6 & 21.8 & 24.2 & 17.9 & 0.0 & 0.0 & 0.0 & 0.0 \\
\hline 6027.06 & FeI & 87.4 & 87.8 & 90.7 & 88.5 & 83.3 & 68.2 & 84.3 & 85.2 & 86.7 \\
\hline 6056.01 & FeI & 80.9 & 82.9 & 89.1 & 81.7 & 87.1 & 74.1 & 77.8 & 81.9 & 83.1 \\
\hline 6065.49 & FeI & 151.9 & 158.8 & 159.1 & 156.5 & 165.4 & 142.7 & 155.8 & 160.0 & 159.5 \\
\hline 6078.50 & FeI & 85.2 & 81.8 & 82.9 & 84.2 & 85.1 & 90.6 & 86.4 & 85.7 & 91.5 \\
\hline 6079.02 & FeI & 59.8 & 60.4 & 54.2 & 53.8 & 66.0 & 64.7 & 63.1 & 58.9 & 65.5 \\
\hline 6082.72 & FeI & 72.3 & 72.4 & 77.6 & 72.1 & 78.5 & 62.8 & 80.0 & 79.0 & 78.3 \\
\hline 6089.57 & FeI & 57.8 & 49.4 & 51.3 & 59.8 & 66.5 & 43.7 & 56.4 & 52.5 & 58.5 \\
\hline 6093.65 & FeI & 37.9 & 42.2 & 42.5 & 38.3 & 42.0 & 35.2 & 33.3 & 45.0 & 38.3 \\
\hline 6094.38 & FeI & 23.0 & 31.4 & 27.3 & 0.0 & 30.6 & 23.3 & 33.8 & 30.5 & 28.6 \\
\hline 6096.67 & FeI & 44.5 & 57.5 & 54.5 & 62.5 & 63.5 & 48.5 & 51.6 & 60.8 & 57.7 \\
\hline 6098.25 & FeI & 26.5 & 26.2 & 22.5 & 42.1 & 35.1 & 18.0 & 23.3 & 29.6 & 32.2 \\
\hline 6120.26 & FeI & 28.7 & 31.6 & 29.2 & 42.2 & 33.4 & 22.4 & 25.4 & 30.3 & 27.3 \\
\hline 6137.00 & FeI & 114.3 & 109.1 & 112.9 & 113.6 & 114.0 & 97.4 & 99.4 & 114.3 & 116.8 \\
\hline 6151.62 & FeI & 87.3 & 88.4 & 86.2 & 88.1 & 88.5 & 68.6 & 81.5 & 81.6 & 86.6 \\
\hline 6157.73 & FeI & 94.3 & 91.7 & 83.6 & 88.8 & 88.9 & 75.4 & 84.7 & 90.9 & 90.7 \\
\hline 6165.36 & FeI & 60.9 & 64.2 & 70.5 & 66.0 & 67.4 & 63.6 & 60.5 & 67.7 & 64.1 \\
\hline 6173.34 & FeI & 108.9 & 112.1 & 110.0 & 109.8 & 107.0 & 96.6 & 104.6 & 113.6 & 115.1 \\
\hline 6187.40 & FeI & 17.6 & 14.1 & 0.0 & 23.1 & 18.2 & 0.0 & 0.0 & 0.0 & 13.5 \\
\hline 6187.99 & FeI & 68.1 & 66.9 & 69.3 & 71.9 & 71.6 & 62.2 & 69.4 & 69.7 & 68.0 \\
\hline 6199.51 & FeI & 0.0 & 23.2 & 26.4 & 28.7 & 28.2 & 13.1 & 24.8 & 0.0 & 20.6 \\
\hline 6200.32 & FeI & 107.6 & 107.2 & 102.3 & 105.7 & 105.8 & 106.5 & 95.0 & 105.6 & 109.5 \\
\hline 6213.44 & FeI & 130.5 & 128.8 & 127.2 & 117.8 & 133.6 & 105.2 & 115.8 & 126.3 & 129.2 \\
\hline 6219.29 & FeI & 131.1 & 128.6 & 132.5 & 131.5 & 132.5 & 110.6 & 124.7 & 128.7 & 135.1 \\
\hline 6220.79 & FeI & 37.2 & 32.4 & 33.3 & 44.2 & 42.7 & 26.5 & 34.6 & 34.6 & 41.3 \\
\hline 6226.74 & FeI & 46.3 & 46.8 & 47.0 & 51.8 & 56.4 & 47.2 & 42.2 & 44.4 & 49.5 \\
\hline 6232.65 & FeI & 106.7 & 104.5 & 105.3 & 117.9 & 103.0 & 86.3 & 99.7 & 110.0 & 110.3 \\
\hline 6240.65 & FeI & 89.2 & 85.3 & 84.8 & 92.3 & 88.0 & 72.8 & 90.6 & 92.8 & 91.1 \\
\hline 6246.33 & FeI & 0.0 & 0.0 & 0.0 & 0.0 & 0.0 & 0.0 & 0.0 & 0.0 & 0.0 \\
\hline 6252.56 & FeI & 160.4 & 161.8 & 158.2 & 167.2 & 166.7 & 142.7 & 149.4 & 161.9 & 162.3 \\
\hline 6265.14 & FeI & 129.5 & 131.3 & 128.0 & 135.6 & 135.4 & 123.5 & 128.6 & 133.3 & 135.1 \\
\hline 6270.23 & FeI & 85.8 & 84.2 & 88.4 & 102.0 & 77.5 & 78.2 & 81.2 & 91.3 & 90.6 \\
\hline 6280.62 & FeI & 133.6 & 129.2 & 142.7 & 141.8 & 136.2 & 111.1 & 123.6 & 132.8 & 136.4 \\
\hline 6290.55 & FeI & 43.4 & 44.6 & 0.0 & 45.5 & 46.8 & 41.4 & 40.8 & 42.5 & 47.9 \\
\hline 6297.80 & FeI & 115.5 & 111.3 & 104.3 & 126.0 & 114.8 & 87.3 & 108.5 & 116.4 & 119.4 \\
\hline 6301.51 & FeI & 0.0 & 0.0 & 0.0 & 0.0 & 0.0 & 0.0 & 0.0 & 0.0 & 0.0 \\
\hline 6303.47 & FeI & 31.2 & 30.5 & 33.4 & 34.4 & 30.1 & 26.4 & 0.0 & 32.4 & 29.5 \\
\hline 6311.50 & FeI & 61.0 & 43.7 & 51.4 & 61.5 & 63.7 & 55.8 & 59.0 & 53.9 & 57.7 \\
\hline 6315.81 & FeI & 62.4 & 60.8 & 59.0 & 65.2 & 62.2 & 48.7 & 60.9 & 58.7 & 58.7 \\
\hline 6322.69 & FeI & 111.4 & 111.1 & 104.4 & 120.3 & 117.7 & 106.9 & 112.8 & 113.4 & 113.6 \\
\hline 6330.85 & FeI & 46.2 & 45.6 & 45.5 & 54.9 & 48.5 & 47.3 & 40.9 & 43.6 & 46.6 \\
\hline 6335.34 & FeI & 143.3 & 143.3 & 140.7 & 142.6 & 140.8 & 133.2 & 141.8 & 142.8 & 143.7 \\
\hline 6380.75 & FeI & 76.1 & 76.7 & 78.4 & 87.9 & 75.0 & 76.8 & 71.1 & 74.5 & 80.7 \\
\hline 6392.54 & FeI & 46.4 & 49.6 & 45.2 & 65.4 & 58.4 & 41.9 & 50.1 & 50.5 & 47.1 \\
\hline 6393.61 & FeI & 167.7 & 172.1 & 165.0 & 172.4 & 174.8 & 137.2 & 173.7 & 173.4 & 166.1 \\
\hline 6400.32 & FeI & 134.2 & 130.7 & 141.7 & 127.5 & 134.2 & 102.3 & 111.8 & 113.7 & 135.7 \\
\hline 6411.11 & FeI & 0.0 & 0.0 & 17.0 & 0.0 & 13.3 & 0.0 & 20.1 & 13.9 & 0.0 \\
\hline 6411.66 & FeI & 0.0 & 0.0 & 0.0 & 0.0 & 0.0 & 0.0 & 0.0 & 0.0 & 0.0 \\
\hline 6421.36 & FeI & 162.5 & 164.8 & 160.8 & 147.8 & 168.0 & 136.9 & 160.2 & 160.2 & 169.8 \\
\hline 6436.41 & FeI & 17.6 & 21.3 & 26.9 & 34.7 & 24.5 & 0.0 & 0.0 & 23.1 & 28.0 \\
\hline
\end{tabular}


P. Sestito et al.: Chemical abundances in open clusters. I., Online Material p 9

Table 10. continued.

\begin{tabular}{|c|c|c|c|c|c|c|c|c|c|c|}
\hline \multirow{2}{*}{$\begin{array}{l}\text { Wavelength } \\
(\AA)\end{array}$} & \multirow[t]{2}{*}{ Atom } & \multicolumn{9}{|l|}{$E W(\mathrm{~m} \AA)$} \\
\hline & & 17 & 18 & 19 & 25 & 27 & 45 & 938 & 940 & 941 \\
\hline 6481.88 & $\mathrm{FeI}$ & 101.9 & 105.8 & 109.3 & 107.9 & 104.5 & 96.4 & 103.3 & 106.8 & 111.4 \\
\hline 6498.94 & FeI & 102.2 & 102.0 & 97.7 & 108.6 & 103.3 & 88.3 & 95.7 & 103.5 & 108.6 \\
\hline 6518.37 & FeI & 86.6 & 85.6 & 87.1 & 87.2 & 87.3 & 74.4 & 87.9 & 87.3 & 88.8 \\
\hline 6533.94 & $\mathrm{FeI}$ & 45.5 & 52.4 & 53.0 & 53.4 & 63.2 & 47.9 & 59.0 & 50.7 & 57.1 \\
\hline 6574.25 & FeI & 82.3 & 80.7 & 82.4 & 87.6 & 89.3 & 60.9 & 79.1 & 78.4 & 89.6 \\
\hline 6581.22 & FeI & 66.3 & 68.8 & 75.7 & 79.8 & 74.7 & 54.1 & 61.1 & 64.1 & 70.8 \\
\hline 6591.31 & $\mathrm{FeI}$ & 14.8 & 20.7 & 16.7 & 25.9 & 19.7 & 16.3 & 0.0 & 17.1 & 21.7 \\
\hline 6593.88 & FeI & 121.8 & 125.5 & 121.9 & 133.3 & 129.5 & 107.9 & 119.9 & 125.9 & 127.9 \\
\hline 6608.04 & $\mathrm{FeI}$ & 53.4 & 51.4 & 54.8 & 53.6 & 51.9 & 37.0 & 45.7 & 47.2 & 51.2 \\
\hline 6609.12 & FeI & 105.1 & 106.7 & 105.7 & 114.2 & 113.8 & 96.8 & 103.9 & 105.6 & 106.1 \\
\hline 6625.04 & $\mathrm{FeI}$ & 62.9 & 63.2 & 63.9 & 70.4 & 77.9 & 52.8 & 62.5 & 66.5 & 70.7 \\
\hline 6627.56 & $\mathrm{FeI}$ & 37.1 & 40.5 & 41.1 & 45.1 & 43.3 & 33.7 & 0.0 & 37.4 & 42.2 \\
\hline 6633.76 & $\mathrm{FeI}$ & 77.8 & 83.3 & 77.5 & 84.2 & 88.0 & 65.7 & 80.9 & 80.0 & 80.5 \\
\hline 6667.43 & FeI & 26.0 & 23.8 & 30.7 & 29.6 & 30.3 & 12.7 & 0.0 & 18.4 & 25.7 \\
\hline 6667.72 & $\mathrm{FeI}$ & 18.5 & 24.8 & 16.3 & 0.0 & 22.1 & 0.0 & 0.0 & 0.0 & 19.3 \\
\hline 6699.14 & FeI & 16.4 & 14.5 & 8.5 & 0.0 & 20.5 & 13.1 & 0.0 & 13.5 & 19.0 \\
\hline 6703.58 & FeI & 65.0 & 71.3 & 62.9 & 73.9 & 74.9 & 56.3 & 73.7 & 65.2 & 71.4 \\
\hline 6704.48 & $\mathrm{FeI}$ & 12.9 & 10.5 & 13.6 & 16.0 & 14.7 & 9.1 & 0.0 & 10.9 & 11.6 \\
\hline 6713.74 & $\mathrm{FeI}$ & 26.4 & 28.3 & 29.6 & 32.8 & 39.4 & 0.0 & 30.2 & 27.5 & 30.3 \\
\hline 6725.36 & FeI & 30.0 & 30.2 & 29.5 & 43.0 & 34.1 & 15.8 & 35.1 & 26.8 & 33.5 \\
\hline 6726.67 & $\mathrm{FeI}$ & 57.9 & 61.8 & 60.4 & 63.2 & 61.2 & 53.1 & 0.0 & 52.9 & 59.9 \\
\hline 6733.15 & $\mathrm{FeI}$ & 41.0 & 37.3 & 39.6 & 50.1 & 43.7 & 28.7 & 38.1 & 36.3 & 35.7 \\
\hline 6739.52 & $\mathrm{FeI}$ & 44.2 & 46.9 & 38.5 & 48.4 & 56.4 & 31.2 & 46.2 & 39.8 & 47.2 \\
\hline 6745.97 & $\mathrm{FeI}$ & 0.0 & 0.0 & 14.5 & 0.0 & 28.2 & 0.0 & 0.0 & 0.0 & 0.0 \\
\hline 6750.16 & $\mathrm{FeI}$ & 112.0 & 117.0 & 112.3 & 122.4 & 116.6 & 108.7 & 110.0 & 118.0 & 118.4 \\
\hline 6753.47 & $\mathrm{FeI}$ & 17.1 & 16.7 & 13.3 & 0.0 & 0.0 & 0.0 & 0.0 & 0.0 & 0.0 \\
\hline 6756.55 & $\mathrm{FeI}$ & 0.0 & 12.0 & 0.0 & 0.0 & 11.0 & 14.9 & 0.0 & 0.0 & 0.0 \\
\hline 6786.86 & FeI & 41.3 & 43.9 & 40.4 & 46.5 & 46.4 & 34.7 & 35.3 & 41.5 & 41.1 \\
\hline 6793.26 & $\mathrm{FeI}$ & 31.9 & 16.3 & 22.7 & 30.8 & 22.5 & 20.6 & 23.2 & 0.0 & 32.4 \\
\hline 6796.12 & FeI & 27.0 & 32.8 & 24.9 & 37.6 & 26.0 & 21.1 & 26.3 & 29.1 & 0.0 \\
\hline 6804.30 & $\mathrm{FeI}$ & 21.9 & 0.0 & 0.0 & 38.2 & 31.8 & 0.0 & 29.6 & 31.4 & 0.0 \\
\hline 6806.86 & $\mathrm{FeI}$ & 67.6 & 0.0 & 0.0 & 79.2 & 76.8 & 75.5 & 63.7 & 71.6 & 0.0 \\
\hline 6810.27 & FeI & 67.1 & 0.0 & 0.0 & 64.7 & 67.3 & 58.8 & 59.0 & 61.4 & 0.0 \\
\hline 5525.14 & FeII & 35.8 & 27.3 & 28.7 & 32.2 & 0.0 & 0.0 & 28.4 & 32.9 & 33.7 \\
\hline 5534.85 & FeII & 86.4 & 81.4 & 82.2 & 72.0 & 78.7 & 70.6 & 75.2 & 75.9 & 84.2 \\
\hline 5627.50 & FeII & 19.8 & 0.0 & 13.3 & 21.3 & 18.7 & 10.1 & 20.0 & 17.7 & 22.5 \\
\hline 5991.38 & FeII & 52.3 & 47.2 & 46.2 & 37.8 & 45.4 & 48.5 & 51.2 & 54.2 & 50.9 \\
\hline 6084.10 & FeII & 32.1 & 34.5 & 33.9 & 32.5 & 35.9 & 0.0 & 39.8 & 35.8 & 35.2 \\
\hline 6113.33 & FeII & 28.3 & 29.7 & 25.1 & 19.2 & 18.5 & 0.0 & 23.1 & 19.9 & 23.1 \\
\hline 6149.25 & FeII & 47.2 & 45.3 & 53.1 & 54.9 & 42.2 & 36.1 & 42.7 & 47.9 & 43.8 \\
\hline 6239.95 & FeII & 0.0 & 0.0 & 29.4 & 0.0 & 0.0 & 0.0 & 0.0 & 0.0 & 30.9 \\
\hline 6247.56 & FeII & 63.9 & 64.5 & 63.0 & 52.4 & 53.1 & 53.2 & 66.2 & 66.4 & 67.0 \\
\hline 6369.46 & FeII & 30.6 & 32.6 & 32.8 & 27.9 & 27.2 & 21.0 & 31.4 & 35.2 & 29.1 \\
\hline 6383.72 & FeII & 0.0 & 0.0 & 0.0 & 0.0 & 13.0 & 0.0 & 0.0 & 16.9 & 0.0 \\
\hline 6416.93 & FeII & 46.1 & 51.9 & 48.1 & 41.4 & 47.0 & 39.4 & 50.6 & 55.6 & 53.0 \\
\hline 6432.68 & FeII & 56.9 & 59.7 & 59.5 & 58.3 & 48.7 & 50.8 & 58.4 & 57.3 & 59.6 \\
\hline 6456.39 & FelI & 84.8 & 84.7 & 84.7 & 71.3 & 75.4 & 62.1 & 88.2 & 92.7 & 84.4 \\
\hline 6516.08 & FeII & 79.8 & 73.0 & 73.2 & 65.6 & 64.6 & 49.3 & 67.7 & 76.6 & 72.1 \\
\hline
\end{tabular}


P. Sestito et al.: Chemical abundances in open clusters. I., Online Material p 10

Table 11. Equivalent widths for Fe lines in stars of NGC 2660.

\begin{tabular}{|c|c|c|c|c|c|c|}
\hline \multirow{2}{*}{$\begin{array}{l}\text { Wavelength } \\
(\AA)\end{array}$} & \multirow[t]{2}{*}{ Atom } & \multicolumn{5}{|l|}{$E W(\mathrm{~m} \AA)$} \\
\hline & & 296 & 318 & 542 & 694 & 862 \\
\hline 5494.47 & FeI & 54.3 & 50.7 & 49.5 & 49.6 & 45.8 \\
\hline 5521.28 & FeI & 0.0 & 15.7 & 0.0 & 0.0 & 0.0 \\
\hline 5522.45 & FeI & 66.8 & 68.8 & 70.0 & 69.6 & 71.3 \\
\hline 5524.24 & FeI & 0.0 & 0.0 & 0.0 & 0.0 & 0.0 \\
\hline 5539.29 & $\mathrm{FeI}$ & 29.3 & 39.2 & 41.2 & 36.2 & 44.6 \\
\hline 5547.00 & FeI & 49.1 & 53.3 & 57.2 & 57.0 & 52.6 \\
\hline 5552.69 & $\mathrm{FeI}$ & 6.9 & 14.6 & 15.7 & 12.5 & 0.0 \\
\hline 5560.22 & $\mathrm{FeI}$ & 69.1 & 74.5 & 67.3 & 57.2 & 73.9 \\
\hline 5568.86 & $\mathrm{FeI}$ & 29.3 & 28.4 & 33.7 & 24.6 & 28.7 \\
\hline 5577.03 & $\mathrm{FeI}$ & 0.0 & 0.0 & 0.0 & 0.0 & 0.0 \\
\hline 5586.77 & FeI & 220.1 & 207.0 & 210.7 & 206.0 & 194.4 \\
\hline 5587.58 & $\mathrm{FeI}$ & 61.8 & 63.8 & 63.0 & 47.6 & 62.0 \\
\hline 5595.05 & $\mathrm{FeI}$ & 14.2 & 12.0 & 15.9 & 0.0 & 0.0 \\
\hline 5608.98 & $\mathrm{FeI}$ & 25.5 & 23.5 & 22.0 & 21.5 & 17.2 \\
\hline 5609.97 & $\mathrm{FeI}$ & 16.4 & 18.8 & 16.2 & 0.0 & 0.0 \\
\hline 5611.36 & $\mathrm{FeI}$ & 17.8 & 25.0 & 23.1 & 21.7 & 21.0 \\
\hline 5618.64 & FeI & 68.2 & 69.4 & 73.8 & 73.9 & 71.4 \\
\hline 5619.61 & FeI & 51.3 & 59.4 & 54.9 & 56.0 & 52.4 \\
\hline 5635.83 & $\mathrm{FeI}$ & 49.6 & 51.4 & 55.9 & 62.1 & 59.9 \\
\hline 5636.70 & FeI & 47.8 & 35.0 & 46.6 & 44.4 & 47.0 \\
\hline 5650.00 & $\mathrm{FeI}$ & 57.7 & 51.3 & 50.2 & 54.9 & 56.0 \\
\hline 5651.48 & $\mathrm{FeI}$ & 32.6 & 38.7 & 33.7 & 29.1 & 25.9 \\
\hline 5652.33 & FeI & 48.9 & 50.4 & 45.4 & 49.6 & 44.3 \\
\hline 5661.02 & FeI & 11.8 & 0.0 & 0.0 & 0.0 & 0.0 \\
\hline 5661.35 & FeI & 42.3 & 45.5 & 49.2 & 44.6 & 50.6 \\
\hline 5677.69 & $\mathrm{FeI}$ & 10.2 & 14.2 & 13.0 & 13.2 & 15.6 \\
\hline 5678.39 & FeI & 8.8 & 0.0 & 15.2 & 0.0 & 12.3 \\
\hline 5680.24 & FeI & 23.3 & 23.1 & 31.3 & 22.3 & 23.8 \\
\hline 5701.56 & $\mathrm{FeI}$ & 108.5 & 118.9 & 120.9 & 119.5 & 120.0 \\
\hline 5717.84 & $\mathrm{FeI}$ & 85.5 & 88.6 & 92.5 & 89.3 & 80.3 \\
\hline 5731.77 & FeI & 73.6 & 78.8 & 84.9 & 80.8 & 74.3 \\
\hline 5738.24 & FeI & 29.8 & 21.7 & 27.7 & 27.9 & 22.1 \\
\hline 5741.86 & FeI & 60.9 & 60.0 & 59.6 & 55.4 & 54.6 \\
\hline 5742.96 & FeI & 21.9 & 22.5 & 25.7 & 25.0 & 20.1 \\
\hline 5752.04 & $\mathrm{FeI}$ & 75.5 & 73.9 & 75.7 & 75.1 & 64.8 \\
\hline 5754.41 & FeI & 52.1 & 34.4 & 0.0 & 40.8 & 0.0 \\
\hline 5759.26 & FeI & 6.9 & 14.2 & 16.4 & 0.0 & 19.2 \\
\hline 5760.36 & FeI & 45.8 & 50.0 & 0.0 & 43.6 & 49.5 \\
\hline 5775.09 & FeI & 0.0 & 0.0 & 0.0 & 0.0 & 0.0 \\
\hline 5778.46 & FeI & 0.0 & 0.0 & 0.0 & 0.0 & 0.0 \\
\hline 5784.67 & FeI & 0.0 & 0.0 & 0.0 & 0.0 & 0.0 \\
\hline 5793.92 & FeI & 0.0 & 0.0 & 0.0 & 0.0 & 0.0 \\
\hline 5806.73 & $\mathrm{FeI}$ & 0.0 & 0.0 & 0.0 & 0.0 & 0.0 \\
\hline 5811.91 & $\mathrm{FeI}$ & 0.0 & 0.0 & 0.0 & 0.0 & 0.0 \\
\hline 5814.81 & FeI & 0.0 & 0.0 & 0.0 & 0.0 & 0.0 \\
\hline 5835.11 & FeI & 26.3 & 20.8 & 0.0 & 36.6 & 41.9 \\
\hline 5837.70 & FeI & 9.9 & 21.9 & 23.6 & 0.0 & 0.0 \\
\hline 5849.69 & FeI & 0.0 & 17.1 & 33.8 & 0.0 & 24.6 \\
\hline 5852.23 & FeI & 50.3 & 61.2 & 64.7 & 67.8 & 58.3 \\
\hline 5853.15 & FeI & 23.5 & 33.6 & 37.8 & 35.0 & 24.8 \\
\hline 5855.09 & $\mathrm{FeI}$ & 23.2 & 34.9 & 38.5 & 32.1 & 39.7 \\
\hline 5856.10 & FeI & 52.8 & 58.3 & 53.7 & 60.2 & 52.7 \\
\hline 5858.78 & FeI & 20.5 & 28.4 & 30.5 & 28.7 & 16.7 \\
\hline 5859.60 & FeI & 84.7 & 96.4 & 98.5 & 95.8 & 93.6 \\
\hline 5861.11 & FeI & 17.5 & 19.0 & 14.3 & 0.0 & 0.0 \\
\hline 5862.37 & FeI & 98.2 & 104.3 & 98.0 & 108.5 & 103.4 \\
\hline 5879.49 & $\mathrm{FeI}$ & 26.1 & 33.6 & 29.0 & 0.0 & 32.0 \\
\hline 5880.02 & $\mathrm{FeI}$ & 0.0 & 17.9 & 17.4 & 25.6 & 21.2 \\
\hline 5881.28 & FeI & 27.1 & 33.3 & 25.6 & 31.7 & 35.7 \\
\hline 5902.48 & FeI & 24.6 & 0.0 & 30.5 & 24.6 & 22.1 \\
\hline 5905.68 & $\mathrm{FeI}$ & 75.3 & 78.3 & 73.2 & 79.7 & 67.0 \\
\hline 5927.80 & FeI & 56.0 & 59.5 & 63.1 & 60.5 & 60.5 \\
\hline
\end{tabular}


Table 11. continued.

\begin{tabular}{|c|c|c|c|c|c|c|}
\hline Wavelength & Atom & $E W(\mathrm{~m} \AA)$ & & & & \\
\hline$(\AA)$ & & 296 & 318 & 542 & 694 & 862 \\
\hline 5929.68 & FeI & 58.1 & 61.9 & 65.4 & 62.6 & 62.0 \\
\hline 5930.19 & $\mathrm{FeI}$ & 102.7 & 107.0 & 114.4 & 105.5 & 102.9 \\
\hline 5933.81 & $\mathrm{FeI}$ & 0.0 & 0.0 & 16.9 & 0.0 & 0.0 \\
\hline 5934.66 & $\mathrm{FeI}$ & 99.4 & 105.4 & 106.1 & 108.8 & 99.6 \\
\hline 5947.53 & $\mathrm{FeI}$ & 0.0 & 19.7 & 14.5 & 0.0 & 0.0 \\
\hline 5956.71 & FeI & 88.7 & 108.7 & 106.2 & 97.8 & 101.1 \\
\hline 5976.79 & FeI & 86.6 & 103.1 & 98.2 & 100.7 & 101.3 \\
\hline 5984.83 & FeI & 105.5 & 109.0 & 119.3 & 100.9 & 94.9 \\
\hline 6003.02 & $\mathrm{FeI}$ & 91.9 & 101.9 & 96.2 & 105.2 & 95.2 \\
\hline 6007.97 & $\mathrm{FeI}$ & 82.4 & 82.1 & 90.0 & 79.8 & 81.6 \\
\hline 6008.57 & $\mathrm{FeI}$ & 106.2 & 112.1 & 106.9 & 107.9 & 109.2 \\
\hline 6015.24 & FeI & 21.0 & 14.9 & 20.0 & 21.4 & 24.1 \\
\hline 6019.37 & FeI & 16.6 & 20.3 & 20.2 & 0.0 & 21.9 \\
\hline 6027.06 & FeI & 85.0 & 98.0 & 93.4 & 94.1 & 80.2 \\
\hline 6056.01 & FeI & 90.3 & 89.6 & 104.4 & 92.5 & 90.1 \\
\hline 6065.49 & $\mathrm{FeI}$ & 161.0 & 158.0 & 161.4 & 164.5 & 154.1 \\
\hline 6078.50 & FeI & 93.8 & 93.4 & 102.3 & 93.1 & 96.9 \\
\hline 6079.02 & $\mathrm{FeI}$ & 65.0 & 70.6 & 69.9 & 63.5 & 67.8 \\
\hline 6082.72 & $\mathrm{FeI}$ & 68.5 & 76.3 & 79.8 & 72.3 & 78.1 \\
\hline 6089.57 & FeI & 61.5 & 0.0 & 66.9 & 63.1 & 54.9 \\
\hline 6093.65 & FeI & 53.2 & 46.9 & 55.7 & 45.9 & 0.0 \\
\hline 6094.38 & FeI & 32.9 & 30.7 & 39.4 & 32.8 & 37.1 \\
\hline 6096.67 & $\mathrm{FeI}$ & 59.1 & 63.9 & 58.6 & 57.1 & 50.4 \\
\hline 6098.25 & $\mathrm{FeI}$ & 33.6 & 23.5 & 36.4 & 32.3 & 34.7 \\
\hline 6120.26 & $\mathrm{FeI}$ & 0.0 & 26.3 & 31.5 & 27.2 & 30.3 \\
\hline 6137.00 & FeI & 112.1 & 120.0 & 111.3 & 115.2 & 120.0 \\
\hline 6151.62 & FeI & 84.2 & 87.8 & 86.1 & 95.5 & 83.0 \\
\hline 6157.73 & FeI & 91.8 & 95.9 & 97.9 & 94.5 & 94.9 \\
\hline 6165.36 & $\mathrm{FeI}$ & 69.1 & 70.6 & 65.2 & 73.1 & 69.6 \\
\hline 6173.34 & FeI & 98.2 & 106.8 & 102.5 & 107.3 & 104.1 \\
\hline 6187.40 & $\mathrm{FeI}$ & 0.0 & 0.0 & 10.6 & 24.9 & 0.0 \\
\hline 6187.99 & $\mathrm{FeI}$ & 64.8 & 80.9 & 81.1 & 75.7 & 77.2 \\
\hline 6199.51 & FeI & 15.0 & 0.0 & 0.0 & 18.2 & 0.0 \\
\hline 6200.32 & FeI & 103.9 & 111.4 & 104.6 & 101.7 & 97.4 \\
\hline 6213.44 & $\mathrm{FeI}$ & 113.2 & 124.2 & 128.5 & 118.7 & 122.9 \\
\hline 6219.29 & FeI & 118.7 & 128.7 & 131.3 & 129.8 & 132.1 \\
\hline 6220.79 & $\mathrm{FeI}$ & 40.1 & 40.4 & 42.0 & 44.3 & 43.4 \\
\hline 6226.74 & $\mathrm{FeI}$ & 48.3 & 49.2 & 46.2 & 49.7 & 47.9 \\
\hline 6232.65 & FeI & 105.7 & 108.3 & 115.4 & 108.4 & 106.8 \\
\hline 6240.65 & FeI & 82.8 & 90.5 & 91.8 & 86.5 & 86.0 \\
\hline 6246.33 & FeI & 128.5 & 136.1 & 136.3 & 141.5 & 125.7 \\
\hline 6252.56 & FeI & 157.4 & 150.0 & 158.6 & 157.1 & 158.8 \\
\hline 6265.14 & $\mathrm{FeI}$ & 122.7 & 129.3 & 132.5 & 133.5 & 122.9 \\
\hline 6270.23 & $\mathrm{FeI}$ & 90.6 & 97.0 & 98.9 & 92.3 & 92.4 \\
\hline 6280.62 & FeI & 125.3 & 132.8 & 132.8 & 132.0 & 131.7 \\
\hline 6290.55 & $\mathrm{FeI}$ & 10.6 & 20.4 & 19.1 & 0.0 & 0.0 \\
\hline 6297.80 & FeI & 113.5 & 111.9 & 118.3 & 115.4 & 114.1 \\
\hline 6301.51 & FeI & 138.7 & 138.3 & 136.4 & 147.3 & 145.1 \\
\hline 6303.47 & FeI & 8.4 & 8.7 & 0.0 & 0.0 & 0.0 \\
\hline 6311.50 & $\mathrm{FeI}$ & 57.4 & 60.8 & 62.1 & 54.2 & 61.8 \\
\hline 6315.81 & FeI & 67.4 & 68.4 & 70.5 & 61.6 & 71.8 \\
\hline 6322.69 & $\mathrm{FeI}$ & 107.5 & 114.7 & 113.4 & 113.1 & 115.8 \\
\hline 6330.85 & $\mathrm{FeI}$ & 54.4 & 50.6 & 56.6 & 58.4 & 51.4 \\
\hline 6335.34 & FeI & 136.0 & 139.8 & 137.3 & 137.7 & 143.2 \\
\hline 6380.75 & FeI & 72.8 & 84.9 & 81.3 & 76.6 & 81.2 \\
\hline 6392.54 & FeI & 44.5 & 58.0 & 56.0 & 53.5 & 51.3 \\
\hline 6393.61 & $\mathrm{FeI}$ & 164.3 & 163.0 & 173.7 & 178.7 & 175.4 \\
\hline 6400.32 & FeI & 108.7 & 135.5 & 131.4 & 123.6 & 123.7 \\
\hline 6411.11 & FeI & 19.9 & 0.0 & 0.0 & 0.0 & 0.0 \\
\hline 6411.66 & FeI & 146.4 & 142.3 & 150.7 & 149.2 & 145.9 \\
\hline 6421.36 & $\mathrm{FeI}$ & 159.5 & 163.6 & 166.9 & 163.2 & 157.4 \\
\hline
\end{tabular}


P. Sestito et al.: Chemical abundances in open clusters. I., Online Material p 12

Table 11. continued.

\begin{tabular}{|c|c|c|c|c|c|c|}
\hline $\begin{array}{l}\text { Wavelength } \\
\text { (§) }\end{array}$ & Atom & $\begin{array}{l}E W(\mathrm{~m} \AA) \\
296\end{array}$ & 318 & 542 & 694 & 862 \\
\hline 6436.41 & FeI & 22.4 & 0.0 & 24.4 & 24.4 & 27.2 \\
\hline 6481.88 & $\mathrm{FeI}$ & 95.0 & 99.9 & 107.2 & 110.0 & 102.6 \\
\hline 6498.94 & $\mathrm{FeI}$ & 90.6 & 96.2 & 96.4 & 87.4 & 96.0 \\
\hline 6518.37 & $\mathrm{FeI}$ & 90.7 & 91.7 & 94.7 & 98.5 & 95.2 \\
\hline 6533.94 & $\mathrm{FeI}$ & 64.4 & 67.2 & 66.3 & 56.9 & 71.6 \\
\hline 6574.25 & FeI & 72.1 & 72.6 & 79.7 & 78.6 & 67.4 \\
\hline 6581.22 & FeI & 64.9 & 70.7 & 66.8 & 63.2 & 60.8 \\
\hline 6591.31 & FeI & 21.2 & 20.6 & 21.0 & 20.5 & 0.0 \\
\hline 6593.88 & FeI & 122.7 & 120.1 & 133.0 & 137.2 & 126.7 \\
\hline 6608.04 & $\mathrm{FeI}$ & 52.1 & 57.2 & 52.8 & 49.7 & 45.4 \\
\hline 6609.12 & $\mathrm{FeI}$ & 103.5 & 112.1 & 112.4 & 110.5 & 106.4 \\
\hline 6625.04 & $\mathrm{FeI}$ & 47.6 & 66.2 & 72.6 & 61.5 & 58.6 \\
\hline 6627.56 & FeI & 43.2 & 47.8 & 51.5 & 46.4 & 43.0 \\
\hline 6633.76 & FeI & 86.5 & 87.3 & 85.9 & 79.8 & 84.8 \\
\hline 6667.43 & FeI & 17.0 & 27.2 & 16.0 & 0.0 & 18.6 \\
\hline 6667.72 & FeI & 0.0 & 16.9 & 15.1 & 16.0 & 12.4 \\
\hline 6699.14 & $\mathrm{FeI}$ & 38.1 & 26.5 & 24.1 & 20.1 & 14.0 \\
\hline 6703.58 & $\mathrm{FeI}$ & 61.3 & 70.5 & 71.6 & 71.3 & 65.9 \\
\hline 6704.48 & $\mathrm{FeI}$ & 0.0 & 16.3 & 15.0 & 14.2 & 14.0 \\
\hline 6713.74 & FeI & 37.4 & 35.3 & 39.0 & 26.9 & 29.5 \\
\hline 6725.36 & FeI & 44.6 & 35.8 & 39.1 & 38.4 & 0.0 \\
\hline 6726.67 & FeI & 65.4 & 66.8 & 65.7 & 73.3 & 64.2 \\
\hline 6733.15 & $\mathrm{FeI}$ & 48.2 & 45.0 & 46.0 & 43.8 & 37.6 \\
\hline 6739.52 & $\mathrm{FeI}$ & 44.0 & 44.1 & 46.3 & 41.7 & 37.5 \\
\hline 6745.97 & $\mathrm{FeI}$ & 12.8 & 10.2 & 0.0 & 0.0 & 0.0 \\
\hline 6750.16 & FeI & 107.9 & 115.5 & 115.0 & 115.3 & 113.7 \\
\hline 6753.47 & FeI & 12.4 & 14.3 & 17.6 & 0.0 & 9.9 \\
\hline 6756.55 & FeI & 19.3 & 11.9 & 0.0 & 0.0 & 0.0 \\
\hline 6786.86 & FeI & 36.0 & 37.8 & 43.2 & 41.9 & 48.8 \\
\hline 6793.26 & FeI & 17.1 & 21.5 & 23.6 & 28.8 & 31.1 \\
\hline 6796.12 & $\mathrm{FeI}$ & 23.4 & 37.2 & 36.0 & 29.4 & 0.0 \\
\hline 6804.30 & $\mathrm{FeI}$ & 25.2 & 37.2 & 0.0 & 29.0 & 29.3 \\
\hline 6806.86 & FeI & 67.1 & 63.0 & 62.9 & 71.7 & 71.1 \\
\hline 6810.27 & FeI & 64.3 & 70.7 & 0.0 & 75.8 & 69.3 \\
\hline 5525.14 & FeII & 28.5 & 40.3 & 38.0 & 24.2 & 28.7 \\
\hline 5534.85 & FeII & 84.8 & 90.8 & 99.3 & 91.5 & 71.1 \\
\hline 5627.50 & FeII & 16.4 & 22.7 & 23.6 & 0.0 & 0.0 \\
\hline 5991.38 & FeII & 57.6 & 54.9 & 60.6 & 52.2 & 53.7 \\
\hline 6084.10 & FeII & 37.7 & 44.7 & 49.0 & 38.4 & 49.9 \\
\hline 6113.33 & FeII & 0.0 & 25.6 & 35.2 & 0.0 & 33.8 \\
\hline 6149.25 & FeII & 31.6 & 48.4 & 45.7 & 52.3 & 58.1 \\
\hline 6239.95 & FeII & 24.8 & 24.1 & 0.0 & 37.4 & 30.9 \\
\hline 6247.56 & FeII & 71.2 & 74.2 & 75.1 & 71.2 & 68.7 \\
\hline 6369.46 & FeII & 40.7 & 36.4 & 38.6 & 38.3 & 32.3 \\
\hline 6383.72 & FeII & 0.0 & 14.3 & 19.8 & 11.7 & 0.0 \\
\hline 6416.93 & FeII & 51.4 & 50.4 & 62.2 & 54.7 & 57.5 \\
\hline 6432.68 & FeII & 67.3 & 70.2 & 63.0 & 64.5 & 68.2 \\
\hline 6456.39 & FeII & 74.0 & 82.9 & 84.7 & 89.2 & 83.0 \\
\hline 6516.08 & FeII & 83.8 & 73.5 & 101.0 & 91.7 & 102.6 \\
\hline
\end{tabular}

